\title{
Resonance in Chirogenesis and Photochirogenesis: Colloidal Polymers Meet Chiral Optofluidics
}

\author{
Michiya Fujiki (D)
}

Citation: Fujiki, M. Resonance in Chirogenesis and Photochirogenesis: Colloidal Polymers Meet Chiral Optofluidics. Symmetry 2021, 13, 199. https://doi.org/10.3390/ sym13020199

Academic Editors: Rui Tamura and Ivan Fernandez-Corbaton

Received: 15 November 2020

Accepted: 18 January 2021

Published: 26 January 2021

Publisher's Note: MDPI stays neutral with regard to jurisdictional claims in published maps and institutional affiliations.

Copyright: (C) 2021 by the author. Licensee MDPI, Basel, Switzerland This article is an open access article distributed under the terms and conditions of the Creative Commons Attribution (CC BY) license (https:// creativecommons.org/licenses/by/ $4.0 /$ )
Division of Materials Science, Graduate School of Science and Technology, Nara Institute of Science and Technology, 8916-5 Takayama, Ikoma, Nara 630-0192, Japan; fujikim@ms.naist.jp

\begin{abstract}
Metastable colloids made of crystalline and/or non-crystalline matters render abilities of photonic resonators susceptible to chiral chemical and circularly polarized light sources. By assuming that $\mu \mathrm{m}$-size colloids and co-colloids consisting of $\pi$ - and/or $\sigma$-conjugated polymers dispersed into an optofluidic medium are artificial models of open-flow, non-equilibrium coacervates, we showcase experimentally resonance effects in chirogenesis and photochirogenesis, revealed by gigantic boosted chiroptical signals as circular dichroism (CD), optical rotation dispersion, circularly polarized luminescence (CPL), and CPL excitation (CPLE) spectral datasets. The resonance in chirogenesis occurs at very specific refractive indices (RIs) of the surrounding medium. The chirogenesis is susceptible to the nature of the optically active optofluidic medium. Moreover, upon an excitation-wavelengthdependent circularly polarized (CP) light source, a fully controlled absolute photochirogenesis, which includes all chiroptical generation, inversion, erase, switching, and short-/long-lived memories, is possible when the colloidal non-photochromic and photochromic polymers are dispersed in an achiral optofluidic medium with a tuned RI. The hand of the CP light source is not a determining factor for the product chirality. These results are associated with my experience concerning amphiphilic polymerizable colloids, in which, four decades ago, allowed proposing a perspective that colloids are connectable to light, polymers, helix, coacervates, and panspermia hypotheses, nuclear physics, biology, radioisotopes, homochirality question, first life, and cosmology.
\end{abstract}

Keywords: symmetry breaking; biomolecular handedness; circular dichroism; circularly polarized luminescence; non-equilibrium; colloid; absolute asymmetric synthesis; conjugated polymer; dissipative structure

\section{Introduction-Historical Backgrounds, Knowledge, Then, and Now \\ 1.1. What Is the Life?-Open System, Negative Entropy, Non-Equilibrium Thermodynamics}

Humankind has been thinking about life for a long time; where did life come from? Where will life go? Does life exist on earth only? So far, many scientists have postulated several scenarios throughout the primordial eons to address these curious questions. Regarding the first life on earth, the origin of biomolecular handedness remains an unanswered question in the scientific community [1-41].

In 1944, Erwin Schrödinger, one of the leading quantum physicists in his day, wrote an essay book titled What is Life? With Mind and Matter and Autobiographical Sketches [1]. Entropy inevitably increases only in a closed system-like universe as the consequence of the second law of thermodynamics. Living matters, thus, cannot elude conventional physics laws, but should involve other physics laws. To avoid a thermodynamic equilibrium by a spontaneous decay, living matters have to acquire and maintain negative entropy in an open system. Because living organisms exist as metastable states, metabolism that is a biological term of maintaining negative entropy is needed through all processes of eating, drinking, breathing, and, in case of plants, assimilating. To my knowledge, the two key concepts are rarely stated in most chemistry textbooks and not lectured in schools and colleges, although Schrödinger was the first to invoke an importance of these concepts. 
The chemistry textbooks and lectures teach us positive entropy, close system, enthalpydriven chemical reaction, and Arrhenius plot, but do not involve quantum tunneling, resonance, hierarchy from elemental particles, atoms, and molecules, four fundamental forces in nature, parity violation in weak nuclear force, the origin of homochirality, and the origin of life.

In 2002, Soffer et al. stated mathematically that non-equilibrium thermodynamics and equilibrium thermodynamics, respectively, correspond to the excited-state and the groundstate solutions of a Schrödinger wave equation [39]. In 2017, by referring to Schrödinger's notes, Ornes descried the core concept, concerning how non-equilibrium thermodynamics are connected to the mystery of life and other fields [40]. Non-equilibrium biological systems play key roles in gene transcription, diffusion in cytoplasm, transporting substances between cells with molecular motors, and cell signaling. Moreover, non-equilibrium phenomena are commonly recognized in the fields of fluid dynamics, plasma physics, meteorology, and astrophysics.

Likewise, an issue of left-right preference in the biomolecular handedness in the ground and excited states should involve these key concepts. When an equal probability of a simple chiral molecule, such as L- and D-alanine is considered, two possible states exist, corresponding to an increase in entropy. Living organisms only comprising optically active ingredients on Earth are possible to exist under open-flow conditions of energy and chemical resources because the metastable life requires low-entropy foods with chirality, and harvests solar/thermal energy $[1,10,17]$. If life existed on earth in the past, one of the greatest mysteries is whether stereogenic centers and/or stereogenic bonds in Hadean and Precambrian eons are identical to those of the on-going life on earth or are the extraterrestrial origin [22-38]. A conclusive answer to the question appears difficult because any fossil records of biomolecules and biopolymers associated with their handedness were decomposed. The snowball earth hypothesis, however, claims that prokaryotes inhabited the Precambrian eon for $\sim 2$ billion years $[27,28]$. Researchers in archaeological bacteriology have accepted that lighter ${ }^{12} \mathrm{C}$-containing stuffs are enriched in living organisms for their entire lifetimes. Indeed, the fact that ${ }^{13} \mathrm{C}$ in ${ }^{13} \mathrm{C}-/{ }^{12} \mathrm{C}$-isotopic ratio is significantly depleted would be evidence that the methanogenic microbes existed in the Archaean eon 3.5-3.8 billion years ago, associated with evolution of geodynamo of Earth, although Earth was born 4.54 billion years ago already [33-36].

Characterization of isotopic ratios and L-D ratios is powerful to discuss the origin of amino acids and carboxylic acids on Earth whether it is extraterrestrial origin or terrestrial origin. In 1987, Epstein et al. invoked that amino acids and carboxylic acids extracted from the Murchison meteorite were extraterrestrial in origin from isotopic ratios of $\mathrm{D}$ in $\mathrm{D} / \mathrm{H}$ and ${ }^{15} \mathrm{~N}$ in ${ }^{15} \mathrm{~N} /{ }^{14} \mathrm{~N}$ [37]. In 1997, Engel and Macko supported the extraterrestrial origin of amino acids and carboxylic acids in the meteorite from the analysis of the isotopic ratios and L-D ratios of alanine and glutamic acids [38].

Regarding the origin of biological homochirality associated with the first life on Earth, several scenarios are postulated. For example, the possible scenarios are classified to: (i) extraterrestrial and terrestrial origins; (ii) the by-chance and the necessity mechanisms; (iii) physical force origins and chemical substance origins. Importantly, all chemical processes and reactions should obey the physics laws regardless of extraterrestrial and terrestrial origins and chance-and-necessity mechanisms. The only exception would be that these physics laws, and chemistry, cannot apply to black holes.

\subsection{Physical Advantage Factors for Left-Right Asymmetry}

Goldanskii et al. comprehensively reviewed that 13 physical advantage factors called $g^{*}$ can cause a left-right preference to address the homochirality question at molecular and polymeric levels [42-44]. The $g^{*}$-values defined in physics may be a half of the Kuhn's anisotropy or Kuhn's dissymmetry ratio, $g$-values, defined in chemistry. The capabilities of chiral physical forces can access primitive molecules, followed by polymerization processes, which enable the homochiral world on terrestrial and extraterrestrial conditions. The global 
advantage factors are universal, deterministic for the biomolecular handedness, while the local advantage factors are mirror-symmetric, hence, provide the by-chance mechanism. Among the advantage factors, the $g^{*}$ of circularly polarized (CP) light source is considerably high on the order of $10^{-4}-10^{-2}$.

On the other hand, the $g^{*}$ for handed $\beta^{-}$-electron is extremely small, only $10^{-9}$ $10^{-11}$, while the values of $g^{*}$ for: (i) static magnetic field with linearly polarized light, known as the Faraday effect; (ii) Coriolis force (rotation) with static electric filed and static magnetic field; and (iii) Coriolis force with static magnetic field and gravitational field are on the order of $10^{-4}$. Coriolis force with gravitational force is a mirror symmetric chiral force, corresponding to the vectoral hydrodynamic flowing, often called swirling flow and vortex flow. Handed weak neutral current provides the smallest $g^{*}$ on the order of $10^{-17}$. Static magnetic field, static electric filed (so-called Stark effect), and gravitational field alone do not induce the left-right preference. CP-light, Coriolis force, static electric filed, static magnetic field, and gravitational field are parity-conserving mirror-symmetrical physical forces, while a weak neutral current and $\beta^{-}$-electron are parity-violating handed physical sources.

In 1953, Frank treated, mathematically, the first seminal bifurcation model account for a spontaneous mirror symmetry breaking in terms of evolution of biological homochirality [45]. For visibility and clarity, Goldanskii et al. illustrated two bifurcation models connecting to double-well and single-well potential curves [42-44]; a mirror-symmetric bifurcation model with a mirror-symmetric double-well potential curve and a non-mirrorsymmetric bifurcation model with a non-mirror-symmetric double-well potential curve. Based on these bifurcation models associated with the $g^{*}$ values discussed above, Goldanskii et al. showed two scenarios, allowing us to well recognize both the bi-chance and necessity mechanisms for the spontaneous mirror symmetry breaking. Knowing the fluctuation behaviors around these bi-furcation points with those $g^{*}$ values, namely, a phase transition characteristics from the single-well to the double-well or from the double-well to the single-well is the key to rationally design the left-right preference, followed by the homochirality systems $[7,8,46]$.

\subsubsection{Circularly Polarized Light Source}

Historically, in 1874, LeBel, and in 1894, van't Hoff, proposed independently a possibility of absolute asymmetric synthesis (AAS) using $r$ - and l-CP light as chiral physical source [46-49]. A half century after their predictions, in 1929, Kuhn and Broun experimentally realized their conjectures as photodestruction mode AAS [46]. Their pioneering work prompted allowed many researchers to investigate AAS for a century because expensive chiral chemical substances are no longer needed [47-50]. However, most researchers have long believed that $l$-CP light produces left-hand (or right-hand) molecules preferentially and vice versa because the product chirality is determined solely by the hand of $\mathrm{CP}$ light. The AAC with $r$ - and $l$-CP light covers absolute asymmetric photosynthesis, photodestruction, and photoresolution modes [47-49]. Generating left-right preference endowed with CP-light was coined photochirogenesis by Yoshihisa Inoue as the key concept of CP light-matter interactions in 1996 [7,8,51].

In the 1970s, Calvin et al. reported an anomaly of an excitation wavelength dependent CP light-driven photosynthesis mode AAS recognized as a switching product chirality of [8]-helicene in homogeneous toluene solution [52]. In 2014, Meinert et al. reported the wavelength-dependent CP light-driven photodestruction mode AAS revealing switching chirality when rac-alanine film was decomposed upon irradiation of two vacuum-UV light sources (184 and $200 \mathrm{~nm}$ ) [53]. A recent development of wavelength-dependent CP light driven photoresolution mode AAS employed by us will be given in Section 4.5 [54-56].

\subsubsection{Static Electric Field}

According to recent works, with static electric fields, so-called Stark effects, in the absence of magnetic field, Auzinsh et al. reported chirogenesis as emission modes on 
the order of $g=0.20\left(10 \%\right.$ for circularity) at ${ }^{7} D_{3 / 2}$ state and $g=0.15$ (7.5\% for circularity) at ${ }^{9} D_{3 / 2}$ state of parity-violating, paramagnetic Cs vapor placed between two mirrored electrodes [57]. On the other hand, Datta et al. indicated an occurrence of lowering molecular symmetry of achiral diamagnetic aromatic molecules by a computer simulation. Coronene, a 6-fold highly symmetric molecule, undergoes $D_{6 \mathrm{~h}} \rightarrow C_{2}$ distortions via vibrational instability upon application of sufficiently intense static external electric field [58]. The electric field induced structural distortion is understood as resulting from excess charge accumulation of planar rings circumvented by symmetry lowering. Contrarily, tribenzopyrene, a contorted polyaromatic compound due to a repulsion by two pairs of syn $\mathrm{H}$-atoms, undergoes, spontaneously, $C_{2 \mathrm{v}} \rightarrow C_{2}$ distortions in the absence of the electric field. Coronene, tribenzopyrene, and other fused aromatics are assumed to be the representative polycyclic aromatic hydrocarbons, so-called PAHs, which are abundant in the universe [59]. Because $C_{2}$-symmetric molecules are chiral, a naive question remains to be elucidated experimentally, whether $C_{2}$-coronene and $C_{2}$-tribenzopyrene exist as racemic mixtures or single enantiomers in connection with the homochirality question in the universe. Collision-free, gas-phase circularly polarized luminescence (CPL) spectroscopy may allow us to provide an answer to this question.

\subsubsection{Static Magnetic Field}

With the origin of biological chirality in mind, Pasteur was the first to attempt a chemical process called enantioselection, showing whether left-handed or right-handed molecules are produced under magnetic field, but failed because the magnetic field is a pseudo-vector that cannot be coupled with molecular chirality [60]. In 1994, Zadel et al. published an astonishing paper titled Enantioselective Reactions in a Static Magnetic Field [61]. Several research teams attempted to reproduce these results immediately, but failed [62,63], because, prior to the experiments, in the solutions, under 2.1 Tesla of static magnetic field (NMR instrument) in the paper, all reaction solutions were in advance design and manipulated [64]. Static magnetic field on the order of 2 Tesla does not induce any detectable enantioselective reaction in a fluidic solution under non-restricted Brownian molecular motions. Uniform static magnetic fields do not induce molecular chirality because of the time-odd, axial vector $[60,65]$

Naaman and Wadeck reviewed theory and experiments of chiral-induced spin selectivity (CISS), where ordered films of chiral molecules on surfaces can act as electron spin filters [66]. By applying the idea of CISS, in 2018 and 2019, Naaman and his international team succeeded in enantiospecific crystallization of three L-/D-amino acids and thiolated L-/D-alanine-based helical oligomers at the magnetized surface with a gold-coated ferromagnet cobalt film $[67,68]$. Enantioselectivity is determined by north-up and south-up geometry of the magnet and the nature of chiral substances. An attractive force between electrons in the substrate and in the molecules is on the order of several tens of $\mathrm{kJ} \mathrm{mol}^{-1}$ when a molecule surface distance is $0.1-0.2 \mathrm{~nm}$ [68]. Brownian motion of floppy molecules is considerably restricted at the molecule surface interface, followed by a great suppression of the molecular motions during crystallization at the surface. Since a magnetic field is a short-distance force compared to the electric field, a proximity effect of the molecule at the magnetized surface should be considered.

In 1955, Akabori proposed the polyglycine hypothesis-foreproteine as the origin of protein homochirality on Earth [22]. The hypothesis involves three steps: (i) formation of aminoacetonitrile from formaldehyde, ammonia, and hydrogen cyanide, which were ubiquitous in primordial Earth; (ii) polymerization of aminoacetonitrile at a solid surface, such as a Kaolinite that is a two-dimensional aluminosilicate $\mathrm{Al}_{4} \mathrm{Si}_{4} \mathrm{O}_{4}(\mathrm{OH})_{4}$, followed by hydrolysis to yield polyglycine and ammonia; (iii) introduction of side chains to polyglycine. The experimental results showed that: (i) methylene groups of polyglycine are adsorbed on Kaolinite; and (ii) glycyl residues are converted to serine and threonine residues reacted with formaldehyde and acetaldehyde, respectively. Although achiral glycine has two equal $\mathrm{C}-\mathrm{H}$ bonds, the two $\mathrm{C}-\mathrm{H}$ bonds at each residue of helical oligoglycine and polyglycine 
are no longer equal. The two $\mathrm{C}-\mathrm{H}$ bonds at each glycine residue of the helix may reveal different reactivity toward chemical species. Akabori conjectured that the origin of all L-selectivity in amino acids of proteins results from the by-chance mechanism because there is no chirality in Kaolinite. The hypothesis, followed by experiments, were reported just before the groundbreaking fever in 1956-1958 that the parity in the $\beta$-decay process of ${ }^{60} \mathrm{Co}$ is violated, though a left-right equality of enantiomers was a concrete common sense in chemistry in those days [69-76].

Knowing the magnetic minerals and the first geodynamo on Earth is an important clue how Earth's core, atmosphere, biomolecular chirality, and life evolved [33-36]. In 2015, Tarduno et al. claimed that, by analyzing inside of zircon crystals, which were indestructible for nearly 4.4 billion years, in Western Australia, Earth's magnetic field had evolved already from more than 4 billion years ago [33]. Likewise, from the oldest rocks in South Africa, Earth's magnetic evolved around 3.5 billion years ago [34]. Moreover, a recent work indicates that, from magnetic minerals in ancient Greenlandic rocks, Earth's magnetic field arose at least 3.7 billion years ago [35,36], enabling to magnetically shield primitive life and chiral substances evolved on Earth.

The CISS theory [66], along with the experimental results $[67,68]$ proven by Naaman et al., encourages to test whether: (i) helical oligoglycine and polyglycine are adsorbed vertically at the magnetized surface; (ii) the two $\mathrm{C}$-H bonds at each residue reveal a different chemical reactivity toward several chemical species in the absence and presence of UV-light around 190-220 nm; and (iii) enantioselectivity is determined solely by north-up and south-up geometry.

In 2019, Stevenson and Davis proposed a possibility of magnetophoresis that radical species of D- and L-substances are separable and sortable under an ultrastrong magnetic field gradient of $>4.4 \times 10^{9}$ Tesla [77]. Such an ultrastrong magnetic field and gradient can no longer obey the standard Maxwell equations. Paramagnetic chiral molecular species in the universe may be passing through in the vicinity of magnetar, which are newborn neutron stars spinning very fast that generate the ultrastrong magnetic field. Kouveliotou et al. think that more than 100 million magnetars are wandering through the interstellar universe and trillions of organic paramagnetic materials are imbedded in cold molecular clouds [78]. The ultrastrong magnetic field causes vacuum birefringence, photon splitting, scattering suppression, and distortion of atoms [78]. Although magnetic field gradients are widely utilized in NMR imaging, so-called MRI, the feasibility of the ultrastrong magnetic field gradient is challenging.

\subsubsection{Hydrodynamic Flowing as a Model of Coriolis Force with Gravitational Force}

When artificial molecular chromophores and luminophores are dispersed in the fluidic medium, hydrodynamic swirling flowing clockwise (CW) and counterclockwise (CCW) are experimentally testable models to validate the origin of the gravitational field's left-right preference [79-83]. The vectoral hydrodynamic flowing is often called as swirling flow and/or vortex flow. The unidirectional hydrodynamic flowing in the CW or CCW direction imparts the left-right preference at the molecular and supramolecular levels. The left-right preference in the northern hemisphere may be opposite to that in the southern hemisphere due to parity-conserving physical force on Earth.

In 1993, Ohno et al. reported chirogenesis of circular dichroism (CD)-active J-aggregates from achiral free-base porphyrin derivative in acidic water solution by mechanically swirling in CW and CCW directions [79]. They observed two clear couplet-like CD bands around 410-450 $\mathrm{nm}$ due to Soret band and 480-500 nm due to Q-band. The couplet-like CD band profiles were inverted by choosing the $\mathrm{CW}$ or $\mathrm{CCW}$ direction during the propagation of J-aggregate. Very weak non-couplet CD band around $480 \mathrm{~nm}$, however, was recognized under a stagnant condition. Obviously, the hydrodynamic flowing appears responsible for the chirogenesis, but a statistical analysis was not performed yet. It is unclear whether this event occurs using the specific porphyrin derivative at a specific laboratory. 
In 2001, Ribó et al. confirmed, by the statistical analysis from nearly a hundred of independent experiments that macroscopic swirling forces in the $\mathrm{CW}$ and CCW directions indeed generate homochiral aggregates from water soluble achiral porphyrin derivatives that slightly differ from the porphyrin above [80]. The hydrodynamic flowing indeed acted as a trigger of the spontaneous homochiral $l$-or- $r$ aggregation [81].

Although water-soluble porphyrin derivatives carrying multiple phenyl rings at mesopositions are directly connected to biomolecular substances, the idea of hydrodynamic flowing is applicable to chain-like synthetic polymers and several chromophore/luminophore during their association processes that feel the handed force of the hydrodynamic flowing, regardless of the northern and the southern hemispheres on Earth and other exoplanets. The author assumes that, since unsubstituted free-base porphyrin framework is very floppy, these porphyrin derivatives substituted with tetraaryl groups at meso-positions may adopt dynamically twisting two structures, between left and right, in a fluidic solution at ambient temperature.

In line with the bifurcation scenario, in 2011, Okano et al. succeeded in chirogenesis from achiral rhodamine $B$ as a red luminescent molecular probe doped to a water-soluble sol-gel polymer, revealed by CPL spectroscopy [82]. Based on a statistic analysis, the sign in CPL signals at $580 \mathrm{~nm}$ was controlled by the CW/CCW motions of hydrodynamic flowing in a cuvette. The critical temperature $\left(T_{\mathrm{C}}\right)$ for gelation was controlled by tuning the concentration of the polymer in aqueous solution. The polymer solution was in a sol state with a low viscosity, while stirring above the $T_{\mathrm{C}}$, while the solution below the $T_{\mathrm{C}}$ spontaneously underwent a non-flowing gel state in a very high viscosity.

In 2018, by mimicking submarine rock micropores at primordial Earth, Liu and collaborators designed a sophisticated microfluidic chirogenesis system [83]. The fluidic system was composed of ten pairs of inclined microchambers to efficiently generate pairs of $\mathrm{CW}$ and CCW microvortices using achiral 3-fold molecular symmetric aromatic molecules that possessed supramolecular gelation capability. By a statistical analysis based on 56 totally independent experiments, they ascertained that the microfluidic chirogenesis system works very efficiently by simulating a behavior of hydrodynamic flowing in the microchamber. They re-confirmed that the microfluidic system is viable for a very rapid chirogenesis within $1 \mathrm{~ms}$ of the water-soluble porphyrin derivative. These results should shed light on the origin of the biomolecular homochirality; how oceanic vortices play a critical role in protein folding and self-organization in primordial Earth.

\subsubsection{Static Magnetic Field with Polarized Light}

Static magnetic field with linearly polarized light and static magnetic field with unpolarized light, which are called magneto-optical effects, including Faraday and polar Kerr effects, are possible to be chiral physical sources, enable to induce mirror symmetrical, left-right imbalance in the ground and photoexcited states. The left-right chiroptical preference from achiral substances in the ground and photoexcited states is determined by north-up and south-up magnetic fields to propagation of incident light, called magnetic circular dichroism (MCD) [84-87] and magnetic circularly polarized luminescence (MCPL) [88-91], respectively. The phenomena are closely connected to Zeeman splitting of degenerate molecular orbitals. Recently, Imai, Fujiki, and coworkers verified mirror symmetrical MCPL characteristics from diamagnetic achiral organic and racemic lanthanide luminophores. These luminophores radiate $l$ - and $r-\mathrm{CP}$ light upon unpolarized light at north-up and south-up Faraday geometry [92-94]. However, it is obscure whether mirror symmetrical MCPL arises from Zeeman splitting of degenerate molecular orbitals in the ground states or in the photoexcited states because a comparison with the corresponding MCD spectra is not elucidated yet. Nevertheless, in analogy with the first report of Raman scattering in 1928 [95], the MCPL characteristics teach that the handed light emission under external magnetic field is becoming a new type of secondary radiation sources, $l$ - and $r$-CP light, in the absence of any chemical chirality, when achiral luminophores are, by-chance, placed on magnetized substances (even on ubiquitous), but a weak geomagnetic field of 
$\sim 3 \times 10^{-5} \mathrm{~T}$. In that case, the magnetic fields should satisfy north-up and south-up Faraday geometries. A magnetic field-caused CP light source from achiral luminophores may be ubiquitous in the universe, and was therefore the source of circularly polarized radiation as seeds of molecular chirality in the past (and now all over universe).

Rikken and Raupach designed a static magnetic field-driven photoresolution molecular system using racemic $\mathrm{K}_{3} \mathrm{Cr}^{\mathrm{III}}$ (oxalate) $)_{3}$ complex in water upon irradiation of Ti:sapphire laser at $696 \mathrm{~nm}$ [96]. They demonstrated an occurrence of mirror-symmetrical photoresolution from the complex, in which a preferential chirality is determined by north-up and south-up Faraday geometries. Recently, Sharma calculated (based on the MCD effect) that unpolarized ultraviolet sunlight, so-called UV-C region, combined with atmospheric paramagnetic oxygen diluted with carbon dioxide can possibly trigger an initial enantiomeric excess (ee) to determine the biomolecular handedness under the geomagnetic force in Archaean Earth [97]. Notably, when the UV-C light destructs several biomolecular constituents, a preferential chirality between purine and pyrimidine nucleosides depends on a partial pressure of carbon dioxide. However, a preference in the product chirality of amino acids is always L-form. Thus, a static magnetic field with unpolarized light becomes one candidate for efficient photodestruction, and photoresolution modes in racemic amino acids and nucleosides in water disregard of terrestrial and interstellar conditions.

\subsubsection{Longitudinally Polarized Left-Handed $\beta$-Electron and Right-Handed $\beta$-Positron}

In 1956, Lee and Yang theoretically indicated a possibility of parity-violation in certain nuclear reactions. In 1957, Wu et al. experimentally confirmed the parity-violation in the reaction ${ }^{60} \mathrm{Co} \rightarrow{ }^{60} \mathrm{Ni}+e^{-}+$anti- $v_{\mathrm{e}}$ using $\beta^{-}$-decay of radioisotope ${ }^{60} \mathrm{Co}$. Likewise, a parityviolation in $\beta^{+}$-decay process that the ${ }^{58} \mathrm{Co} \rightarrow{ }^{58} \mathrm{Fe}+e^{+}+v_{\mathrm{e}}$ was confirmed, while positron and anti- $v_{\mathrm{e}}$ were antimatters of electron and $v_{\mathrm{e}}$, respectively [69-76]. The parity-violation relies on a weak charged current of the radioisotopes. In 1958, Goldhaber et al. determined that helicity of massless electron neutrino is left-handed from the resonance scattering experiment of $\gamma$-ray at $960 \mathrm{keV}$ that an excited nucleus ${ }^{63} \mathrm{Sm}^{152}$ (1- state) spontaneously radiates a handed $\gamma$-ray to relax a ground state nucleus ${ }^{62} \mathrm{Sm}^{152}$ (0+ state) [74].

In 1959, this discovery prompted Vester and Ulbricht to propose so-called VesterUlbricht (V-U) hypothesis: parity-violating, spin-polarized left-handed $\beta$-electron causes circularly polarized bremsstrahlung that preferentially decomposes D-amino acids and left-handed DNA precursors, thus remaining $l$-amino acids and right-handed DNA on Earth $[98,99]$. In 1984, Bonner reported the failure of the V-U hypothesis-that several racemic amino acids cause radioracemization when polarized $\beta$-electron Bremsstrahlen from radioisotopes ${ }^{90} \mathrm{Sr}-{ }^{90} \mathrm{Y},{ }^{14} \mathrm{C}$, and ${ }^{32} \mathrm{P}$ is applied [100].

In 1982 and 1984, Zitzewitz et al. utilized low-energy, right-handed $\beta$-positron in place of left-handed $\beta$-electron, and reported a weak asymmetry of leucine while cystine and tryptophan had little V-U effects [101,102]. In 2014, Dreiling and Gay confirmed successfully that the $\mathrm{V}-\mathrm{U}$ hypothesis is valid, based on the detection of a preferential L-D decomposition of racemic 3-bromocamphor [103]. In 2018, Dreiling et al. confirmed volatile ester derivatives of DL-amino acids and DL-sugar molecules with low-energy $\beta$-electron and $\beta$-positron may be suited to verify the $\mathrm{V}-\mathrm{U}$ hypothesis.

\subsubsection{Handed Weak Neutral Current Mediated by $Z^{0}$ Boson}

The $\beta^{-}$- and $\beta^{+}$-decay processes are mediated by massive $\mathrm{W}^{-}$boson $(80.4 \mathrm{Gev})$ and $\mathrm{W}^{+}(80.4 \mathrm{Gev})$ boson, respectively. On the other hand, parity-violating weak neutral current mediated by massive $Z^{0}$ boson $(91.2 \mathrm{Gev})$ is ultimately a handed chiral physical bias for subatoms, atoms, molecules, supramolecules, polymers, colloids, crystals, inorganics, possibly our life, and upward disregard of terrestrial and extraterrestrial origins. According to the electroweak theory, massive three $\mathrm{W}^{-}, \mathrm{W}^{+}, \mathrm{Z}^{0}$ bosons and massless photon (boson, light) can be unified to the same family above $\sim 10^{15} \mathrm{eV}$ [104]. Upon rapid cooling of our universe, the parity-violating weak force and parity-conserving electromagnetic force are separated at $\sim 10^{15} \mathrm{eV}$ by the bifurcation mechanism. 
Since the discovery in 1956-1957 of the parity-violating $\beta^{-}$- and $\beta^{+}$-decay processes [69-76], people assumed that the handed weak neutral current is responsible for deterministic factor of L-amino acid and D-ribose [3,6,105]. Parity-violation hypothesis in atoms, so-called atomic parity-violation, is established theoretically and experimentally, based on absorption and photoluminescence spectroscopy of collision-free atomic vapors [106-112]. As for the question: "Is parity violated in atoms?"-most atomic, elemental particle, and cosmological physicists say, no question, it is common sense. On the other hand, to the similar question of "Is parity violated in close-shell chiral molecules?" — most chemists believe it is skeptical, no question, nonsense, a big burden in one's research carrier and honor.

Up until now, many scientists in chemistry and molecular physics argued theoretically a possibility of parity-violation in molecules, in relation to the origin of biomolecular handedness on Earth, often called molecular parity-violation hypothesis. Parity-violation energy difference (PVED) of L- and D-molecules predicted theoretically is ultrasmall on the order of $10^{-8}-10^{-14} \mathrm{kcal} \mathrm{mol}^{-1}$ or $10^{-9}-10^{-15 \%}$ ee. In 1983, Mason and Tranter showed that, from calculation of the PVED with Slater-type-orbitals (STO) with 6-31G basis set, L-alanine and L-polypeptide in $\alpha$-helix and $\beta$-sheet are energetically stabilized compared to the corresponding D-alanine and D-polypeptide, due to parity violating weak nuclear current $[3,113]$. However, the PVED is ultrasmall on the order of $10^{-23} \mathrm{kcal} \mathrm{mol}^{-1}$. Most chemists, therefore, think that the molecular parity-violation hypothesis is doubtful and skeptical. Even if the hypothesis is true, it is experimentally impossible to prove by ordinary spectroscopy and enantioseparation column chromatography.

Most theories are treating ideal isolated molecules, including simple amino acids, carbohydrates, hypothetical, and realistic molecules, including heavier atoms in a vacuum at absolute temperature of zero-Kelvin. Additionally, the molecular parity-violation theorists always require experimentalists, who directly detect the tiny PVED for a pair of left- and right-molecules and well-defined oligomers in a collision-free condition, such as a reduced pressure, using precision high-resolution spectroscopy. Theorists think that solutions, solids, aggregates, gels, and polymers should be excluded in testing the molecular parityviolation hypothesis. Most theories focused on the PVED at the electronic, vibrational, and ro-vibrational ground states [3,113-117]. Experimentalists attempted to detect the PVED as absorption mode, using several sophisticated high-resolution spectrometers [118]. In 2003, Berger calculated the PVED of formyl aldehyde, $\mathrm{HFC}=\mathrm{O}$, in the electronically excited states that are suited to experimentally test the PVED hypothesis because of an enhanced PVED amplitude from a well-defined photoexcited parity [119].

In an analogy of Kasha's rule in photochemistry, radioisotopes ${ }^{60} \mathrm{Co}$ and ${ }^{58} \mathrm{Co}$ as excited states of the corresponding stable isotopes ${ }^{60} \mathrm{Ni}$ and ${ }^{58} \mathrm{Fe}$, respectively, allowed to unveil the parity-violation in their $\beta^{ \pm}$-decay radiation processes. Similarly, detecting atomic parity violation as emission mode from atomic vapors is more obvious, rather than absorption mode [109-112]. These spontaneous radiation processes to the ground state, connecting to parity, commonly arise from the far-from, non-equilibrium excited state.

The $\beta^{ \pm}$-decay process, atomic parity violation as emission mode, and Berger's theory stimulated the author and coworkers to measure spontaneous radiation processes from $\mathrm{S}_{1}$ state of nearly sixty non-rigid rotamers as achiral and/or racemic luminophores homogeneously dissolved in achiral solvents (not optofluidic solvents) in the photoexcited states to detect difference as emission modes between L-D molecular state upon excitation of unpolarized light $[120,121]$. All of these rotamers showed (-) sign CPL signals in the UV-visible region, suggesting temporal generation of energetically inequal, non-racemic structures under conditions of far-from, open-flow, non-equilibrium $S_{1}$-state, realized only upon a continuous irradiation of incoherent unpolarized light. Unpolarized light is an equal mixture of left- and right-CP light. For comparison, unsubstituted achiral fused aromatic luminophores that adopt rigid planar structures did not show CPL signals. Furthermore, D- and L-camphor, an enantiomeric pair of rigid chiral XYC $=\mathrm{O}$ dialkyl ketone luminophores, showed ideal mirror-image CPL spectra. From these comprehensive 
results, the author and coworkers invoked that the parity of non-rigid molecular rotamers in the photoexcited is violated, followed by radiating $(-)$ sign CPL signals without any exception $[120,121]$.

Currently, experimentally testing the molecular parity-violation hypothesis remains a hot topic and very challenging. Collision-free, gas-phase CPL spectroscopy may be a useful tool to provide a definitive answer to this long-standing question. Although this topic is not a major issue in this paper, the author, his coworkers, his students, his researchers, and his technical staff are aware of the facts that non-ideal mirror-image CPL and CD spectral characteristics can be often seen in our colloidal polymers dispersed in optofluidic media with tuned RIs. Some of the readers may be aware of the non-ideal mirror-image CPL and CD spectral characteristics in this paper. The author conjectures that these non-ideal mirror-image chiroptical characteristics from the colloidal polymers in tuned optofluidic media could be connected to certain mechanisms, in an enhancement in weak neutral currents at molecular and polymer levels. Those who are interested in this topic should read related parity-related reviews, monographs, and original papers, shown above.

\subsection{Extraterrestrial Origins of Life and Chirality_Panspermia Hypotheses}

Apart from the physical aspects in the previous sections, the fascinating, awesome extraterrestrial scenarios might be several panspermia hypotheses [122,123]. Panspermia means seeds everywhere in a Greek word. The panspermia hypothesis assumes that seeds of life ubiquitously exist in the past and, even now, all over universe, allowing to spread from one to another spaces and locations. The hypotheses may invoke us that seeds of chirality are ubiquitous in the past and, even now, all over universe.

In the late 19th century, Louis Pasteur, William Thomson (Lord Kelvin), and Hermann von Helmholtz conjectured extraterrestrial origin of life (and biological chirality). In 1903, Svante Arrhenius, Swedish physicist and chemist, proposed the radio-panspermia hypothesis; certain seeds of life with sizes of 200-300 nm traveled by radiative pressure of solar light and stellar light, landed on Earth [122]. In the 1960s-1980s, Hoyle and Wickramasinghe hypothesized cometary panspermia based on detection of astronomical radiation and reflectance in the range of $2.5-4.0 \mu \mathrm{m}\left(\sim 3000-3800 \mathrm{~cm}^{-1}\right)$ from dust coma of Comet Halley and Comet 67P/Churyumov-Gerasimenko [123]. The cometary panspermia, including comets, interstellar dusts, and asteroids, can carry several viruses and microbials, which landed on Earth from the primordial era (and even now) [123]. The scenarios led to propose further lithopanspermia (often referred to interstellar panspermia) and ballistic panspermia hypotheses (often referred to interplanetary panspermia). In lithopanspermia, impact-expelled rocks and meteorites from a surface of planet acts as spaceships to spread biological materials and microbials from one solar system to another solar system, while, in ballistic panspermia, the same events occur within the same solar system.

In 2013, Kawaguchi, Yamagishi, and coworkers hypothesized massapanspermia that microbial aggregates are transferrable between planets [124]. In 2020, they confirmed that aggregates of Deinococcus radiodurans (a representative radioresistant bacteria) can survive under the very severe space environment for 2-8 years, enabling the interplanetary travel that delivers the seeds of life and related chemical stuffs for many years [125]. The new hypotheses might encourage astrobiologists and astrochemists to pursue the possibility of the extraterrestrial life and related chemical substances, including liquid water and chiral molecules conducted by sophisticated spacecrafts, orbiting telescopes, and high-resolution spectrograph on Earth, followed by recent triumphs in astroscience.

For example, astroscientists confirmed plumes of water vapor and are now confident about the existence of water ocean on Europa (that is one of Jupiter's moons), with help from auroral emissions of hydrogen and oxygen by the Hubble space telescope (HST, National Aeronautics and Space Administration: NASA), observation by the Galileo spacecraft (NASA), and direct detection of water vapor using near-IR spectrograph at the 10-m Keck Observatory (Hawaii) [126]. The Rosetta space probe (European Space Agency: ESA) detects prebiotic constituents and water on Comet 67P/Churyumov-Gerasimenko, one 
of the comets in the Jupiter family $[127,128]$. The Cassini spacecraft (NASA), the Saturn probe launched in 1997, discovers water under the surface of Enkelados, which is one of the Saturnian moons, with help from the quadrupole gravity measurement system [129]. JPL (Jet Propulsion Laboratory at NASA) reported that Titan, which is the largest Saturnian moon, possesses water comprising inorganic salts [130]. The Kepler space telescope (NASA) discovered over 2600 exoplanets; among them, numbers of Earth-like planets are hidden in habitable zones [131,132]. In 2016, astronomers discovered interstellar chiral propylene oxide generating in SagittariusB2 of the Milky Way Galaxy by analyzing the unique absorption bands in the range of mm-wavelength of Parkes radio telescope at Australia Observatory [133]. A preference between L- and D-propylene oxides remains obscure because the telescope is not a polarized microwave spectrometer. In 2019, an international team from Japan and USA showed, for the first time, evidence of extraterrestrial origin ${ }^{13} \mathrm{C}$-enriched sugars from carbonaceous chondrites in two meteorites (NWA801 and Murchison) by means of gas chromatography-mass spectrometry (GC-MS) [134].

In December 2014, aiming to verify the panspermia hypothesis associated with the origin of life and molecular chirality on Earth, the Hayabusa-2 spacecraft (Japan Aerospace Exploration Agency: JAXA (Chofu, Tokyo, Japan)) launched to land on the surface of 'Ryugu', which is one of the carbon-rich asteroids. In December 2020, on the way to the next target (1998KY26, a rapidly spinning asteroid), the spacecraft delivering a small capsule, called Urashima's treasure chest, containing organic and gaseous ingredients for life, had just returned to Earth [135]. Importantly, the substances captured from the sub-surface of Ryugu did not contain any contaminations existing on Earth. Japanese researchers are analyzing all of the ingredients. The team anticipates evidence of extraterrestrial-origin molecular substances associated with preferential chirality if the substances are chiral.

\subsection{Terrestrial Origins of Life and Chirality}

In 1953 and 1959, Miller and Urey reported production of nearly forty amino acids including glycine, $\alpha$-alanine, $\beta$-alanine, aspartic acid, and so on, by applying electric discharge under reducing atmosphere containing $\mathrm{CH}_{4}, \mathrm{NH}_{3}, \mathrm{H}_{2} \mathrm{O}$, and $\mathrm{H}_{2}$ [136,137]. The system is regarded as an open-flow, recycling chemical reaction system that is experimentally testable at ordinary lab level to mimic primitive Earth. Although the product chirality was not characterized at those days, the electric discharge was thought be, at least for the author, an unpolarized high-energy electromagnetic force that produces racemic amino acids. In 2013, Bada reviewed his insights of prebiotic chemistry under the volcanic condition [138].

In 2017, a Japanese team reported an astonishing result that thundering is generating $\gamma$-ray, called atmospheric photonuclear reaction [139]. The thundering acts as a naturally occurring accelerator, which might ubiquitously generate in primordial Earth, and is regarded as a realistic electric discharge in the Miller-Urey experiments [136,137]. The naturally occurring $\gamma$-ray is responsible for several photonuclear reactions. When $\gamma$-ray collides to the atmospheric ${ }^{14} \mathrm{~N}$ molecule, radioactive ${ }^{13} \mathrm{~N}\left(t_{1 / 2} 598 \mathrm{~s}\right)$ and neutron $\left(t_{1 / 2} \sim 890 \mathrm{~s}\right)$ will be generated. The unstable ${ }^{13} \mathrm{~N}$ causing $\beta^{+}$-decay reaction produces stable ${ }^{13} \mathrm{C}$, positron, and neutrino. The neutron with ${ }^{14} \mathrm{~N}$ produces radioactive ${ }^{14} \mathrm{C}\left(t_{1 / 2} 5700 \pm 30 \mathrm{yr}\right)$. The unstable radioactive ${ }^{15} \mathrm{~N}\left(t_{1 / 2} 122 \mathrm{~s}\right)$ by capturing the neutron re-generates $\gamma$-rays. The Japanese team detected $\gamma$-rays at $0.511 \mathrm{MeV}$ regenerated by electron-positron annihilation. These scenarios infer that all electron, positron, neutron, and radioactive atoms may become parity-violating handed physical sources.

A non-naturally occurring radioisotope ${ }^{60} \mathrm{Co}$, which is synthesized from stable isotope ${ }^{59} \mathrm{Co}$ by capturing one neutron only in a nuclear reactor, cannot contribute to the left-right imbalance on primordial Earth. However, naturally occurring, long-lifetime radioisotopes ${ }^{235} \mathrm{U},{ }^{238} \mathrm{U},{ }^{232} \mathrm{Th},{ }^{87} \mathrm{Rb}$, and ${ }^{40} \mathrm{~K}$, embedded to continental crust, mantle, oceanic crust, and sediment of Earth contribute in principle to the left-right imbalance through the $\beta^{-}$-decay channels radiating handed elemental particles and radiating $\gamma$-rays [140-142]. 


\subsection{Extraterrestrial Origins of Circularly Polarized Radiation Sources}

On the other hand, scientists have long argued that several kinds of circularly polarized electromagnetic sources, including $\gamma$-ray, $\mathrm{X}$-ray, and vacuum-UV in the universe, are responsible for the biomolecular handedness [4-9,13-16]. Alternatively, a subtle imbalance of L- and D-amino acids as tiny chiral chemical seeds significantly catalyze asymmetric chemical reactions to yield carbohydrates with a high ee, leading to the handed biomolecular world [48]; a nearly racemic substance in $10^{-5} \%$ ee attained a nearly $~ 100 \%$ ee when the Soai reaction was regarded as a testable model of the chemical evolution of chirality in prebiotic eons [143,144].

When these substances are homogeneously dissolved in solutions, a probability of $\mathrm{CP}$ light (photon chirality) that reacts with racemic and prochiral biomolecular is, at most, one-chance with quantum efficiency of 1.0 by assuming the Beer-Lambert law. We assume that non-rigid, efficient, photonic resonators adaptable to photon chirality is possibly suited for recycling $\mathrm{CP}$ light-matter interactions, such as whispering gallery mode (WGM).

\subsection{Coacervate Hypothesis}

In the 1920s-1930s, Oparin [23] and Haldane [145] proposed the coacervate hypothesis, which is a prototype of living cells during the chemical evolution of life. The coacervate refers to sphere shape, cell-wall free colloidal droplets, from $1 \mu \mathrm{m}$ and $100 \mu \mathrm{m}$ in diameter, surrounded by fluidic water. The colloids comprising organic substances were postulated to spontaneously lead to a metabolism after a prolonged time. The hypothesis relies on a scenario of spontaneous self-organization arising from non-covalent interactions, such as hydrogen bonds, electrostatic, van der Waals, dipole-dipole, and so on. The hypothesis was mostly abandoned because the closed coacervate systems in an equilibrium state did not evolve living organisms.

In 2020, Lu and Spruijt demonstrated experimentally and theoretically how immiscible multicomponent droplets consisting of oppositely charged polyelectrolytes in the presence of several additives undergo a spontaneous formation of ca. 1-10 $\mu \mathrm{m}$ size multi-phase coacervates, with help from interfacial tensions and concentration of salts [146]. The multiphase coacervates dispersed in water containing salts spontaneously propagate to hierarchically self-organize cellular structures with time. The coacervates hypothesis is not an old-fashioned concept. A remodeled coacervates hypothesis sheds light on a new insight of how non-equilibrium colloidal particles in fluidic solutions propagate spatiotemporally [146]. In-situ observations of spatiotemporal propagation behaviors associated with a preferred handedness in chirogenesis of coacervates, using sophisticated circular dichroism (CD) and circularly polarized luminescence (CPL) microscopy, are challenging [147-152].

\subsection{Colloids}

Colloid science has a long history [153]. Colloids involve micelles, vesicles, shapecontrolled polymers, molecular and macromolecular aggregates, emulsion of polymers, core-shell nanoparticles, spherulite, microgel, and possibly, coacervate. In 1919, le Chatelier hypothesized that the size of colloids ranges from $1 \mathrm{~nm}$ to $1000 \mathrm{~nm}$ [154]. In the 1920s, makromolekül (macromolecule) was categorized to one of the colloids because the makromolekül hypothesis proposed by Staudinger was controversial [155]. Although the concept was established in the 1930s, charged colloids made of macromolecules and/or polymers did not appear to be well-recognized in those days. It is obscure whether Oparin and Haldane recognized in those days that the coacervate hypothesis was intimately connected to colloid science, macromolecular/polymer science, and supramolecular science. In 1952, Terayama proposed polyion complexes in water of oppositely charged water-soluble polyelectrolytes, nowadays, widely known as the colloid titration method [156]. Non-charged co-colloidal polymers, however, did not appear to be well-recognized until recently. It is still unclear how co-colloidal chiral polymers propagate with time. 


\subsection{Ostwald Ripening and Viedma Ripening}

In 1990, Kondepudi et al. found that, from saturated solution of $C_{3 \mathrm{v}}$-symmetric achiral sodium chlorate $\left(\mathrm{NaClO}_{3}\right)$ in water, $\mathrm{L}$ - or D-crystal in nearly $100 \%$ ee is dominantly generated in a beaker solely by mechanically stirring. Contrarily, no preference in the L-D crystal was obtained under stagnant condition [157]. The homochiral L- or D-world in the beaker is determined by the by-chance mechanism. Martin et al. indicated that damages in the first-generation crystals, led by the stirring, can produce numbers of the second-generation crystals in smaller size, which are responsible for the production of Lor D-crystal in a vessel [158]. The initial chirality in the first-generation crystals act as the seed of chirality in the second-generation crystals during mechanical stirring, leading to autocatalytic homochiral crystallization. It is noted that hydrodynamic convection flow as mimic model of Coriolis force was not responsible for the L-or-D crystallization [158].

These studies stimulated another autocatalytic homochiral crystallization, known as the Viedma ripening. In 2005, Viedma observed attrition-enhanced de-racemization phenomenon in a mixture of solid/liquid of enantiomorphous crystals using $\mathrm{NaClO}_{3}$ [159]. In 2008, Viedma and coworkers developed this approach to evolution of homochiral crystal D- or L-aspartic acid in the presence of salicylaldehyde as a catalyst at elevated temperatures $\left(90-160^{\circ} \mathrm{C}\right)$ [160]. Enantiomerically pure aspartic acid that is rapidly racemized upon heating is assumed to be a realistic amino acid existing on the primordial Earth.

Possible mechanisms underlining should involve four steps [161]: (i) Ostwald ripening with an initial chirality with help from the gliding to introduce crystal damage/defects and non-racemic additives; (ii) enantiospecific aggregation to a larger aggregation with the same chirality; (iii) a breakage of the aggregates; and (iv) racemization. Ostwald ripening with chirality is a dynamic process of chirality crystal growth and dissolution due to the crystal-size dependent solubility. A larger size chiral crystal has a lower solubility due to a smaller specific surface area, conversely, a smaller size chiral crystal has a higher solubility due to a large specific surface area.

In 2007, Viedma tested the parity-violation hypothesis of whether handed crystals ( $\mathrm{L}$ or D) using $\mathrm{NaClO}_{3}$ and $\mathrm{NaBrO}_{3}$ are generated [162]. Heavy $\mathrm{Br}$ atom was expected to boost the parity-violation effect obeying 5-6 power dependency of the weak neutral current based on a large spin-orbit coupling. Systematic experimental results of mirror symmetry breaking on a macroscopic level, however, did not support the parity-violation hypothesis. The results were ascribed to unidentified cryptochiral impurity existing in environmental conditions. The crystallization protocol is, possibly, very susceptible to external impurities as chiral seeds, rather than the weak neutral current effect.

These studies infer to us that, by controlling the dynamic process between chirality and non-chirality at the solid-liquid interface, an alternative heterogeneous chirogenic system comprising colloid and its dispersion solvent is possible, when proper colloidal polymers with chiral liquefied media are chosen.

\subsection{Optofluidics Connecting to Colloids and Circularly Polarized Light}

In 1986, Qian et al. reported the first laser emission from free-falling droplets doped with red-color dyes upon excitation of a $532 \mathrm{~nm}$ laser based on WGM mode, or morphological dependent resonance mode, whereas rhodamine 590 and rhodamine 640 were used as dyes [163]. In 2006 to 2010, the optofluidics, which is analogous to the corresponding solid-state optical devices, was coined for a conceptional fusion of integrated optics and microfluidics [164-167]. Microfluidics is an essential science of precisely controlling and manipulating fluidic medium in the range of ten-to-hundred $\mu \mathrm{m}$ in diameter. One can design unique $\mu \mathrm{m}$-scale liquid-based optical devices with a greater flexibility; for example, it is easy to: (i) tailor several optical properties of the fluidic medium, such as refractive index (RI), wettability, and viscosity; (ii) construct optically smooth colloid-liquid interfaces; and (iii) confine massless light into an optical resonator. Moreover, Whitesides, Psaltis, and coworkers demonstrated that ultralow threshold dual-color lasing from rhodamine 560 and rhodamine 640 in $20-40 \mu \mathrm{m}$ size droplets of benzyl alcohol $\left(n_{\mathrm{D}}=1.54\right)$ is possible 
when dispersing in $\mathrm{C}_{7} \mathrm{~F}_{15} \mathrm{OC}_{2} \mathrm{H}_{5}$, which is a low RI fluorinated solvent with $n_{\mathrm{D}}=1.29$ [168]. Note that $n_{\mathrm{D}}$ means an average RI value at 589.0 and $589.6 \mathrm{~nm}$ of doublet D-lines arising from vapor of atomic sodium. The lasing droplet device is realized based on the WGM scheme $[163,168,169]$. The idea of optofluidics was recently realized as Varioptic ${ }^{\circledR}$ from Corning ${ }^{\circledR}$ (Corning, NY, USA), which is a commercial product of variable focus liquid lens. The Fabry-Pérot resonator in a planar microfluidic geometry using Bragg-type grating reflectors shows a resonantly enhanced transmission with a sharp peak at a well-tuned RI in a fluidic medium [170].

In a light-harvesting plant system, stroma in chloroplasts is adaptable to any alterations, such as osmotic pressure, $\mathrm{Mg}^{2+} / \mathrm{K}^{+}$ions, sunlight intensity, temperature, and so on [171-174]. The stroma may work as naturally occurring, self-tuning optofluidic medium. The underlying mechanism teaches that, to maintain the non-CP and CP light-driven photophysical and biological properties, the chirally assorted macro-colloids surrounding optofluidic stroma appear key [171-174]. Likewise, the adaptability and flexibility to any alterations in chemical and physical biases are crucial in the chemical evolution and propagation of life.

The noticeable advantages facilitate anyone to fabricate the low-reflection-loss chromophoric and/or luminophoric colloidal molecules and polymers with high RIs by surrounding a fluidic medium with a lower RI to resonantly boost chiroptical spectral responses in the ground and the photoexcited states. Based on the optofluidics, the colloidal polymers in the ground and photoexcited states as a function of RI of the surrounding solvents are rarely studied. We showcase several optofluidic effects of colloidal polymers in the ground and photoexcited states by tuning the RI of the solvents in the following.

\subsection{Microdroplets and Aerosols - Prebiotic Chemical Reactors}

Atmospheric $\mu \mathrm{m}$-sized aerosols and $\mu \mathrm{m}$-sized droplets called microdroplets in water offer unique chemical and photochemical reactors by concentrating molecules, which serve several key precursors, such as oligopeptides and ribonucleosides in the prebiotic Earth [175-177]. The $\mu \mathrm{m}$-sized aerosols and $\mu \mathrm{m}$-sized microdroplets are regarded as realistic models testable of the coacervate hypothesis on a laboratory scale.

Recently, researchers became aware of the fact that various chemical reactions and processes in the microdroplets are significantly accelerated by several orders of the magnitudes, compared with the corresponding reactions and processes in the bulk phase [178]. These reactions and processes confined in the microdroplets differ from the conventional chemistry in homogeneous solutions. The reactions involve protein unfolding [178], helix formation [179], phosphorylation in ribonucleosides [176,177], production of nanostructures [180], autoreduction [181], and so on. Although the oil/water, air/water, and silica/water interfaces are used as the platforms, the cause of the acceleration mechanisms in these microdroplets is as a matter of debate.

To address the issue, in 2018, Zare et al. designed-to experimentally and computationally study the distribution and photoluminescence (PL) polarization anisotropy of rhodamine $6 \mathrm{G}$ as a PL probe doped into microdroplet oils $\left(3 \mathrm{M}^{\mathrm{TM}}\right.$ Fluorinate ${ }^{\circledR}$ FC-40-a mixture of two perfluoroalkyl amines, $\left.n_{\mathrm{d}}=1.29\right)$ dispersed in water $\left(n_{\mathrm{d}}=1.33\right)$ [182]. They found that, when a radius of the microdroplet increases and a concentration of rhodamine $6 \mathrm{G}$ decreases, the density of rhodamine 6G is significantly higher on the surface than in the center of the microdroplets and that the ratio of the surface density to that of the center grows. Moreover, PL polarization anisotropy on the surface of the microdroplets is significantly large, indicating that rhodamine $6 \mathrm{G}$ is well-aligned on the surface. The reduced entropy affects the significant change in the free-energy for the reactions [182].

The concept of optofluidics is applicable to photoexcited microdroplets and aerosols that are regarded as open-flow, non-equilibrium, volatile photochemical resonators. Relative RIs of organic microdroplets in water and aerosols in air are $n_{\mathrm{d}}$ (droplet) $/ n_{\mathrm{d}}($ water) $=1.4-1.6 / 1.33$ and $n_{\mathrm{d}}$ (water solution) $/ n_{\mathrm{d}}$ (air) $\geq 1.33 / 1.00$, respectively. When prochiral substances in the photoexcited microdroplets and aerosols are concentrated on their sur- 
faces, one can anticipate WGM-driven accelerated photochemical reactions in absolute asymmetric synthesis (AAS) or absolute photochirogenesis (APC) with a CP light source in the absence of an external magnetic field and unpolarized light in the presence of an external magnetic field.

\subsection{Our Hypothesis for Chirogenesis and Photochirogenesis}

As mentioned above, the multiple concepts of panspermia and coacervate hypotheses, $g^{*}$ value, bi-furcation model, circularly polarized (CP)-light driven photochirogenesis, optofluidics, and WGM stimulated us to investigate open-flow, non-equilibrium colloidal systems composing of artificial chain-like polymeric chromophores and luminophores dispersed in optofluidic biomolecular liquefied chiral terpenes, as chiral foods and beverages. Moreover, we explored a possibility of AAS or APC of the artificial coacervate as a suspension state in achiral organic solvents upon excitation of a wavelength-dependent $\mathrm{CP}$-light source as a model of cosmos-origin chiral electromagnetic forces. These external chirality-driven, open-flow systems allowed to mimic the tempo-spatial transcription of molecular chirality and light chirality in the cell-wall free colloids on Earth at ambient temperatures in the absence and presence of $\mathrm{CP}$ light sources.

The open-flow, dissipative structures connected to molecular motions is a curious subject in the present special issue [183]. This is beneficial because chirogenesis and photochirogenesis from optically inactive colloids of $\pi$-/ $\sigma$-conjugated polymers in the ground state and in the photoexcited state are easy to quantitatively characterize by means of CD, optical rotation dispersion (ORD), CPL, and CPL excitation (CPLE) spectroscopy. The $\pi$-/ $\sigma$-conjugated polymers are excellent chromophores and luminophores as probes in place of amino acids and sugars.

Our knowledge and understandings of the $\pi$ - $\sigma$-conjugated polymers at ordinary laboratories on the northern hemisphere of the spinning Earth could shed light on rethinking about possible scenarios of the first life associated with L-amino acids and D-sugars, regardless of terrestrial and extraterrestrial origins.

\subsection{Resonance Effects from Colloidal Systems in Optofluidic Media in the Ground and Photoexcited States}

In the following sections, we highlight the importance of optofluidics for achieving an efficient chirogenesis and an efficient photochirogenesis using several $\pi$-/ $\sigma$-conjugated polymers in UV-visible-near IR (NIR) regions. Particularly, a fine tuning of the RI value of the surrounding liquids allows us to resonantly boost chiroptical signals and photochemical reactions within a $\mu \mathrm{m}$-size colloidal polymers. To our knowledge, such comprehensive, systematic studies, showcasing marked resonance effects of chirogenesis and photochirogenesis in the realms of chiroptical spectroscopy, asymmetric chemistry, synthetic chemistry, polymer chemistry, colloid chemistry, photochemistry, photophysics, and materials chemistry, have not been reported yet.

All of the spectral analyses of colloidal substances in the ground and photoexcited states were conducted by practical chiroptical approaches using JASCO J-725/J-820/CPL200 spectrometers equipped with commercial and custom-made accessories. First, a classical ORD spectroscopy shows us, exactly, a left-right imbalance of chiral chromophores in the ground state as differences in relative RIs and/or light speeds between left- and rightcircular polarized light as a function of wavelength in a vacuum. Next, CD spectroscopy allows detecting a left-right imbalance of chiral chromophores in the ground state as a difference in absorption (attenuation) between left- and right-circular polarized light as a function of wavelength in a vacuum. Moreover, CPL spectroscopy can detect a left-right imbalance of chiral luminophores in the photoexcited state as a difference in radiation probability between the left- and right-CP light emission processes when unpolarized light is excited at a specific wavelength of the chromophore as a function of wavelength in a vacuum [184]. In an analogy to achiral photoluminescence excitation (PLE) spectroscopy, CPLE spectroscopy provides differences in absorption band profiles in the ground state responsible for the specific CPL signals in the photoexcited state solely by monitoring, at a 
specific CPL band, as a function of wavelength in a vacuum. Although CPLE spectroscopy is rare in the realm of chiroptical spectroscopy, we often apply this technique to clarify which unpolarized absorption and CD bands are responsible for CPL/PL bands, particularly, dual/multiple CPL bands with opposite chiroptical signs [185]. Importantly, CPL, CPLE, PL, and PLE spectroscopy cannot apply to non-luminescent and ultraweak luminescent chromophores. More importantly, achiral and 50:50 racemic mixture of chromophores in the ground state and luminophores in the photoexcited state reveal zero-signals because of any differences between left- and right-ORD, between left- and right-CD, between leftand right-CPL, and between left- and right-CPLE signals. Notably, we should pay attention to artifact signals when anisotropic specimens, oriented films, solid substrate with periodicity acting as additional linear polarizers are employed [186]. It is beneficial that, because $\mu \mathrm{m}$-size colloidal particles are randomly dispersed in fluidic medium that is similar to optically anisotropic molecules in homogeneous solutions, the chiroptical artifact is not a serious issue, even if an individual colloid is optically anisotropic.

\section{Colloid-Induced Chiroptical Enhancement and Aggregation-Induced Emission}

Firstly, we briefly touch upon two histories in the communities of artificial chiral chemistry, solution chemistry, and colloid/aggregation chemistry, including colloid-induced chiroptical enhancement (CIE) and aggregation-induced emission (AIE).

A photophysical transition from nearly non-emissive molecules dissolved in homogeneous solutions to highly photoluminescent colloidal particles dispersed in heterogeneous solutions was established as a well-known phenomenon in the last two decades [187,188]. In 2001, Tang et al. found an anomaly in the enhancement of quantum yield from aggregates made of 1-methyl-1,2,3,4,5-pentaphenylsilole [189]. The silole derivative revealed an abrupt enhancement in the quantum yield $(\phi)$ of PL by solely adding water from a weak $\phi \approx 0.06 \%$ in ethanol to high $\phi \approx 90 \%$ in water-ethanol cosolvents. The aggregation process is responsible for the enhanced quantum yield. The phenomenon was coined aggregation-induced emission (AIE). Soon, the origin of the AIE phenomenon was explained by restricted intramolecular rotations of multiple $\mathrm{C}-\mathrm{C}$ bonds of phenyl groups at peripheral position of the silole ring [190]. Either increasing solvent viscosity or decreasing solution temperature efficiently enables suppressing the rotational freedom at a molecular level in the aggregates. This idea was viable for several aggregates made of polyacetylenes substituted with floppy 1,1,2,3,4,5-hexaphenylsilole (HPS) in acetone-water cosolvents. In 2012, the aggregate of silole derivative carrying two long D-sugar-based tails in $n$-hexane-dichloromethane cosolvents showed the first AIE-circular dichroism (AIE-CD) in the ground state and AIE-circularly polarized luminescence (AIE-CPL) in the photoexcited state $[188,191]$. The Kuhn's dissymmetry ratio in the CPL spectrum $\left(g_{\mathrm{CPL}}\right)$ value [188] reached $\approx-0.32$ at $500 \mathrm{~nm}$, in which the degree of circular polarization was -0.16 . Similarly, the aggregate of tetraphenylethylene (TPE) substituted with two L-valines in a mixture of dichloroethane and methanol revealed AIE-CPL of $g_{\mathrm{CPL}} \approx-5 \times 10^{-3}$. Highresolution images of scanning electron microscope and transmission electron microscope (TEM) indicated that one-dimensional helical fibers of the TPE aggregate are responsible for the AIE-CPL and AIE-CD $[188,192]$.

We should touch on major differences between AIE-CD/AIE-CPL and CIE-ORD/CIECD/CIE-CPL/CIE-CPLE because both phenomena are related to significant amplification of chiroptical signals in the ground and photoexcited states.

First, AIE-chiroptical systems rely on significant suppressions of rapid intramolecular rotations among single bonds based on very low rotational/twisting barrier heights, which are responsible for thermally excited, non-radiation processes in the photoexcited and ground states. To achieve the suppressions at a molecular level, adding poor- or nonsolvent-like water to homogeneous solutions, e.g., tetrahydrofuran (THF) and acetone as good solvents, of the weakly emissive substances is the key. The poor-/non-solvent and good solvents are completely miscible in any volume fractions. Actually, the building blocks of AIE, such as TPE and HPS, are highly emissive in the solid states, owing to 
great suppression of molecular motions and vibrations, where they dissolved in good solvents, are weakly emissive due to rapid rotational/twisting mobilities. In my view, controlling RI values of a mixed co-solvents of poor-/non-solvent and good solvent does not appear to be a major factor. However, the $n_{\mathrm{D}}$ value might alter in response to volume fractions of THF-water cosolvents because a lower RI water $\left(n_{\mathrm{D}}=1.333\right)$ and a higher RI THF $\left(n_{\mathrm{D}}=1.407\right)$ are mixed together. Thus, we assume that AIE-chiroptical signals of the aggregates are possible, to maximize at specific volume fractions, namely, specific $n_{\mathrm{D}}$, of good-poor co-solvents, to satisfy a resonance condition.

Contrarily, CIE chiroptical systems are largely affected by relative RI values between colloidal polymers and the surrounding fluidic media. To rationally realize the resonance condition in chiroptical signals, we often use semi-flexible and rod-like chain-like chromophoric polymers with a narrower molecular weight distribution. These polymers are inherently highly emissive, even in dilute homogeneous solutions, and in solid films. The reason for the semi-flexible and rod-like polymers is to restrict rotational freedoms along huge numbers of single bond sets. These polymers ensure spontaneous association of well-ordered packing structure during a controlled slow addition of a poorer solvent to the polymer solution, monitored by the naked eye. This approach belongs to a fractionation technique, to isolate the desired molecular weight of polymer from a broad molecular weight distribution of synthetic polymers, during a two-phase separation phenomenon. In our case, fine tuning the RI value of poor and good cosolvents, however, is the key, because polymers in the colloids often involve the surrounding co-solvents and usually exist as a swollen state. Thus, we have to experimentally determine the best volume fraction, namely, RI value, of poor and good cosolvents by measuring CD spectral shape with CD signal magnitudes of the colloidal systems. However, more importantly, when a specific resonance condition in the colloidal system is established with help from the RI-tuned cosolvents, the tiny chiroptical signals are enhanceable by huge times of reflection at the colloid-liquid interfaces due to confinement of traveling light in line with WGM, as mentioned in the following section.

\section{Open-Flow, Non-Equilibrium Coacervate Hypothesis Meets Optofluidics}

Our early assumption had to be remodeled when we came across optofluidics-that is an influential combined concept, at least for us, between liquid-based microoptics and soft-matter based optical devices. A traveling light, massless photon energy source, enables to efficiently confine polarized light in the interior of a droplet in a fluidic medium. The confinement causes a recurring polarized light-matter interaction within the droplet and /or at the droplet-liquid interfaces. An extension from optofluidics to chiral optofluidics might be applicable to chirogenesis and photochirogenesis from (at least) 1 to 100 $\mu \mathrm{m}$-sized colloidal particles, with a higher RI, by dispersing in a fluidic medium with a lower RI, although their RIs have to be tuned precisely. The medium is not restricted to fluidic media. Likewise, solidified media, such as glassy polymers and inorganic solids, are possible to use.

Linearly polarized light is a superposition of left $(l-)$ and right $(r-)$ circularly polarized (CP) light. The $l$ - and $r$-CP-light carry angular momenta of integer spins $( \pm \hbar)$, respectively [193-196]. Previously, Ghosh and Fischer [193] and Silverman et al. [194] simulated that the optical rotation from chiral liquids is boosted by several orders of magnitude when a coupled geometry of multiple prisms is filled with optically active biomolecular liquids, such as limonene, carvone, and a mixture of camphorquinone and methanol. The concept of AAS with light, one of the curious topics in the present special issue of Symmetry [197], is classified to: (i) absolute asymmetric photosynthesis; (ii) photodestruction; and (iii) photoresolution [47-49].

According to a theory led by Mortensen et al., the slow light of colloidal particles, by filling with a liquid medium, could enhance the CD signal at the edges of the optical band gap $[195,196]$. This theory is verified by enhanced ORD signals in a fluidic medium with a tuned RI. ORD spectroscopy allows us to detect differences in light speeds between $l$-and 
$r$-CP light as a function of incident light. In an analogy of the ORD signal enhancement, one can assume that $\mathrm{CD}$ spectrum as a function of wavelength of incident $l$ - and $r$-CP light is possible to detect enhanced differences in light speeds between $l$ - and $r$-CP light when optically active colloids are dispersed into a proper liquid medium by solely tuning RI.

These seminal theories of optofluidics and our CIE chiroptical phenomena prompted us to focus on the chiral optofluidic medium suited to $\mu \mathrm{m}$-sized colloids, consisting of luminous $\sigma-/ \pi$-conjugated polymers. With this mind, we designed several optically active fluidic media containing limonene, $\alpha$-pinene, and other biomolecular solvents [54-57,198-212]. Moreover, we expected an efficient APC when the CP-light source, with sense, was employed as a function of excitation wavelength [54-56].

Herein, we highlight a series of CIE-CD, CIE-ORD, CIE-CPL, and CIE-CPLE experiments from the colloids of $\pi$ - $/ \sigma$-conjugated polymers and co-colloids consisting of both $\pi$ and $\sigma$-conjugated polymers, in terms of artificial models of open-flow, non-equilibrium coacervates in the ground state and the photoexcited state. These colloidal polymers are readily dispersed in a tuned optofluidic medium by inspiring the possible scenarios of the homochirality questions on Earth. By controlling RI of the surrounding medium, the $\mu \mathrm{m}$-size colloidal $\pi$-/ $\sigma$-conjugated polymers in the optofluidic medium resonantly reveal enhanced characteristics of chirogenesis and photochirogenesis [198-212]. The chirogenesis is susceptible to chiral optofluidic medium. An ability of APC from achiral colloidal polymers in achiral optofluidic medium is possible by choosing tandem factors of wavelengths and a sense of $\mathrm{CP}$ light sources [54-56], which are purely massless chiral photons carrying integer spins of $\pm \hbar[54-56,213-215]$.

\section{CIE-CD in the Ground State and CIE-CPL in the Photoexcited States}

\subsection{Steady-State CD and CPL Spectroscopies}

Steady-state CPL spectral datasets tell us information of the short-lived chiral species in the photoexcited state, while steady-state CD and ORD spectral datasets indicate long-lived chiral species in the ground state. Here we applied Kasha's rule and Jablonski diagram $[216,217]$ to the nature of optically active luminophores and chromophores (Figure 1). The short-lived species $\left(S_{1}, S_{2} \ldots\right)$ upon excitation of unpolarized light are generated on the timescale of $\sim 10^{-15} \mathrm{~s}$ based on the Franck-Condon principle [218]. The shortlived species, followed by non-radiative relaxation processes with vibrational modes, relax spontaneously to the lowest vibronic state $\left(S_{1}\right.$ with $\left.v^{\prime}=0\right)$, such as the metastable photoexcited state at the time scales of $10^{-11}-10^{-12} \mathrm{~s}$. Finally, a spontaneous relaxation occurs from the low-entropy handed $S_{1}$-state $\left(v^{\prime}=0\right)$ to the high-entropy, non-handed $S_{0}$-state $(v=0,1$, $2 \ldots$ ), proven by CPL signals with a lifetime of $10^{-9}-10^{-6} \mathrm{~s}$.

Absolute temperature of $300 \mathrm{~K}$ equals to $0.60 \mathrm{kcal} \mathrm{mol}^{-1}\left(0.0259 \mathrm{eV}\right.$ and $\left.208 \mathrm{~cm}^{-1}\right)$. When luminescent molecules and polymers are photoexcited at $400 \mathrm{~nm}$ in a vacuum, the $400 \mathrm{~nm}$ light energy corresponds to $35,970 \mathrm{~K}\left(3.10 \mathrm{eV}, 71.5 \mathrm{kcal} \mathrm{mol}^{-1}, 25,000 \mathrm{~cm}^{-1}\right)$. CPL/CPLE and CD/ORD spectroscopy, thus, allow to dictate different chiral information in the photoexcited and ground states, respectively. Although CPL signals dictate a temporal short-lived chiral species at 35,970 K, CD and ORD spectral data provide information of long-lived chiral species at $300 \mathrm{~K}$. When one can ensure the identity chiral species in the ground and photoexcited states, enantiomeric pairs of rigid luminophores and rigid chromophores are needed to restrict by intra-/intermolecular rotations at $35,970 \mathrm{~K}$. Optically active luminescent colloids are candidates to design WGM-based chiroptical resonators between a pair of (+)- and (-)-CIE-CPL signals. 


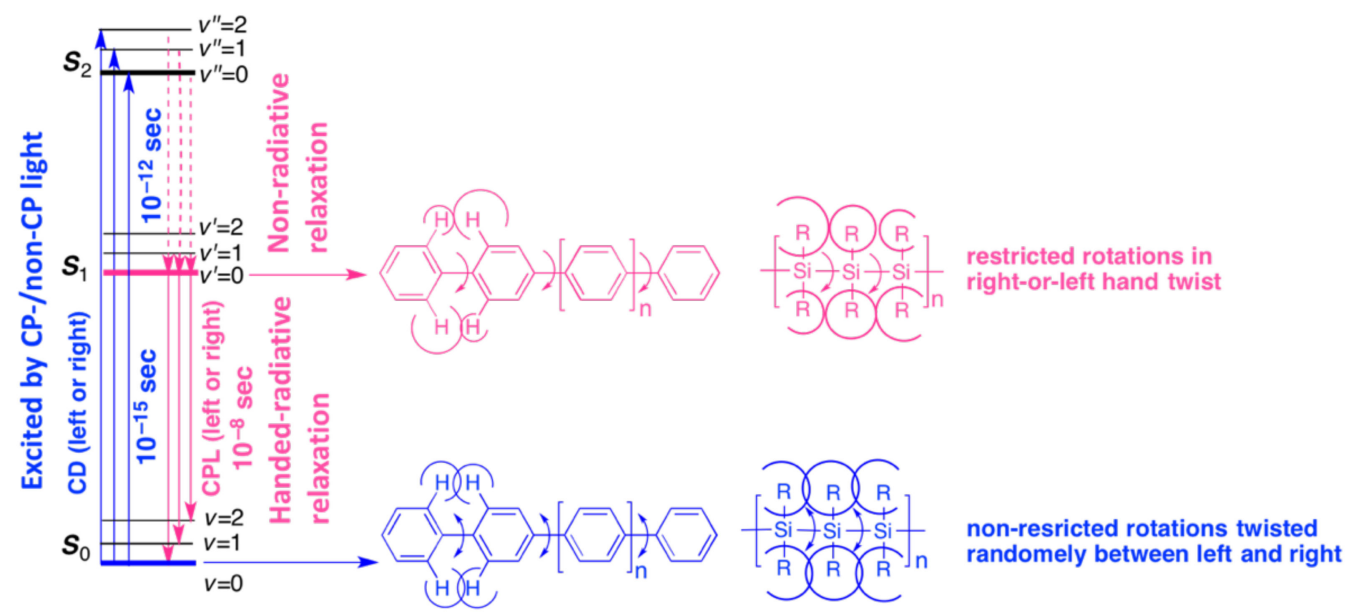

Figure 1. A brief concept of the modified Jablonski diagram, Kasha's rule, and Franck-Condon principle $[212,216-218]$ of chiral $\sigma-/ \pi$-conjugated chromophores with/without rotational freedom in the absence of optical resonator, whispering gallery mode (WGM), and optofluidic effects. Singleheaded arrow stands for restricted rotation clockwise (CW) or counter-clockwise (CCW); doubleheaded arrow indicates a free rotation CW and CCW. Modified from an original article [199] and a book chapter [212].

This idea predicts that, although a non-rigid chiral luminophore arising from substantial rotational freedom in the ground state does not reveal detectable CD and weak CD signals known as cryptochirality, the luminophores does not emit clearly CPL signal, and emits, apparently, no detectable CPL with the corresponding unpolarized PL due to an equal probability of left- and right-hand chiral structures in the photoexcited state. However, if a non-rigid chiral luminophore produces certain chiral colloids induced by the restricted rotational freedom, one can detect the spatiotemporal chiral colloid as CIE-CPL signal as well as CIE-CD and CIE-ORD signals. One can detect steady-state CIE-CPL signals because the lifetime of the spatiotemporal species is on the order of $\approx 1-10 \mathrm{~ns}$.

One may question whether the chiral optofluidics is valid for CIE-CD and CIE-CPL beyond an extension of AIE-CPL and AIE-CD. To prove the idea that the concept is generally applicable to a wide range of colloidal system suspensions in the fluidic media, we had to elucidate whether the chiroptical amplitudes in the resulting CD and CPL signals from several colloidal polymers were resonantly enhanced at specific RI values of the surrounded media. However, we had to consider serious concerns of the suspension heterogeneous systems. This is because anisotropic films and the vortex flowing from the chromophores and luminophores often cause unfavorable chiroptical artifacts and apparent sign inversion, as well as alteration in the absolute magnitudes in the CD and CPL signals [186]. To practically solve these artifacts, the use of optically anisotropic colloids dispersed in isotropic fluidic medium is one candidate. This approach is routinely applied to artifact-free CD and CPL spectral measurements by dissolving optically anisotropic molecules by dissolving in isotropic solution, although inherent anisotropic molecular information is lost. This is similar to routinely obtaining a solution of ${ }^{1} \mathrm{H}$ - and ${ }^{13} \mathrm{C}$ NMR spectra to measure highly anisotropic molecules in homogeneous solutions by rotating sample tubes and solid-state ${ }^{13} \mathrm{C}$ NMR spectrum of an anisotropic solid sample, by rapidly rotating sample tubes with a magic angle $\left(54.7^{\circ}\right)$ to cancel anisotropic terms.

Therefore, extremum wavelengths, chiroptical amplitudes, and signs in CPL/CPLE/CD/ORD signals, as well as those in PL excitation (PLE) and PL signals are not significantly affected by Rayleigh-Mie scatterings in proportion to $\lambda^{-4}$ ( $\lambda$ : wavelength of light in a vacuum). The scatterings often cause an unfavorable increment and apparent spectral shift embedded into the background UV-visible spectrum. Instrumental knowledge is crucial to characterize inherent chiroptical signals from optically active colloids in the ground and photoexcited states. It is noted that the RI value alters as a function of wavelength. ORD spectroscopy can measure the difference in RI between left (l)- 
and right $(r)$-CP light. Unpolarized light is a superposition of $r$-CP and $l$-CP light. Fine tuning RIs of colloidal particles and the surrounding medium is key when the idea of the chiral optofluidics is applied to CIE-driven chiroptical enhanced signals. We showcase several examples in the following sections.

\subsection{Gigantic Enhanced CD, ORD, and CPL from Colloidal Optically Active Helical Poly-silanes-Importance of Controlled RI in Optofluidic Medium}

Herein, we showcase, for the first time, a clear example that a precisely controlled RI of the surrounding medium, which is the essential concept of optofluidic, is critical to boost CIE-CD, CIE-ORD, and CIE-CPL signals for colloidal particles of several helical polysilanes. We tested poly\{p-(S)-2-methylbutoxypheneyl-n-propylsilane $\}$ (1S) [219], poly(n-decyl-(S)-3-methylpentylsilane) (2S) [220] poly(n-decyl-(S)-2-methylbutylsilane) (3S) [221], poly(n-dodecyl-(S)-2-methylbutylsilane) (4S) [21,199,221], and poly(n-dodecyl(R)-2-methylbutylsilane) (4R) [21,199] (Figure 2). Poly(n-hexyl-(S)-2-methylbutylsilane) (5S) [208-210,222,223] and poly(n-hexyl-(R)-2-methylbutylsilane) (5R) [208-210,222,223] were used as helical scaffoldings of producing chiral co-colloidal particles with several optically inactive $\pi$-conjugated polymers.
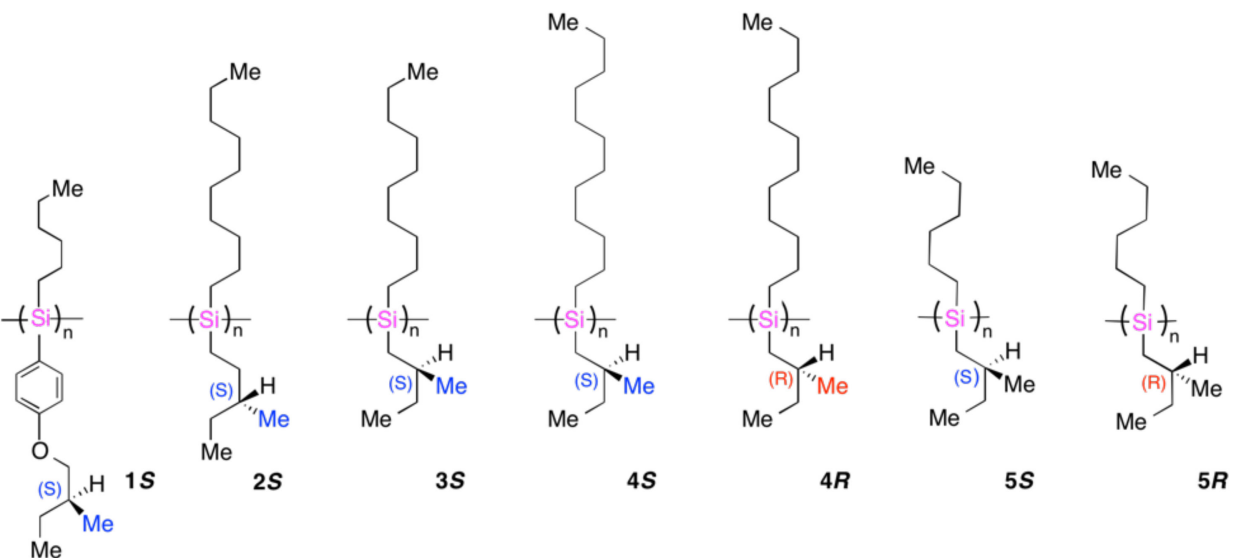

Figure 2. Chemical structures of semi-flexible helical alkylarylpolysilane (1S) and rod-like helical dialkyl polysilanes (2-5) carrying enantiomerically pure chiral substituents.

First, we highlighted the colloidal $3 S$ as a function of RI value of the surrounding cosolvents, by assuming that RI value of the colloidal $3 S$ is $n_{\mathrm{D}} \approx 1.7$ [199]. The CIE-CD, CIE-ORD, and CIE-CPL spectra of the $3 S$ are shown in Figure 3a-c. From Figure 3a, the $3 S$ clearly shows negative exciton couplet CD bands at the lowest Si $\sigma-S i \sigma^{*}$ band at $323 \mathrm{~nm}$. The Kuhn's dissymmetry ratio in the CD spectrum $\left(g_{\mathrm{CD}}\right)$ attain -0.31 at 325 $\mathrm{nm}$ and +0.33 at $313 \mathrm{~nm}$, respectively. These $g_{\mathrm{CD}}$ values are comparable to $\approx 16 \%$ left $(l)$-circular polarization and $\approx 17 \%$ right $(r)$-circular polarization, respectively, while pure $l$ and $r$-circular polarizations are $g_{\mathrm{CD}}= \pm 2.0$, respectively [224]. 

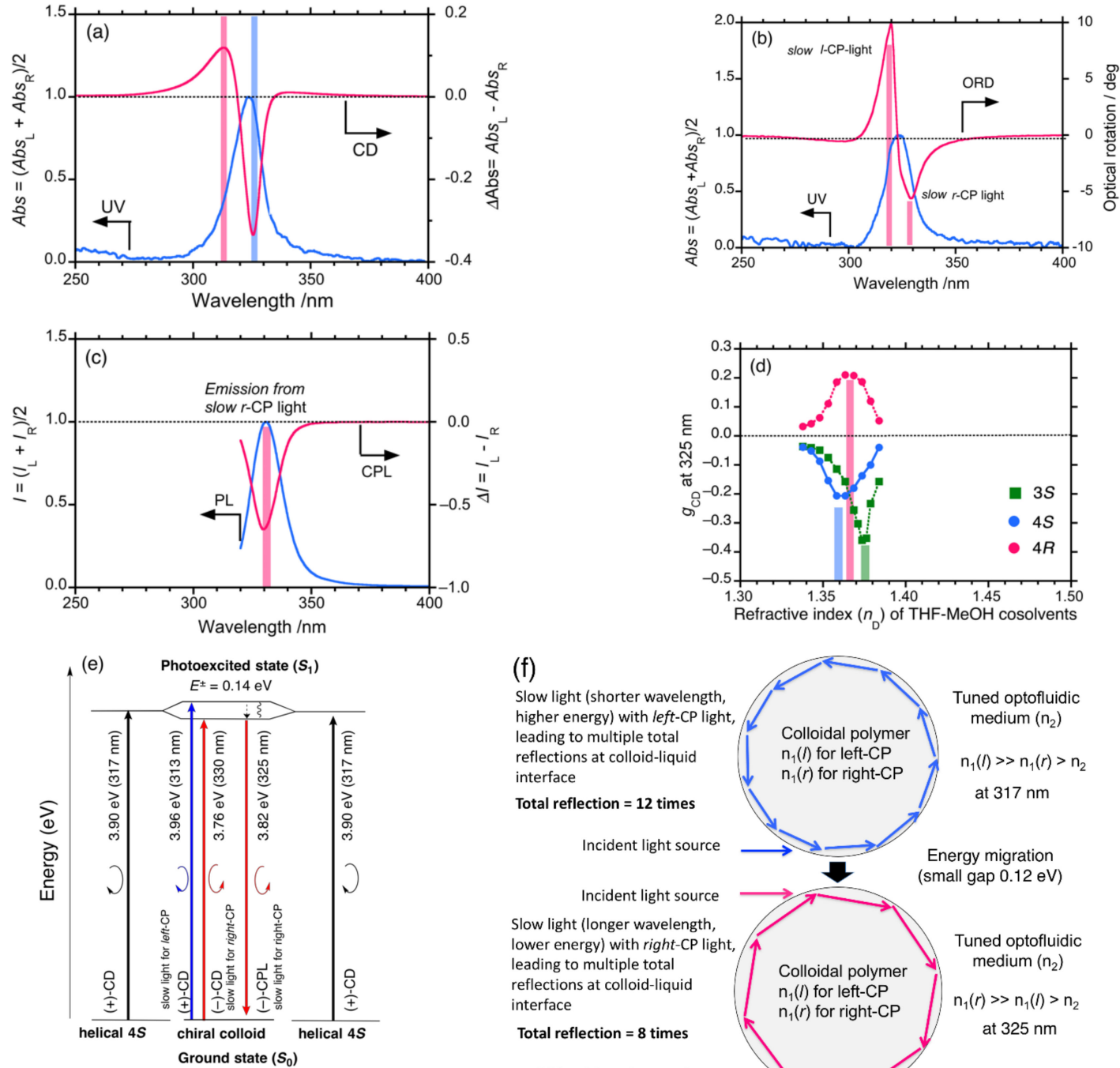

(f)
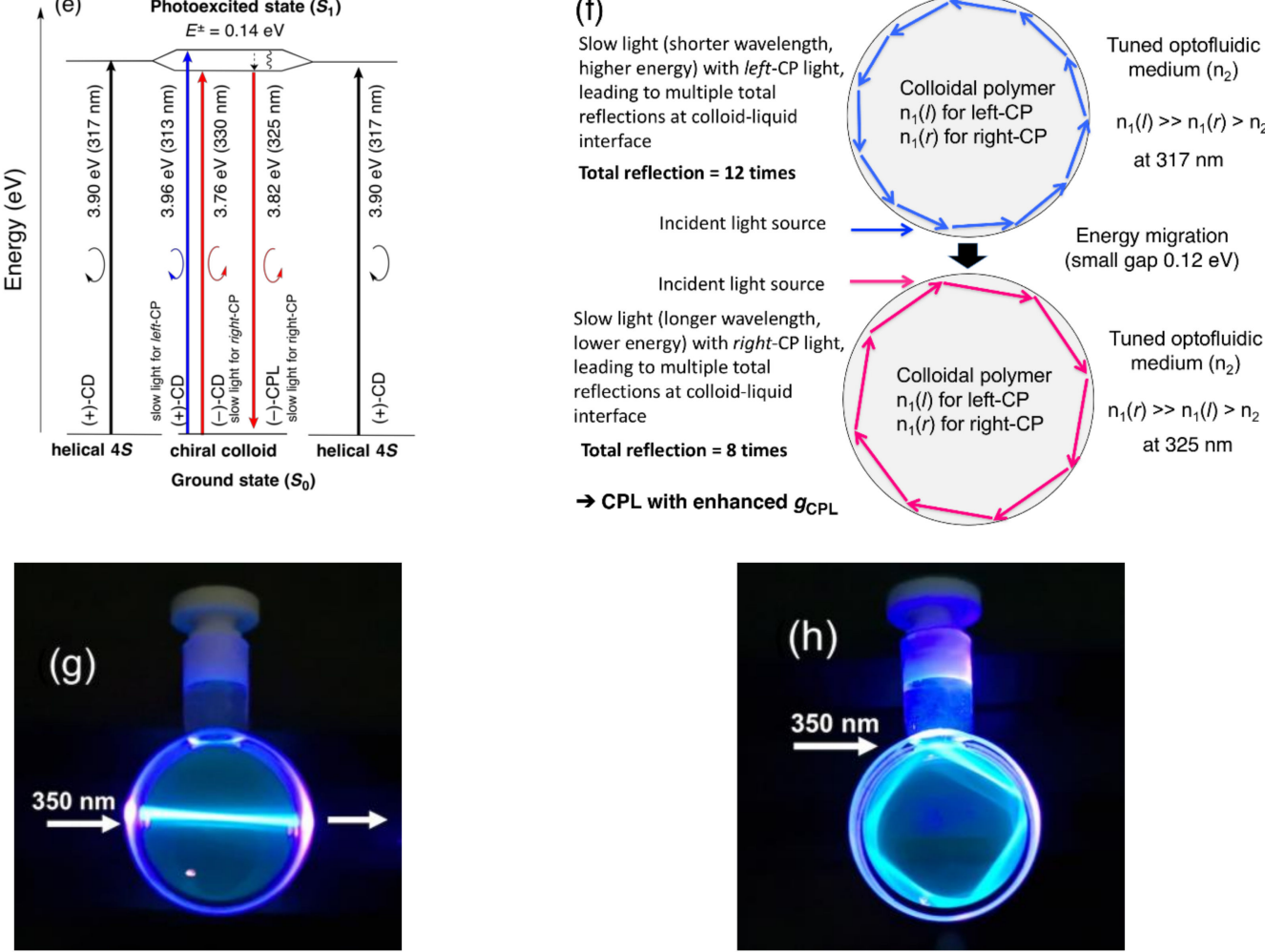

Figure 3. (a) Colloid-induced enhancement circular dichroism (CIE-CD) and UV spectra; (b) CIE optical rotation dispersion (CIE-ORD) and UV spectra; and (c) CIE circularly polarized luminescence (CIE-CPL) and PL spectra of the $3 S$ colloids; (d) The Kuhn's dissymmetry ratio in the CD spectrum $\left(g_{\mathrm{CD}}\right)$ of the colloidal $3 S, 4 S$, and $4 R$ as a function of refractive index (RI) in methanol-tetrahydrofuran (THF) cosolvents. The CIE-CD and CIE-CPL spectra are normalized by Kuhn's dissymmetry ratio in the ground and the photoexcited states [212,224,225]; (e) Schematic Jablonski diagram of the 3S; (f) a possible explanation for the CIE-CPL by confining left $(l)$ - and right $(r)$-circularly polarized (CP) light in the $3 S$ in optofluidic medium with a tuned RI [199]. Modified from an original article [199] and a book chapter [212]; (g) incident UV light at $\sim 0^{\circ}$ of the cuvette surface travels a very dilute $\mathrm{CHCl}_{3}$ solution of stilbene 420 upon excitation of unpolarized light at $350 \mathrm{~nm}$ of cylindrical quartz cuvette (22 mm in diameter, $1 \mathrm{~mm}$ in thickness of cell wall, $10 \mathrm{~mm}$ in cuvette optical path); (h) incident light at $\sim 50^{\circ}$ of the cuvette surface infinitely travels the stilbene solution, undergoing to WGM by four-time reflections per one-cycle at the $\mathrm{CHCl}_{3}$-quartz interfaces. 
As shown in Figure 3b, the colloidal $3 S$ clearly shows negative bisignate ORD spectra

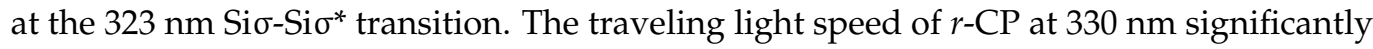
slows down compared to the traveling speed of $l-C P$ light at $320 \mathrm{~nm}$. Likewise, a traveling speed of $l-C P$ light at $320 \mathrm{~nm}$ slows down relative to the traveling speed of $r$-CP light at $320 \mathrm{~nm}$. Therefore, the relative RI value between $r$ - and $l$-CP light largely relies on wavelengths of incident light. A higher RI value results in slower CP-light along with shortening of wavelength because the wavenumber is unchanged, regardless of RI, by the conservation law of light energy.

The $3 S$ showed clearly single (-)-CPL signal at the $330 \mathrm{~nm}$ PL band associated with a small Stokes' shift of $466 \mathrm{~cm}^{-1}$, as depicted in Figure 3c. This small Stokes' shift indicates a very minimal structural reorganization owing to a very restricted rotational motion in the colloidal $3 S$ in the photoexcited state. The magnitude of $g_{\mathrm{CPL}}$ attained -0.65 at $330 \mathrm{~nm}$, corresponding to $\approx 33 \% r$-circular polarization, whereas the ideal $l$ - and $r$-circular polarization in the photoexcited state occurs at $g_{\mathrm{CPL}}= \pm 2.0$, respectively [225].

Both CIE-CD and CIE-CPL characteristics of the $3 S$ are nearly similar to those of the $4 S$ and $4 R$. Notably, the CIE-CD and CIE-CPL characteristics of the $3 S, 4 S$, and $4 R$ boost at the very specific $n_{\mathrm{D}}$ of mixed solvents of methanol and tetrahydrofuran (THF) (Figure $3 \mathrm{~d}$ ). The $g_{\mathrm{CD}}$ value of the $3 S$ is resonantly boosted at $n_{\mathrm{D}}=1.374$, which is a specific volume fraction of the cosolvent. Likewise, the $g_{\mathrm{CD}}$ values of the $4 S$ and $4 R$ are resonantly boosted at $n_{\mathrm{D}}=1.359$ and $n_{\mathrm{D}}=1.365$, respectively. The $n_{\mathrm{D}}$ dependent chiroptical resonance effects are an evidence of the chiral optofluidics, where noticeable differences in a traveling light speed between $l$ - and $r$-CP in the $\mu \mathrm{m}$-scale colloids in the ground state is evinced by tuning $n_{\mathrm{D}}$ of a liquid medium. The resonance effects are responsible for CIE-CD signals, followed by CIE-CPL signals in the photoexcited state.

From Figure $3 a-c$, a modified Jablonski diagram with chirality of the 35 associated with the Kasha's rule and exciton coupling theory is schematically illustrated in Figure 3e [199]. With the aid of the chiroptical resonator for selective $l$ - or $r$-CP light, a large difference in the RI between $l$ - or $r$-CP light is crucial.

In Figure 3f, we provide a plausible explanation for the CIE-CPL occurring in an optical resonator as a recurring WGM mode endowed with an efficiently confining $l$ and $r$-CPL in the $3 S$ with a high RI surrounded by optofluidic medium with a lower RI [199]. The optically active spherical colloids should work as chiroptical resonator for the respective $l$ - and $r$-CP light. As a result, efficient separation between $l$ - and $r$-CP light as radiation from the colloids is realized. Internal total reflection modes (Brewster angle and recurring numbers) at the colloid-liquid interface should be kept in mind.

Firstly, unpolarized light at $317 \mathrm{~nm}(3.90 \mathrm{eV})$, which is an equal mixture of $l$ - and $r$-CP light, excites simultaneously the colloidal $3 S$ with a high $n_{2}$ surrounded by the liquid with a lower RI. The RI value of $l$-CP light, $n_{1}(l)$, at $317 \mathrm{~nm}$ is higher than that of $r$-CP light, $n_{1}(r)$, at $317 \mathrm{~nm}$. The respective $n_{1}(l), n_{1}(r)$, and $n_{2}$ are assumed to 1.8, 1.6, and 1.4. By Snell's law, the respective critical angles of refraction $\left(\theta_{\mathrm{c}}\right)$ for $l$ - and $r-\mathrm{CP}$ light are evaluated to $51^{\circ}$ and $61^{\circ}$. The difference in the $\theta_{\mathrm{c}}$ angles of $l$ - and $r$-CP light, thus, efficiently acts as chiroptical filter between $l$ - and $r$-CP light at the colloid-liquid interface, such as a quarter-wave plate toward unpolarized light.

For clarity and visibility of the WGM concept, we demonstrate how an incident light travels interior of the $\mu \mathrm{m}$-size colloidal resonator using two macroscopic models consisting of a fused quartz cylindrical cuvette with optically smooth surfaces ( $22 \mathrm{~mm}$ in diameter, $1 \mathrm{~mm}$ cell wall in thickness) filled with a very dilute $\mathrm{CHCl}_{3}$ solution of stilbene 420 , which is a sky-blue-color luminophore, upon excitation of unpolarized light at $350 \mathrm{~nm}$. The cm-size cylindrical cuvette is a cross-section model of a $\mu \mathrm{m}$-size colloidal sphere shape. The macroscopic demonstration is a zoom-in model of light-matter interaction occurring in a $\mu \mathrm{m}$-scale resonator to persuade non-specialists.

From Figure $3 g$, the incident UV light at $\sim 0^{\circ}$ of the cuvette surface to excite stilbene 420 travels straightly and emits from the opposite side of the cuvette. In this case, only one-time interaction of light with the luminophore is possible. On the other hand, it is 
obvious from Figure $3 \mathrm{~h}$ that, when an incident light at a very specific angle of $\sim 50^{\circ}$ of the cuvette surface, the light reflects four times per one-cycle at the $\mathrm{CHCl}_{3}$-quartz interface and undergoes into the WGM with the traveling light circulation mode. This recycling enables the incident light to infinitely interact with the luminophore, if attenuation by the absorbing light is neglected. One can expect that endless light-matter interactions are possible, thus, causing significantly boosted chiroptical signals in the ground and photoexcited states.

When open-flow, $\mu \mathrm{m}$-size colloidal polymers with a smooth surface adaptable to the external physical and chemical biases are surrounded by an optofluidic medium, subtle chiral physical forces and chiral chemical sources are enhanceable by huge numbers of recycling times $(N)$ in the optofluidic colloidal polymers, in line with the scheme of WGM. This means that a subtle left-right imbalance interior of the colloid, e.g., $10^{-6} \%$ $e e$, is boostable by $10^{-6} \times \mathrm{N}$. This scenario is contrast to a conventional stereochemistry, photochemistry, and photophysics in homogeneous solution systems, in which the lightmatter interaction occurs one chance $(N=1)$ only. When aggregation-induced emission systems chiroptically fulfil the resonance condition, one can anticipate the significant enhancements in CPL and CD signals.

The recycling light should occur even at the $\mu \mathrm{m}$-size colloidal polymers that fulfil the resonant condition with relative RI values, between the polymers and fluidic medium. However, practically, the colloidal shape may not be an ideal sphere with an optically smooth surface, and an irregular shape with an optically rough surface diminishes this booster effect. The concept of WGM at the colloid-liquid interface, satisfying a resonance condition, along with specific RI values is applicable to boost chiral light-matter, lightchiral matter, and chiral light-chiral matter interactions.

To design the light recycling at the colloid-liquid interface, fine tuning RI at the specific wavelengths of the liquid medium is essential. It should be noted that RI values vary as a function of wavelength in a vacuum. This unique idea is valid for any optically opaque system, of chromophores and luminophores, in a heterogeneous medium, but is not applicable to optically transparent, homogeneous solutions. If the RIs between the colloid and surrounding medium are, by chance identical, any refraction, any reflection, and any scattering at the colloid-liquid interface do not occur. One can say that the colloidal mixture is optically transparent. The representative example is optically transparent pellet of poly(4-methyl-1-penetene (TPX ${ }^{\circledR}$, Mitsui Chemicals (Tokyo, Japan)). This transparency arises from the nearly identical RIs of crystalline and non-crystalline phases of TPX ${ }^{\circledR}$.

The traveling light speeds of $l$ - and $r$-CP in the colloidal polysilanes at $317 \mathrm{~nm}$ slow down to $1.67 \times 10^{8} \mathrm{~ms}^{-1}$ and $1.88 \times 10^{8} \mathrm{~ms}^{-1}$, respectively. However, the wavelengths of the incident $l$ - and $r$-CP light in a vacuum shorten to 176 and $198 \mathrm{~nm}$ in the colloid, respectively. As a result, the incident $317 \mathrm{~nm} l-\mathrm{CP}$ light results in a greatly shortened $l$-CP light of $176 \mathrm{~nm}$. Similarly, the incident $317 \mathrm{~nm} r$-CP light becomes a slightly shortened light of $198 \mathrm{~nm}$. If the $176 \mathrm{~nm}$ slow-down $l$-CP light was employed, multiple total internal reflections occurred efficiently (for example, 12 times) in the colloids compared to that of the slow-down $198 \mathrm{~nm} r$-CP light. Increasing the number of total internal reflection (TIR) of CP light at the colloid-liquid interface meets the greater opportunity of chiral light-matter interactions. The recurring multiple TIR process at the colloid-liquid interface is an important step.

The slow-down $317 \mathrm{~nm} l$-CP light relaxes to the $330 \mathrm{~nm} r$-CP light along with a change in CD sign within the colloids. The faster $317 \mathrm{~nm} r$-CP light migrates to the $330 \mathrm{~nm} r$-CP light without significant change in the CD sign. Owing to a great suppression of the 325 $\mathrm{nm} l$-CP light, the slow-down $330 \mathrm{~nm} r$-CP light emits dominantly from the $S_{1}$ state with an apparent minimal Stokes' shift. This explanation is verified by the ORD spectrum in the fluidic medium with the tuned $n_{\mathrm{D}}$ (Figure $3 \mathrm{c}$ ). The ORD spectrum, as a function of the incident wavelength of $l$ - and $r$-CP light, can detect differences in traveling light speed between $l$ - and $r$-CP in an optically active colloidal particle. 


\subsection{Controlled Chirogenesis from Optically Inactive Helical Polysilanes Endowed with Limonene Chirality}

Optically active, colloidal helical polysilanes are instantly generated by solely adding poor solvent to a homogeneous solution of optically active helical polysilanes carrying chiral substituents [219]. This approach is, however, inevitably needed to use expensive chiral biomolecular and artificial sources and multiple synthetic steps when we intend to introduce chiral substituents to Si-Si backbone [219-223]. Herein, we showcase a facile, inexpensive, environmentally-friendly approach to instantly generate CIE-CPL and CIE-CD signals of colloidal polysilanes from optically inactive helical polysilanes in a minute (Figure 4) [200,226]. Herein, the authors used artificial chiral alcohols and biomolecular chiral solvents for chirogenesis of colloidal $\sigma$ - and $\pi$-conjugated polymers (Figure 5) [198-207,212].
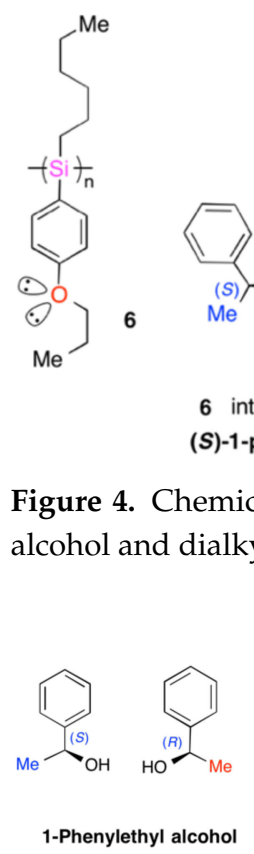
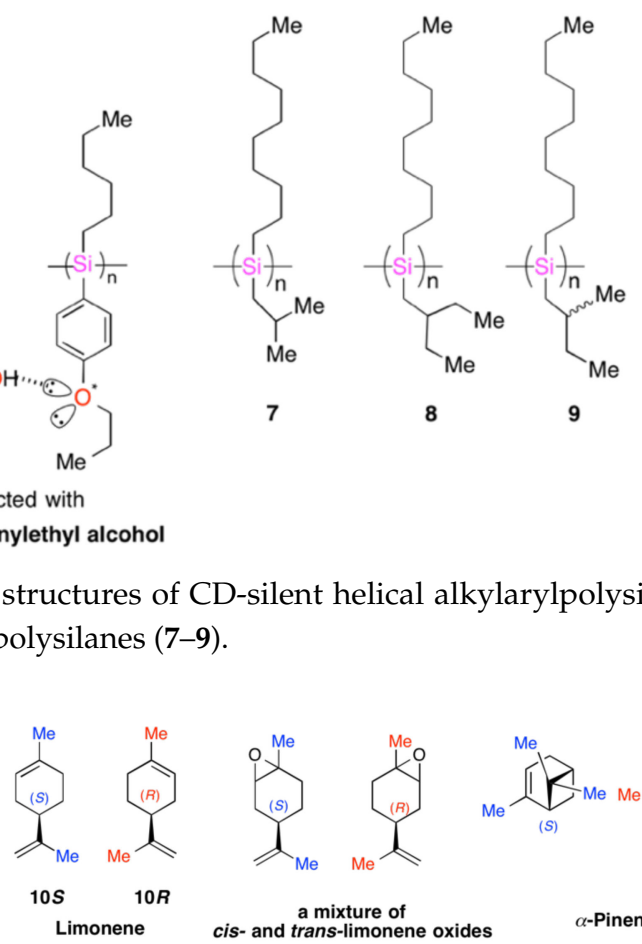

with

-1-phenylethyl alcohol

Figure 4. Chemical structures of CD-silent helical alkylarylpolysilane (6) with (S)-1-phenylethyl alcohol and dialkyl polysilanes (7-9).

Figure 5. Chemical structures of artificial chiral alcohols and biomolecular chiral solvents used for chirogenesis of colloidal polymers.

Previously, we reported that optically inactive colloidal helical 6 exhibits couplet-like CIE-CD by transferring molecular chirality of $(S)$ - and (R)-1-phenylethyl alcohols and other chiral alkyl alcohols when these chiral alcohols were used as solvent quantities [226]. It should be noted that $\mathbf{6}$ homogeneously dissolved in the alcohol solutions, adopting a CD-silent racemic helical state arising from a dynamic twisting between the left- and right-helices in a low energy barrier height in a double-well. By adding a poor solvent (methanol), CD-silent $\mathbf{5}$ generates CIE-CD effect from the colloidal $\mathbf{6}$ arising from mirror symmetry breaking (MSB). In this case, the resulting CD sign is determined solely by the chirality of alcohols used. It was driven by solvent molecules. The handed chiral $\mathrm{CH} / \mathrm{O}$ interaction is responsible for the CIE-CD (Figure 4) [226]. We, at that time, however, were not aware of the RI dependency of the chiral alcoholic solvents because any ideas of optofluidics, optical resonator, confinement of chiral light, and chiral optofluidics were lacking.

Our early studies prompted further experimentally tests on whether three CD-silent, rod-like, helical dialkyl polysilanes $(7,8,9)$ provide CIE-CD $(7,8,9)$ and CIE-CPL $(8,9)$ in the presence of inexpensive (S)-limonene (10S) and (R)-limonene (10R) [200]. We assumed that non-covalent attractive intermolecular interactions, such as chiral $\mathrm{CH} / \pi$, London 
dispersion, and van der Waals forces were possible to emerge CIE-CD and CIE-CPL signals. A precisely controlled RI of limonene-containing tersolvent was critical as well.

The CIE-CD and the corresponding UV spectra of the colloidal 7 and 8 are shown in Figure $6 \mathrm{a}, \mathrm{b}$. In the 7, dispersed in 10R-based tersolvent, the $g_{\mathrm{CD}}$ amplitudes at the bisignate $C D$ spectra boosted to +0.022 at $327 \mathrm{~nm}$ and -0.031 at $309 \mathrm{~nm}$, respectively [200]. As expected, the 7 in 10S-based tersolvent gave the mirror-symmetric $\mathrm{g}_{\mathrm{CD}}$ amplitudes with -0.021 at $327 \mathrm{~nm}$ and -0.033 at $309 \mathrm{~nm}$, respectively [200]. The CIE-CD values of the 7 are comparable to those of the 9 , but those in the 8 decreased by the magnitude of one-third. The bisigned $\mathrm{g}_{\mathrm{CD}}$ values of the 8 in $10 R$-based tersolvent were -0.007 at $331 \mathrm{~nm}$ and +0.010 at $317 \mathrm{~nm}$, respectively. Conversely, the 8 in 10S-based tersolvent gave the mirror-symmetrical $g_{\mathrm{CD}}$ values of +0.005 at $331 \mathrm{~nm}$ and -0.007 at $317 \mathrm{~nm}$, respectively. The degree of circular polarization of the 7,8 , and 9 was, however, not intense on the order of $1.0-1.5 \%$. The absolute magnitudes of the CIE-CD values of the 7,8 , and 9 are greatly dependent of $M_{\mathrm{w}}$. A representative $M_{\mathrm{w}}$ dependent $g_{\mathrm{CD}}$ values from the 7 is given in Figure 6c. The specific 7 with $M_{\mathrm{W}}=2.7 \times 10^{4}$ afforded the greatest $g_{\mathrm{CD}}$ value; 8 and $\mathbf{9}$ had similar $M_{\mathrm{W}}$-dependency of the CIE-CD effects [200].
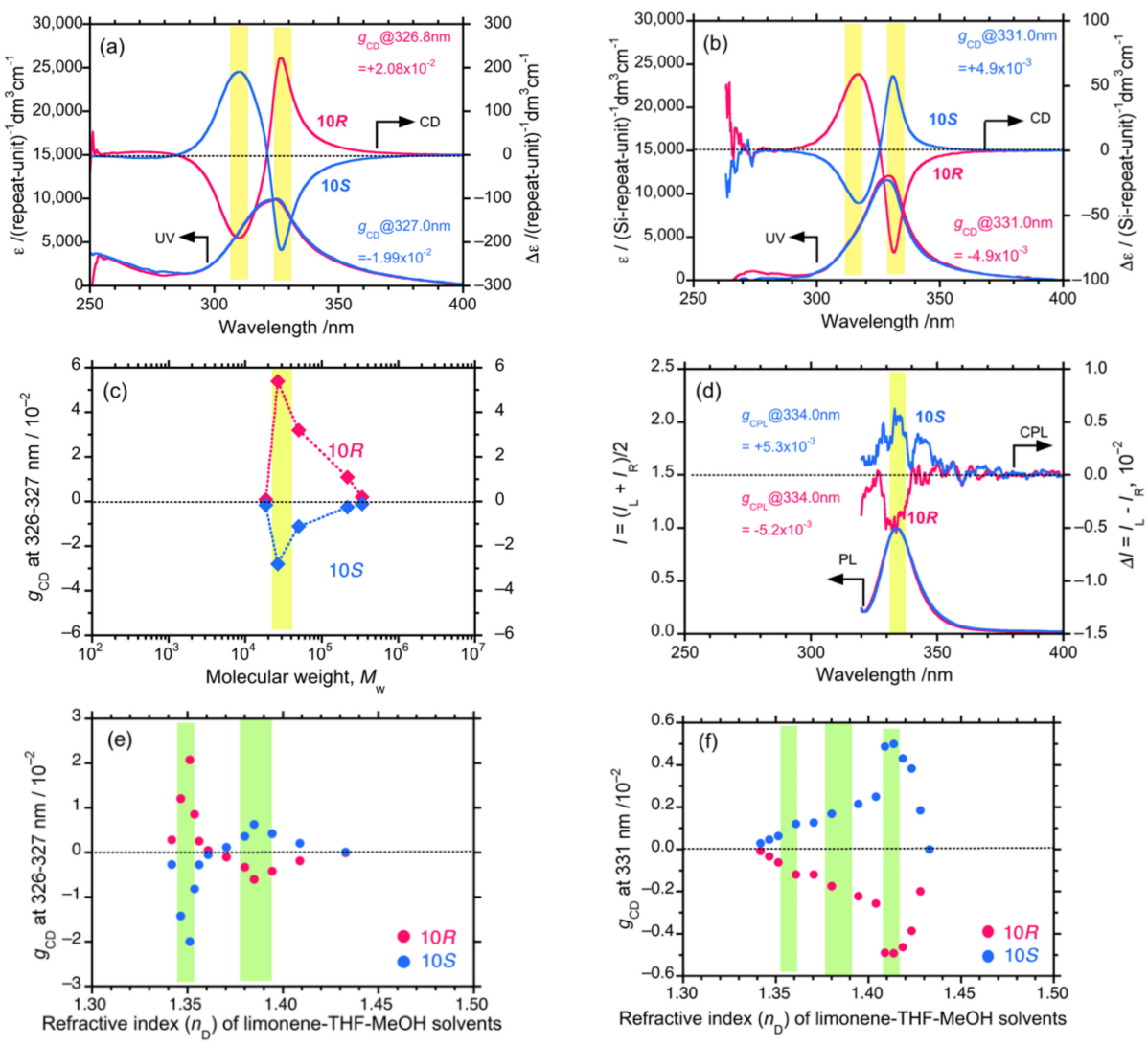

Figure 6. The CIE-CD and UV spectra from the colloidal: (a) 7 and (b) 8. (c) The $g_{C D}$ of the 7 as a function of weight average molecular weight of polymer $\left(M_{\mathrm{w}}\right)$ of the 7. (d) CIE-CPL and PL spectra of the 8. The $g_{\mathrm{CD}}$ of the: (e) 7; and (f) 8 as a function of refractive index at Na-D line $\left(n_{\mathrm{D}}\right)$ in the limonene containing tersolvent. Modified from an original article [200] and the book chapter [212].

Among the 7, 8, and 9 showing weaker $g_{\mathrm{CD}}$ values, only the 8 and 9 revealed weaker CIE-CPL associated with the absolute value of $g_{\mathrm{CPL}},\left|g_{\mathrm{CPL}}\right| \approx 0.005$ (Figure 6d) [200]. The 7 did not emit any PL due to unresolved reasons, thereby, revealed no detectable CPL signals. From Figure $6 a, b$, the CD sign of the 7 is opposite of that of the 8 when the same limonene chirality was applied. However, the CD sign of the 7 inverted at the specific $n_{\mathrm{D}}=1.35$ of the $10 R$ - and 10S-based tersolvents (Figure 6e). The unchanged CD sign of the 8 showed an abrupt increment at $n_{\mathrm{D}}=1.41$ (Figure $6 \mathrm{f}$ ). Thus, subtle structural alterations 
in achiral alkyl side chains of helical polysilanes significantly affect the amplitudes and chiroptical sign of CD and CPL signals.

\subsection{Chirogenesis from Achiral $\pi$-Conjugated Polymers Endowed with Limonene Chirality}

From three CD-silent helical 7, 8, and 9 in homogeneous solution, we knew the CIE-CPL effects from the colloidal 8 and $\mathbf{9}$ and CIE-CD effect from the $\mathbf{7}, \mathbf{8}$, and $\mathbf{9}$ in the presence of $10 S$ and $10 R$ [200]. The results prompted to further investigate CIE-CD and CIE-CPL effects from achiral $\pi$-conjugated luminescent polymers in the presence of $\mathbf{1 0 S}$ and 10R and (S)- and (R)-pinene (Figure 5) [198,201-212]. Herein, the first successful limonene chirality transfer allowed for realizing CIE-CD and CIE-CPL effects from achiral poly [(9,9-dioctylfluorenyl-2,7-diyl)-alt-bithiophene] (11) [198] among several chain-like $\pi$-conjugated polymers (Figure 7) and hyperbranched poly(dioctylfluorene) [206]. The CIECD and CIE-CPL characteristics of 12 [203], 13 [202], 14 [204], 15 [198], 16 [198], 17 [205], 18 [205], 19 [201], and the hyperbranched polyfluorene [206] are nearly similar to those of 15. Moreover, the CIE-CD and CIE-CPL characteristics, including $g_{\mathrm{CD}}$ and $g_{\mathrm{CPL}}$ values in these colloidal polymers, were resonantly boosted at very specific RIs, associated with specific volume fractions in the limonene-based tersolvents.

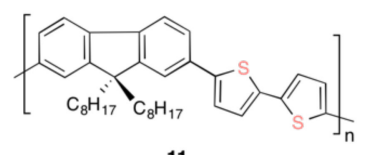

11
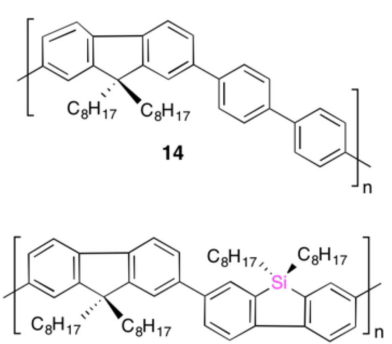

18
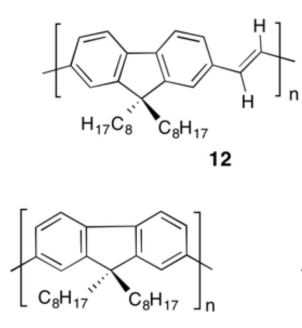

15

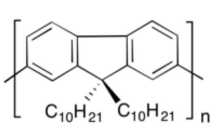

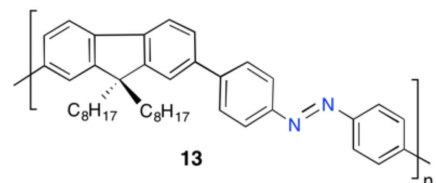



16

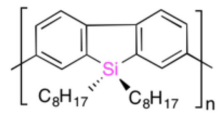

17

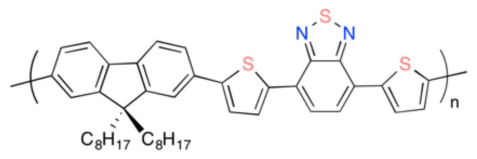

20

Figure 7. Chemical structures of optically inactive, achiral, and CD-silent $\pi$-conjugated polymers.

The UV-visible and PL spectra of $\mathbf{1 1}$ in homogeneous $\mathrm{CHCl}_{3}$ solution are given in Figure 8a. The PL spectrum is accompanied with three well-resolved vibronic signals at 500,534 , and $578 \mathrm{~nm}$ with a spacing of $\approx 1350 \mathrm{~cm}^{-1}$ [198]. Contrarily, the corresponding UV-visible spectrum is structureless broad bands at $\approx 457 \mathrm{~nm}$ (major) and $478 \mathrm{~nm}$ (a shoulder) [198]. This indicates that $\mathbf{1 1}$ in the photoexcited state adopts a highly ordered structure as a low entropy state. Conversely, $\mathbf{1 1}$ in the ground state adopts a randomly fluctuated disordered structure as a high entropy state. The UV-visible and PL spectral characteristics in the homogeneous solution are representative of random coiled luminescent chromophoric polymers in dilute solutions [220-223]. The rotational freedom with an equal probability in twisting between left and right in $\mathrm{C}-\mathrm{C}, \mathrm{Si}-\mathrm{Si}$, and $\mathrm{Si}-\mathrm{C}$ bonds in the ground state results in the apparently broadened UV-visible absorption bands, leading to no detectable CD signals. The barrier heights of these single bonds are as small as $1-2 \mathrm{kcal} \mathrm{mol}^{-1}$ from our calculations $[198,201,203,220]$. 

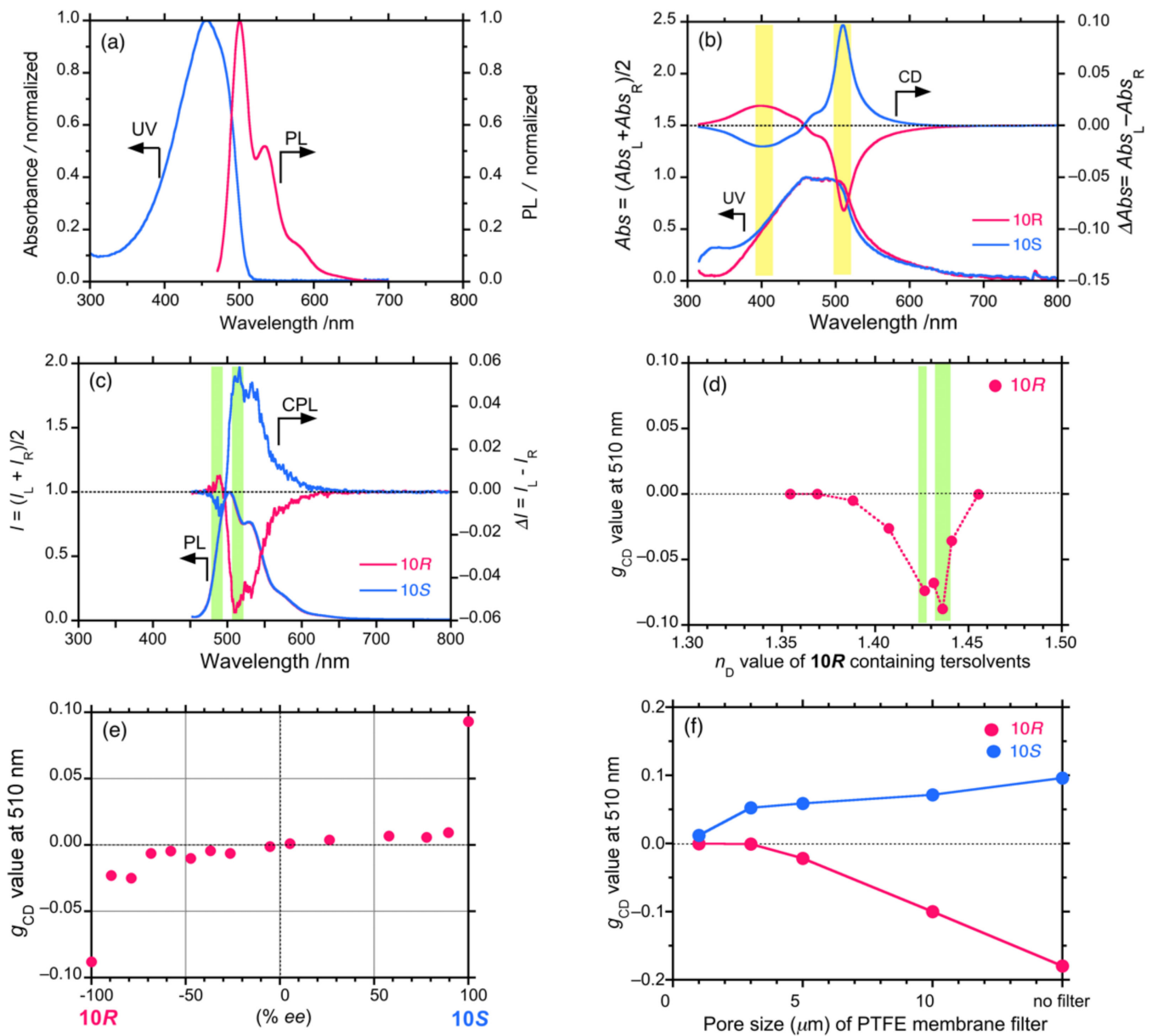

Figure 8. (a) UV-visible and PL spectra of $\mathbf{1 1}$ in a homogeneous $\mathrm{CHCl}_{3}$ solution; (b) $\mathrm{CIE}-\mathrm{CD}$ and UV spectra of the unfiltered colloidal 11 suspension in limonene- $\mathrm{CHCl}_{3}$-methanol tersolvent; (c) $\mathrm{CIE}-\mathrm{CPL}$ and PL spectra of the 11 in limonene- $\mathrm{CHCl}_{3}$ methanol tersolvent; (d) the $g_{\mathrm{CD}}$ value at $510 \mathrm{~nm}$ as the first Cotton band as a function of the $n_{\mathrm{D}}$ in the tersolvent; (e) The $g_{\mathrm{CD}}$ value of the unfiltered $\mathbf{1 1}$ as a function of the limonene enantiomeric excess (ee) value; (f) The $g_{\mathrm{CD}}$ value of the filtered $\mathbf{1 4}$ as a function of the colloid size. Modified from an original article [198] and the book chapter [212].

The colloidal $\mathbf{1 1}$ in the limonene-based tersolvents reveals nearly mirror-symmetrical CIE-CD and CIE-CPL spectra by the lack of rotational freedom in the ground and photoexcited states (Figure $8 b, c)$ [198]. The bisignate $g_{\mathrm{CD}}$ values are -0.085 at $510 \mathrm{~nm}$ and +0.042 at $394 \mathrm{~nm}$ (for 10R), respectively, and +0.114 at $510 \mathrm{~nm}$ and -0.041 at $394 \mathrm{~nm}$ (for 10S), respectively. Concurrently, the bisignate $g_{\mathrm{CPL}}$ values are evaluated to +0.012 at $489 \mathrm{~nm}$ and -0.058 at $511 \mathrm{~nm}(\mathbf{1 0 R})$, respectively, and -0.010 at $489 \mathrm{~nm}$ and +0.056 at $511 \mathrm{~nm}(\mathbf{1 0 S})$, respectively. In the $10 R$-based tersolvent, the $511 \mathrm{~nm}(-)$-CPL signal originates from the $510 \mathrm{~nm}-(-)-C D$ signal, while the $489 \mathrm{~nm}(+)-C P L$ band arises from the $394 \mathrm{~nm}(+)-C D$ signal. Likewise, the 10S-based tersolvent induces the opposite to those of the $\mathbf{1 1}$ led by the 10R-based tersolvent [88].

The $g_{\mathrm{CD}}$ value of the $\mathbf{1 1}$ is resonantly boosted at $n_{\mathrm{D}}=1.44$ of the limonene-containing tersolvent (Figure $8 \mathrm{~d}$ ). Notably, the $g_{\mathrm{CD}}$ value varies extraordinary in response to the ee value of limonene, exhibiting the so-called, negative cooperative effect (Figure 8e). The negative cooperativity indicates that enantiomerically pure limonene chirality is needed to attain the highest $g_{\mathrm{CD}}$ value. Moreover, we are aware that the $g_{\mathrm{CD}}$ value increases as the colloidal size increases (Figure 8f) [198]. A larger size of the colloidal particles fulfils morphology-dependent resonance condition in the WGM-origin chiroptical resonators known as cavity quality, indicated by theories $[227,228]$ and experiments $[229,230]$. 
4.5. Fully Controlled Absolute Photochirogenesis from Achiral $\pi$-Conjugated Polymers Endowed with Excitation Wavelength Dependent Circularly Polarized Light Chirality

The colloids exhibiting significantly boosted CIE-CD and CIE-CPL signals are instantly generated at room temperature with help from limonene with $(S)$ - and $(R)$-chirality from optically inactive $\sigma-/ \pi$-conjugated polymers. The $g_{\mathrm{CD}}$ and $g_{\mathrm{CPL}}$ signals are resonantly boosted at the specific RI of the optofluidic media. These results encourage us to further investigate a possibility of AAS with light or APC from two optically inactive colloidal 13 [55] and 11 [54] by dispersing to achiral solvents with a very specific RI endowed with CP light chirality (Figure 9). Recently, we demonstrated that the controlled APC with $\mathrm{CP}$ light (CAPC) from achiral colloidal polymethacrylate carrying azobenzene 21 in a tuned achiral cosolvent is possible by $\mathrm{CP}$ light, with wavelength and sense as purely chiral physical force [56] (Figure 8). We had confidence that, in the colloidal systems of 11, 13, and 21, a hand of CP light is not a deterministic factor for their product chirality and that both a hand and an excitation wavelength of CP light are subject for the CAPC with CP light.
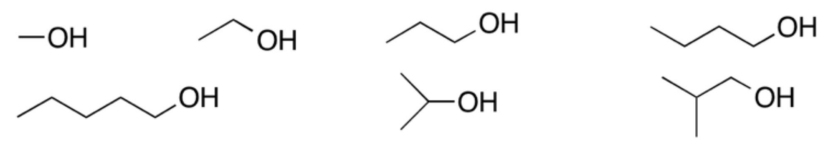

Figure 9. Chemical structures of alcohols used for $l$ - and $r$-CP light controlled absolute photochirogenesis (CAPC) experiments for the colloidal 11 and 13.

4.5.1. Achiral $\pi$-Conjugated Polymer Containing Azobenzene Unit as a Backbone Endowed with Circularly Polarized Light Chirality

In this section, we demonstrate that CAPC operated in the RI-tuned optofluidic medium is efficiently realized when wavelength-controlled $r$ - and $l$-CP light is employed as chiral electromagnetic force. The CAPC conducted by achiral optofluidic medium enables all chiroptical polarization (chirogenesis), depolarization (racemization or chiroptical erase), inversion (anti-chirogenesis), retention (memory), and switching in the $\mu \mathrm{m}$-size colloidal 11, 13, and 21 (see, later Section 4.5.3), as evidenced by their CIE-CD and CIE-CPL spectral characteristics.

As mentioned in Section 1.2.1, LeBel and 1894, van't Hoff proposed AAS with $r$ - and l-CP light. Kuhn and Broun proved their idea as a photodestruction mode of AAS [46]. Their works prompted researchers to catalyze AAS study for a century because expensive chiral chemical substances are no longer needed [47-49,231-233]. Possibly, most researchers might concur that $l$-CP light produces left-hand (or right-hand) molecules preferentially, or vice versa, because the product chirality is determined solely by the hand of CP light. The author was one of the researchers.

In Figure 10a,b, the CIE-CD, CIE-ORD, and UV spectra of the colloidal 13 are displayed when $r$ - and $l$-CP light source is excited at $436 \mathrm{~nm}$ [54]. The bisignate $g_{\mathrm{CD}}$ values at the first and second Cotton bands are -0.025 at $500 \mathrm{~nm}$ and +0.021 at $367 \mathrm{~nm}$ for $r$-CP light, respectively [54]. Conversely, they are +0.025 at $509 \mathrm{~nm}$ and -0.027 at $364 \mathrm{~nm}$ for $l-C P$ light, respectively. The 13 with negative-couplet CIE-CD upon excitation of $r$-CP light at $436 \mathrm{~nm}$ results in nearly zero CIE-CD signals upon excitation of $l$-CP light at $436 \mathrm{~nm}$ for 5-10 min (Figure 10c). A prolonged excitation of $l-C P$ light for $51 \mathrm{~min}$ led to an ideal, mirror-symmetric, positive-couplet CIE-CD signal (Figure 10c). An alternative excitation between $r$ - and $l$-CP light sources enables chiroptical inversion and switching between the positive- and negative-couplet CIE-CD signals [54]. It should be noted that $\mathbf{1 3}$ and unsubstituted azobenzene dissolved in dilute homogeneous $\mathrm{CHCl}_{3}$ solutions are weakly emissive. The 13, however, does not reveal any detectable CIE-CPL and PL spectra due to aggregation-caused quenching mechanism [187]. 

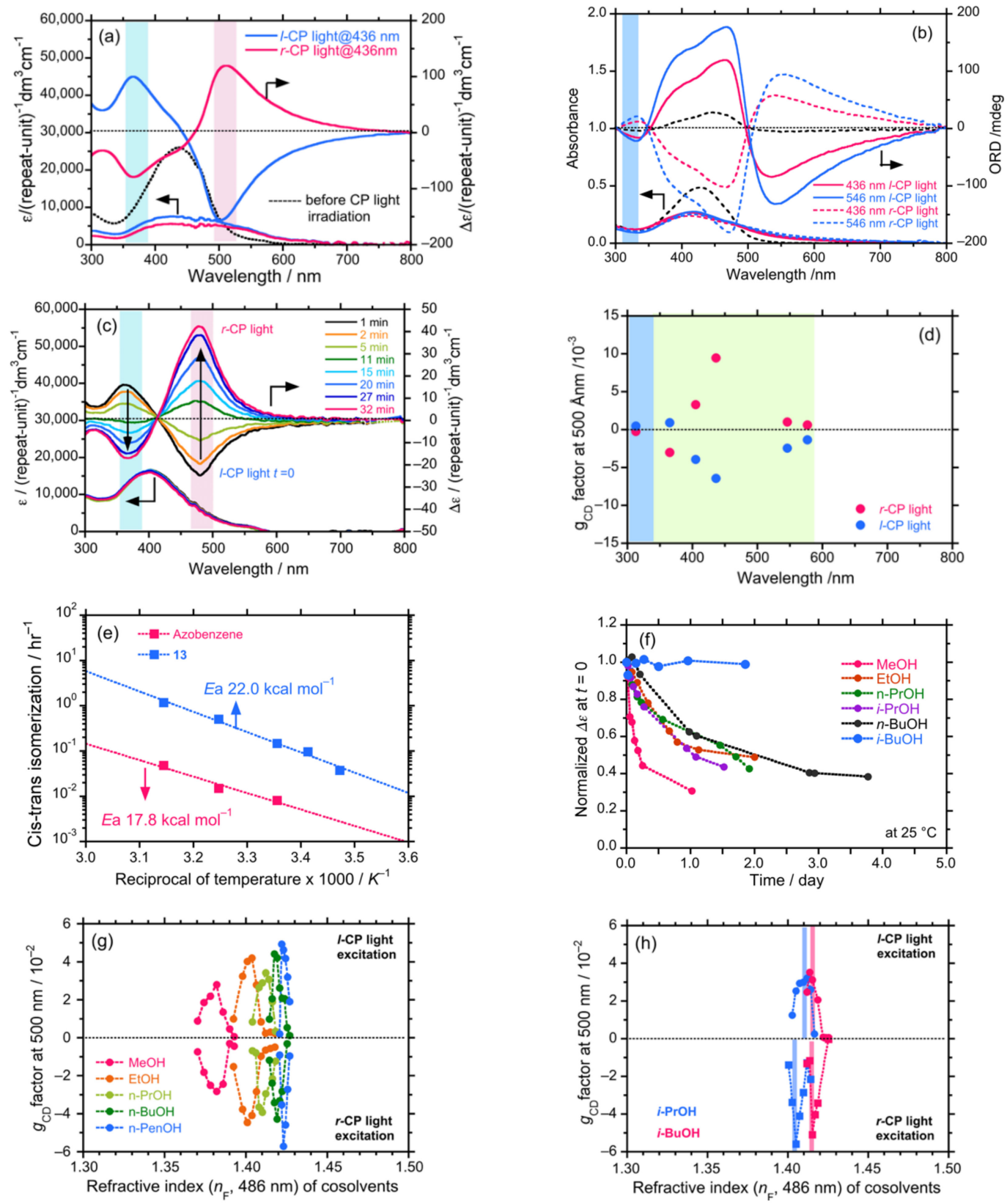

Figure 10. (a) CIE-CD and UV spectra of the colloidal 13 upon excitation with $r$ - and $l$-CP light at $436 \mathrm{~nm}$; (b) CIE-CD of the 13 initially generated by $l-C P$ light at $436 \mathrm{~nm}$, followed by $l-C P$ light excitation at $436 \mathrm{~nm}$; (c) The Arrhenius plots of the 13 and unsubstituted azobenzene during cis-trans thermal isomerization; (d) Alcohol-dependent thermal chiroptical stability of the CP-light source induced the colloidal cis-13 at $25{ }^{\circ} \mathrm{C}$. The $g_{\mathrm{CD}}$ values as a function of the $n_{\mathrm{F}}$ values of (e) non-branched alcohol- $\mathrm{CHCl}_{3}$ cosolvents and (f) isoalcohol- $\mathrm{CHCl}_{3}$ cosolvents. Modified from an original article [54] and the book chapter [212].

We are aware of an anomaly of an apparent chiroptical inversion for the product chirality of 13 when excited at $313 \mathrm{~nm}$ with the same $l$-CP light source; the positive-sign, weak CD signals excited at $313 \mathrm{~nm}$ only, while the negative-sign, intense CD signals upon excited at $365 \mathrm{~nm}, 405 \mathrm{~nm}, 436 \mathrm{~nm}, 549 \mathrm{~nm}$, and $577 \mathrm{~nm}$ (Figure 10d). Likewise, under the same $r$-CP light excitation, the product chirality at $313 \mathrm{~nm}$ is opposite to that at $365 \mathrm{~nm}$, $405 \mathrm{~nm}, 436 \mathrm{~nm}, 549 \mathrm{~nm}$, and $577 \mathrm{~nm}$.

The Arrhenius plots of the colloidal $\mathbf{1 3}$ and unsubstituted azobenzene during cis-trans thermal isomerization indicates that the activation energy $\left(E_{\mathrm{a}}\right)$ from cis-13 to trans-13 in a $\mathrm{CHCl}_{3}$-methanol cosolvent is $E_{\mathrm{a}} \approx 22 \mathrm{kcal} \mathrm{mol}^{-1}$ [54,121], which is slightly higher than 
that of the unsubstituted azobenzene of $E_{\mathrm{a}} \approx 18 \mathrm{kcal} \mathrm{mol}^{-1}$ (Figure 10e). The higher $E_{\mathrm{a}}$ value of the 13 contributes to the long-term chiroptical retention memory at ambient temperature. Likewise, from the Eyring plot that indicates the activation enthalpy $\left(\Delta H^{\ddagger}\right)$-activation entropy $\left(\Delta S^{\ddagger}\right)$ relationship, the thermally excited cis-trans isomerization of azobenzene moieties in the $\mathbf{1 3}$ should obey the rotation mechanism rather than the inversion one $[54,234]$. The rotation mechanism arises from restricted rotational freedom of azobenzene moieties in the colloids. Thus, the chirality of $r$ - and $l$-CP light can efficiently drive all of the chiroptical modes; generation, inversion, erase, switching, and short-term/long-term memory.

The thermal chiroptical stability of the $\mathbf{1 3}$ largely depends on the structure of the alcoholic solvents used (Figure 9). The $g_{C D}$ value of the $\mathbf{1 3}$ in methanol gives the shortest lifetime of $4-5 \mathrm{~h}$. The $g_{\mathrm{CD}}$ values in other non-branched alcohols and isopropanol diminishes in two days (Figure 10f). Notably, the $g_{\mathrm{CD}}$ value in isobutanol remains unchanged for at least two days. The proper choice of alcohols allows for the efficient generation and desired retention times of the chiroptical properties.

In the $\mathbf{1 3}$ systems, we re-emphasize that the precisely controlled RI in the alcohol$\mathrm{CHCl}_{3}$ cosolvents is key for the CIE-CD signals disregard of the non-branched and branched alcohols (Figure $10 \mathrm{~g}, \mathrm{~h}$ ). Using either $r$ - or $\mathrm{l}$-CP light as a chiral electromagnetic force, the absolute $g_{\mathrm{CD}}$ values, $\left|g_{\mathrm{CD}}\right|$, of the $\mathbf{1 3}$ are resonantly enhanced at a specific $n_{\mathrm{F}}$ value-that is, RI at $486.1 \mathrm{~nm} ; n_{\mathrm{F}}=1.382$ for methanol, 1.404 for ethanol, $1.410-1.412$ for $n$-propanol, 1.418 for $n$-butanol, 1.426 for $n$-pentanol, 1.405-1.411 for isopropanol, and 1.415 for isobutanol.

4.5.2. Achiral Luminescent $\pi$-Conjugated Polymer Endowed with Excitation Wavelength Dependent Circularly Polarized Light Chirality

The knowledge of the non-emissive colloidal 13 with azobenzene moiety led us to design the CAPC of the colloidal polymer made of highly luminescent 11 lacking photochromic moieties upon excitation of $r$ - and $l$-CP light sources at six different wavelengths (313 nm, $365 \mathrm{~nm}, 405 \mathrm{~nm}, 436 \mathrm{~nm}, 549 \mathrm{~nm}$, and $577 \mathrm{~nm}$ ). The CAPC behaviors are readily characterized by the CIE-CPL and CIE-CD spectra.

Figure 11a,b displays the CIE-CD and UV spectra of the colloidal $\mathbf{1 1}$ by exciting the two different $l$-CP light sources at 546 and $365 \mathrm{~nm}$ and by the two different $r$-CP light sources at 546 and $365 \mathrm{~nm}$, respectively. Unexpectedly, it is obvious that the positive-couplet CD spectrum endowed with the $546 \mathrm{~nm}$ l-CP light source inverts to negative-couplet CD of the $365 \mathrm{~nm}$ l-CP light source. Likewise, the negative-couplet CD spectrum induced by the $546 \mathrm{~nm} r$-CP light source becomes positive-couplet CD when the $365 \mathrm{~nm} r$-CP light source is applied. An excitation of CP light at 313 and $405 \mathrm{~nm}$ shows a similar tendency as the $365 \mathrm{~nm}$ excitation, while an excitation of CP light at $436 \mathrm{~nm}$ and $577 \mathrm{~nm}$ reveals a similar tendency as the $546 \mathrm{~nm}$ excitation. Upon excitation of the same $l$-CP (or $r-\mathrm{CP}$ ) light, the choice of shorter (UV) and longer (visible) wavelengths of CP light causes an inversion of chiroptical sign of the 11. Thus, we conclude that, the hand of CP-light, whether left or right, is not a deterministic factor for the CIE-CD sign of the 11.

Figure 11c displays a comparison between the CIE-CPL and PL spectra of the $\mathbf{1 1}$ upon excitation of the $546 \mathrm{~nm} l$ - and $r$-CP light sources. A positive couplet-like CPL spectrum led by the $546 \mathrm{~nm} r$-CP light source and negative-couplet-like CPL spectrum induced by the $546 \mathrm{~nm} l-\mathrm{CP}$ light source is obvious. The absolute magnitude of $g_{\mathrm{CPL}},\left|g_{\mathrm{CPL}}\right|$, is approximately $10^{-3}$. Upon excitation of $r$-CP light, the colloidal 11 reveals weak (+)-CPL at $570 \mathrm{~nm}$ arising from the $540 \mathrm{~nm}(+)-C D$ band, while the colloids showed a weak (-)-CPL signal at $518 \mathrm{~nm}$, originating from the $380 \mathrm{~nm}(-)$-broad CD signal. A tandem controlling hand and photoexcited wavelength of the CP light source allowed for the production of CPL-functioned 11 with $\phi=8 \%$ and $\left|g_{\mathrm{CPL}}\right|=(2-4) \times 10^{-3}$ at $540 \mathrm{~nm}$. The (+)-CIE-CD of the 11 generated by the $546 \mathrm{~nm} r$-CP light for $30 \mathrm{~nm}$ irradiation completely inverted to negative-couplets solely by the $54 \mathrm{~nm} l-\mathrm{CP}$ light for a prolonged irradiation of $120 \mathrm{~nm}$, as shown in Figure 11d. In other word, angular momentum $( \pm \hbar)$ of massless photon chirality enables to non-photochromic colloidal substances, resulting in induction and 
inversion of chemical chirality from achiral substances at ambient temperatures. Evidently, by optofluidically tuned RI of the achiral cosolvents, the CP light-driven CIE-CD signals of the 11 resonantly boosted at $n_{\mathrm{D}}=1.412$ disregard of $r$ - and $l$-CP light as excitation sources (Figure 11e).
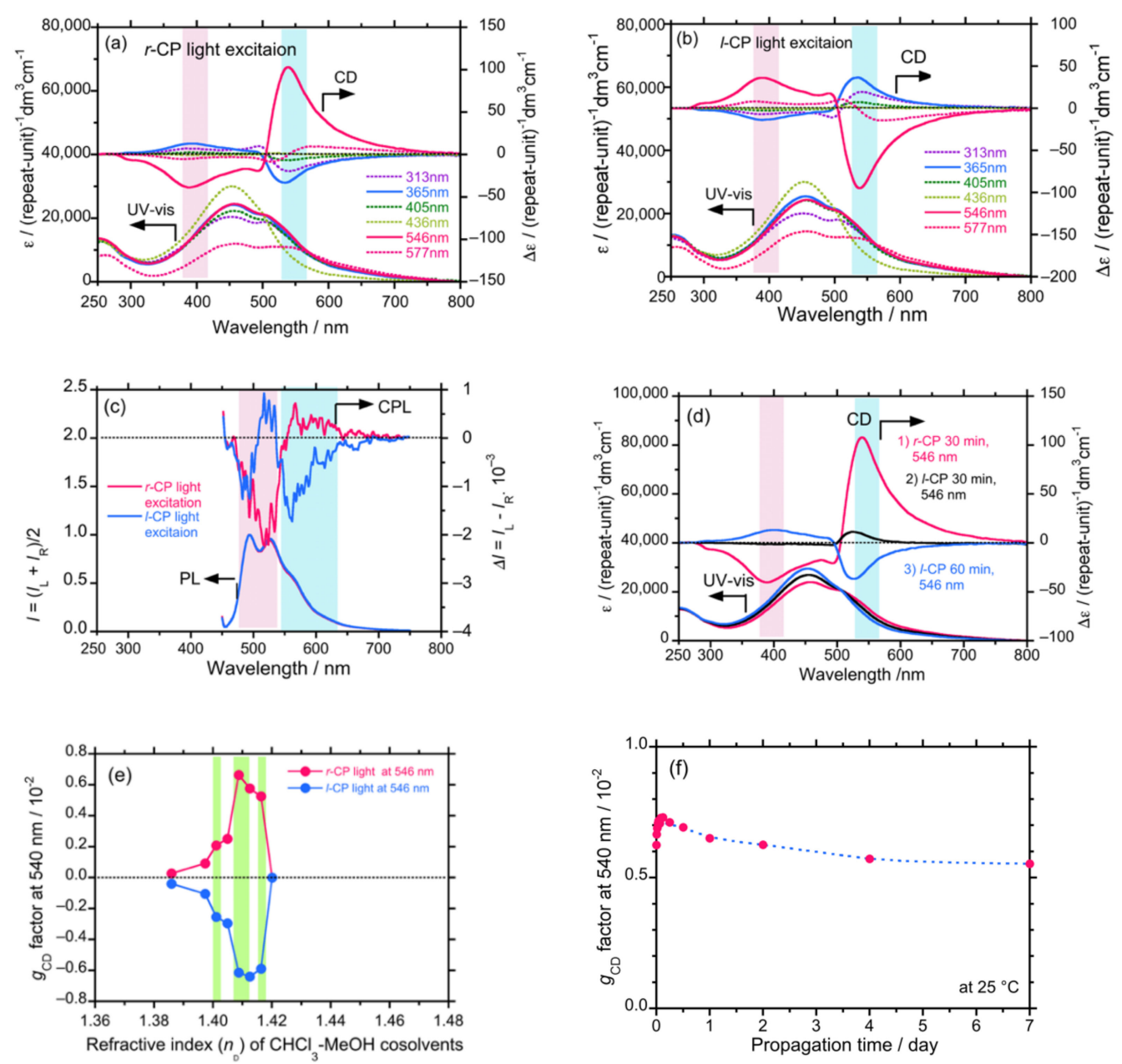

Figure 11. CIE-CD and UV spectra of the colloidal 11 in a $\mathrm{CHCl}_{3} / \mathrm{MeOH}$ cosolvent $(2.1 / 0.9$ (volume/volume) (v/v)) with $n_{\mathrm{D}}=1.41$ led by: (a) $r$-CP; and (b) $l$-CP light sources excited at six wavelengths ( $313 \mathrm{~nm}, 365 \mathrm{~nm}, 405 \mathrm{~nm}, 436 \mathrm{~nm}, 546 \mathrm{~nm}$, and $577 \mathrm{~nm}$ ); (c) CIE-CPL and PL spectra excited at $400 \mathrm{~nm}$ of the $\mathbf{1 1}$ led by the $546 \mathrm{~nm} r$-CP and $l-C P$ light sources for $60 \mathrm{~min}$; (d) chiroptical erase (racemization) and inversion (anti-chirogenesis) in CIE-CD associated with change in UV-visible spectra of the $\mathbf{1 1}$ conducted by the $546 \mathrm{~nm} r$-CP source for $30 \mathrm{~min}$, followed by $l$-CP light source for 30 and $60 \mathrm{~min}$; (e) the value of $g_{\mathrm{CD}}$ at $540 \mathrm{~nm}$ of the 11 conducted by the $546 \mathrm{~nm} r$-CP and $l$-CP light sources for $60 \mathrm{~min}$ as a function of $n_{\mathrm{D}}$ of $\mathrm{CHCl}_{3}-\mathrm{MeOH}$ cosolvents; (f) the chiroptical stability of the 11 generated by the $546 \mathrm{~nm} r$-CP light at $25^{\circ} \mathrm{C}$ in the dark. The $g_{\mathrm{CD}}$ value is plotted as a function of time. Modified from an original article [55] and the book chapter [212].

The CIE-CD spectra of the $\mathbf{1 1}$ are thermally stable and remain unchanged at $25{ }^{\circ} \mathrm{C}$ for at least seven days (Figure 11f). For comparison, non-colloidal $\mathbf{1 1}$ homogeneously dissolved in $\mathrm{CHCl}_{3}$ solution does not provide any detectable CD signals before and after prolonged irradiation of the $546 \mathrm{~nm} r$-CP light. The largely restricted rotational freedom associated with efficient confinement of the CP light source in the colloid as the optical resonator is essential in conducting CP light-driven CAPC experiments.

According to a theoretical study [235], chiroptical enhancement is possible when an ideal chiral sphere efficiently interacts with the surrounding chiral molecules. This means that the CIE-CPL and CIE-CD signals from the colloids are further enhanceable by tuning 
chiral fluidic medium. CP light is key in the migration and delocalization of photoexcited energy in optically active macro-colloids containing $\sim 10^{8}$ of chlorophyll upon excitation of unpolarized sunlight [171-174]. Note that chlorophylls contain three stereogenic centers at the peripheral positions of chlorophyll rings and two stereogenic centers in the alkyl chain tail.

4.5.3. Achiral Polymethacrylate Carrying Azobenzene Pendants Endowed with Excitation Wavelength Dependent Circularly Polarized Light Chirality

The concept of CAPC with light covers absolute asymmetric photosynthesis, photodestruction, and photoresolution. In the 1970s, Calvin et al. reported an anomaly of an excitation wavelength dependent CP light-driven photosynthesis-mode AAS recognized as switching product chirality of [8]-helicene in homogeneous toluene solution [52]. In 2014, Meinert et al. reported the wavelength dependent CP light-driven photodestruction-mode AAS revealing switching chirality when rac-alanine film is decomposed upon irradiation of two vacuum-UV light sources (184 and $200 \mathrm{~nm}$ ) [53]. Our results of CAPC from the $\mathbf{1 4}$ and the $\mathbf{1 1}$ as their colloidal states prompted to address an apt question, as to whether the excitation wavelength dependent switching chirality conducted by CP light-driven photoresolution mode AAS is generalizable; whether restricted rotational modes are crucial for CP-light driven CAPC [56].

We chose the colloidal 21 bearing achiral azobenzene moieties as pendants to test the excitation wavelength dependent $C P$ light-driven photoresolution mode AAS (Figure 12). The experiment of CAPC with light was designed to use three wavelengths $(313 \mathrm{~nm}, 365 \mathrm{~nm}, 436 \mathrm{~nm})$ for $r$ - and $l$-CP light sources to efficiently excite $\pi-\pi^{*}$ and/or $\mathrm{n}-\pi^{*}$ transitions of azobenzene moieties in restricted rotational states in the 21 . Contrarily, the azobenzene moieties are non-restricted, free rotational states in homogeneous solution. Similarly, restricted rotations along $C-C$ single bonds in the main chain of 21 are possible in the colloids, while non-restricted rotations of those $C-C$ single bonds occur in homogeneous solutions.

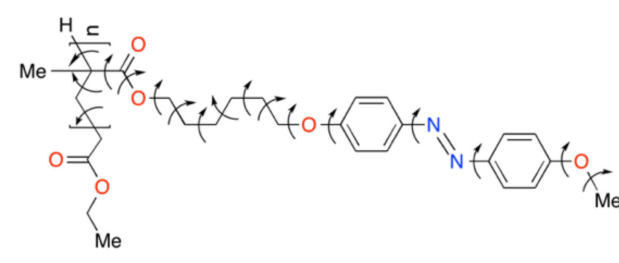

21

(restricted rotations in colloidal pariticle in a mixed $\mathrm{MeOH}$ and 1,2-dichloroethane)

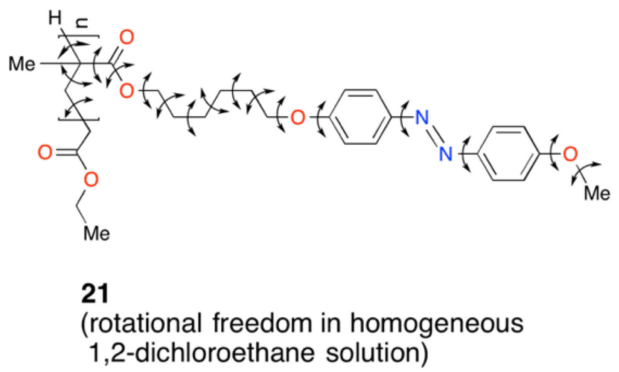

(rotational freedom in homogeneous
1,2 -dichloroethane solution)

Figure 12. Chemical structures of the colloidal 21 to employ wavelength-dependent CP light driven CAPC experiments. By tuning $n_{\mathrm{D}}$ value, a special mixture of 1,2-dichloroethane and methanol was used. Single-headed arrow means restricted rotation clockwise $(\mathrm{CW})$ or counter-clockwise (CCW), while double-headed arrow is possible to freely rotate CW and CCW.

In Figure 13a, we show the values of CD ellipticity at $313 \mathrm{~nm}$ (in mdeg, left ordinate) and $g_{\mathrm{CD}}$ at $313 \mathrm{~nm}$ (right ordinate) of the spherical shape colloidal 21 (125-300 nm in size) as a function of the $n_{\mathrm{D}}$ of 1,2-dichloroethane (DCE, good solvent, $n_{\mathrm{D}}=1.444$ ) and methylcyclohexane $\left(\mathrm{MCH}\right.$, poor solvent, $\left.n_{\mathrm{D}}=1.422\right)$ cosolvents upon excitation of very weak $l$ - and $r$-CP light sources at $365 \mathrm{~nm}$ for a short-period $\left(\approx 30 \mu \mathrm{W} \cdot \mathrm{cm}^{-2}, 60 \mathrm{~s}\right)$. Obviously, the values of CD ellipticity and $g_{\mathrm{CD}}$ steeply are maximized at a very specific $n_{\mathrm{D}}=1.425(\mathrm{DCE} / \mathrm{MCH}=$ $0.5 / 2.5$ (volume/volume) (v/v) regardless of the $365 \mathrm{~nm} l$ - and $r$-CP light sources. 

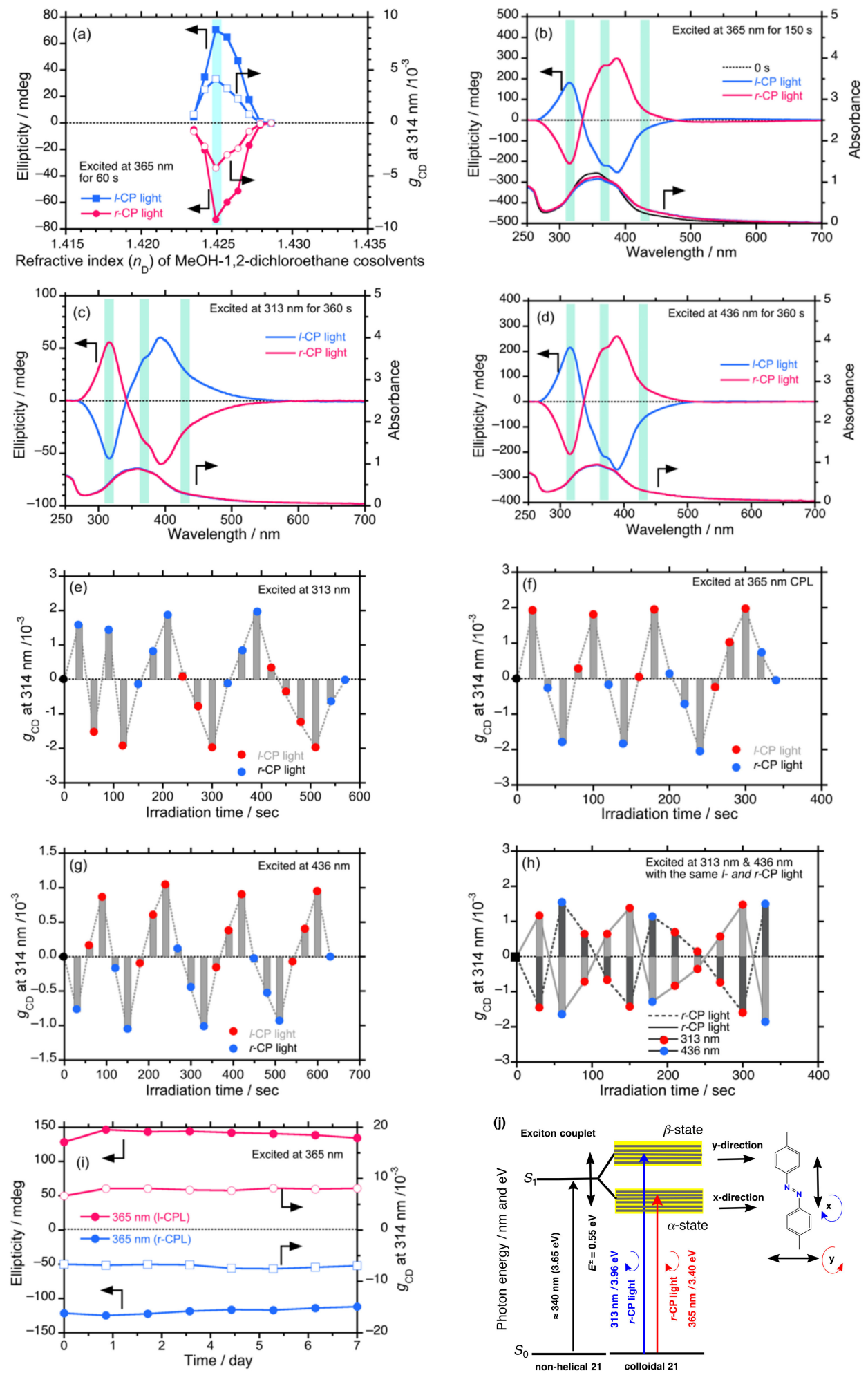

Figure 13. The CIE-CD and UV spectra of the colloidal 21 excited with: (a) $r$-CP light source at $546 \mathrm{~nm}$ and $l-C P$ light source at $365 \mathrm{~nm}$; and (b) $l$-CP light source at $546 \mathrm{~nm}$ and $l$-CP light source at $365 \mathrm{~nm}$; (c) CIE-CPL and PL spectra of the 21 excited with $r$ - and $l$-CP light source at $546 \mathrm{~nm}$; (d) the $g_{\mathrm{CD}}$ values as a function of $n_{\mathrm{D}}$ of the cosolvents. The alteration in the $g_{\mathrm{CD}}$ value at $314 \mathrm{~nm}$ of the 21 upon excitation with $r$ - and $l$-CP light sources (e) at $313 \mathrm{~nm}$; (f) at $365 \mathrm{~nm}$; (g) at $436 \mathrm{~nm}$; and (h) at $313 \mathrm{~nm}$ and $436 \mathrm{~nm}$; (i) Thermal stability of the 14. The $g_{\mathrm{CD}}$ value as a function of solvent temperature; (j) Anti-Kasha's rule and Jablonski diagram of the colloidal 21. Modified from the original article [56]. 
At the specific $n_{\mathrm{D}}=1.425$ and volume fraction of DCE/MCH, we conducted $l$ - and $r$-CP light driven CAPC experiments of the colloid 21 at $365 \mathrm{~nm}(150 \mathrm{~s}), 436 \mathrm{~nm}(360 \mathrm{~s})$, and $313 \mathrm{~nm}(360 \mathrm{~s})$. From Figure 13b, the $365 \mathrm{~nm} r$-CP light source induces bisignate negative-couplet-like CD profile at $310 \mathrm{~nm}$ and $360-390 \mathrm{~nm}$, conversely, the $365 \mathrm{~nm} l-C P$ light induces bisignate positive-couplet-like CD profile. Likewise, the $436 \mathrm{~nm} r$-CP light source induces similar bisignate negative-couplet-like CD profile, as shown in Figure 13d.

Conversely, the $365 \mathrm{~nm} \mathrm{l-CP}$ and the $436 \mathrm{~nm} l-C P$ light sources induce bisignate positive-couplet-like CD spectra (Figure 13b,d). On the other hand, it is evident from Figure $13 \mathrm{c}$ that the $313 \mathrm{~nm} r-\mathrm{CP}$ and $l-\mathrm{CP}$ light sources induce bisignate positive and negative couplet-like CD profiles at $310 \mathrm{~nm}$ and $360-390 \mathrm{~nm}$, respectively. For comparison, the $436 \mathrm{~nm} r$-CP light-driven CAPC experiments to the colloids made of the starting monomer of $\mathbf{2 1}$ in MCH-DCE and $\mathbf{2 1}$ in homogeneous DCE solution did not induce any detectable CD signals [56].

These excitation-wavelength dependent CAPC experiments allow to verify a possibility of all chiroptical generation, erase, inversion, multiple switching, and a long-term memory characteristics of the $\mathbf{2 1}$ in the optofluidic medium with the $n_{\mathrm{D}}=1.425 \mathrm{using} l$ - and $r$-CP light sources at $313 \mathrm{~nm}$ (Figure 13e), $365 \mathrm{~nm}$ (Figure 13f), $436 \mathrm{~nm}$ (Figure 13g), and a tandem of $313 \mathrm{~nm}$ and $436 \mathrm{~nm}$ (Figure 13h). All of the chiroptical modes are possible when the $313 \mathrm{~nm}, 365 \mathrm{~nm}$, and $436 \mathrm{~nm} \mathrm{CP}$ light sources are employed disregard of $l$ - and $r-\mathrm{CP}$ light sources. The bisignate chiroptical sense of the 21 led by the $313 \mathrm{~nm} l$-CP light source is absolutely opposite to the sense induced by the $365 \mathrm{~nm}$ and $436 \mathrm{~nm} l$-CP light sources.

We re-confirmed that an alternative excitation of the $313 \mathrm{~nm} l$-CP and $436 \mathrm{~nm} l$-CP light sources to the 21 enables conducting all of the chiroptical modes. Likewise, the alternative excitation of the $313 \mathrm{~nm} r$-CP and $436 \mathrm{~nm} r$-CP light causes the opposite characteristics of the dual $l$-CP light sources. The resulting optically active 21 endowed with the $365 \mathrm{~nm} r$-CP and $l$-CP light sources had long-term memory effects in the dark for at least seven days (Figure 13i). This uniqueness arises from the very restricted rotations of the azobenzene moieties and the $\mathrm{C}-\mathrm{C}$ single bonds in the main chain of the $\mathbf{2 1}$ in the dark (Figure 12, left). The $\mathbf{1 6}$ in the ground state is a closed system that endures thermally activated energy at room temperature. Conversely, the $\mathbf{1 6}$ in the photoexcited state during CP-light irradiation works as the open-flow, soft-matter-based photonic resonator adaptable to the external $\mathrm{CP}$-light energy by confining into the resonator with the help of the tuned RI fluidic medium.

From the spectra shifts at the three major bands of the 21, which includes $v(\mathrm{C}-\mathrm{H})$ at $\sim 3000 \mathrm{~cm}^{-1}, v(\mathrm{C}=\mathrm{O})$ at $\sim 1730 \mathrm{~cm}^{-1}$, and $v(\mathrm{C}-\mathrm{O}-\mathrm{C})$ at $\sim 1250 / \sim 1150 \mathrm{~cm}^{-1}$, a prolonged photoirradiation with $r$-CP at $365 \mathrm{~nm}$ for 10 min leads the 21 with trans-azobenzene pendants to cis-azobenzene 21 colloids. As a result, the cis-colloidal 21 prevents an efficient $\pi-\pi$ stacking of azobenzene pendants, followed by production of an ill-organized, smaller size colloids due to a bent structure of cis-azobenzene. The cis-21 colloids in the cosolvents were transparent by the naked eye. The small size colloids, less than $\sim 100 \mathrm{~nm}$, appear inconvenient to efficiently confine a traveling light at the colloid-liquid interface and prevent the multiple reflection mode of WGM.

In Figure 13j, we propose a hypothesis of the anti-Kasha rule that, by combining exciton couplet theory, Kasha's rule, Jablonski diagram, and $x-y$ directions of azobenzene at the photoexcited state, two pathways as a spontaneous relaxation process from $\alpha$-state ( $y$-axis of azobenzene) with the lowest energy $(\sim 365 \mathrm{~nm})$ and the $\beta$-state ( $x$-axis of azobenzene) with higher energy $(\sim 313 \mathrm{~nm})$ are possible. The $r$-CP light sources at $365 \mathrm{~nm}$ and $313 \mathrm{~nm}$ would make $y$ - and $x$-axes of azobenzene twist $C C W$ and $C W$, respectively. Likewise, $l-C P$ light sources at 365 and $313 \mathrm{~nm}$ oppositely twist y- and x-axes of azobenzene CW and CCW, respectively [56]. The anti-Kasha rule is prominent to CP-light driven CAPC of colloidal 21, 11, and 14 in the RI-tuned optofluidic media. The anti-Kasha rule is applicable to characterize CIE-CD and CIE-CPL spectra in the controlled chirogenesis, as given in a Section 4.7. 


\subsection{Tempo-Spatial Chirogenesis}

4.6.1. Changes in Colloidal Sizes of Diaryl Polysilane with Propagation Time

In this section, we highlight two topics: (a) multiple resonance effects in the $g_{\mathrm{CD}}$ value of the colloidal 21 in the $10 R / \mathrm{CHCl}_{3} / \mathrm{MeOH}$ and $10 \mathrm{~S} / \mathrm{CHCl}_{3} / \mathrm{MeOH}$ tersolvents among the colloidal diaryl polysilanes 21-24 (Figure 14); and (b) tempo-spatial chirogenesis, including changes in colloidal sizes and CIE-CD signals at several volume fractions of $\mathbf{1 0 R}$ and $10 S$ in the tersolvents with propagation time [207].

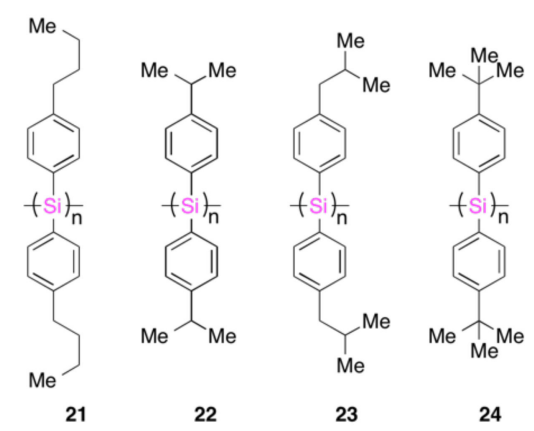

Figure 14. Chemical structures of CD-silent helical diaryl polysilanes revealing tempo-spatial CIE-CD effects with colloidal sizes in the limonene-containing optofluidic media.

According to the polymer consistent force-field (PCFF), diaryl polysilane $\mathbf{2 1}$ has four local minima from the potential energy surface due to phenyl-phenyl interaction (Figure 15a). Thus, 21 in a homogeneous solution reveals a CD-silent spectrum in the ground state (Figure 15b), and possibly a CPL-silent spectrum in the photoexcited state due to equal populations between two $P$-screw and two $M$-screw Si-Si backbones.

The $g_{\mathrm{CD}}$ values of the colloidal 21 show two and three extrema at the specific volume fractions of $\mathbf{1 0 R} / \mathrm{CHCl}_{3} / \mathrm{MeOH}$ and $10 \mathrm{~S} / \mathrm{CHCl}_{3} / \mathrm{MeOH}$ tersolvents (Figure 15e,f). This anomaly appears not unique for the 21 because a similar tendency in the $g_{\mathrm{CD}}-n_{\mathrm{D}}$ relations can be seen in the colloidal 14 (Figure 8d) and the colloidal 7 and 8 in the limonenecontaining tersolvents (Figure 6e,f). The $\mathbf{2 1}$ at the optimized volume fraction of $\mathbf{1 0 R}$ and $10 \mathrm{~S}$ in the tersolvents reveal clear bisignate CIE-CD spectra around $400 \mathrm{~nm}$ (Figure 15c) associated with mono-signate CIE-CPL spectra at $410 \mathrm{~nm}$ (Figure 15d). The (+)-sign CIECPL led by $10 R$ is identical to th (+)-sign of couplet-like CIE-CD at the first Cotton band of $\sim 408 \mathrm{~nm}$, conversely, the (-)-sign CIE-CPL with $10 \mathrm{~S}$ is the same of (-)-sign of CIE-CD with $10 S$ at $408 \mathrm{~nm}$. The relaxation scenario from the $S_{1}$ to $S_{0}$ states of the 21 obeys the Kasha rule $[216,217]$.

The reasons for two and/or three extrema in the $g_{\mathrm{CD}}$ values at the specific volume fractions of $\mathbf{1 0 R}$ and $\mathbf{1 0 S}$ is ascribed to three rotamers of $\mathbf{1 0 R}$ (and 10S) [236,237]. Nonrigid chiral monocyclic terpene $10 R$ consisting of isopropenyl group and cyclohexene ring has one freely rotable $\mathrm{C}-\mathrm{C}$ bond between the moieties. The rotable $\mathrm{C}-\mathrm{C}$ bond generates three equatorial rotamers of $10 R$ (equatorial 1 (Eq1), equatorial 2 (Eq2), equatorial 3 (Eq3), Figure 16) with nearly equal populations with different ORD spectra with different signs in the range of 200-589 nm (Eq1; 0.39 with an intense (+)-ORD, Eq2; 0.31 with an intense $(-)$-ORD, Eq3; 0.30 with a weak (-)-ORD) along with a small fraction of energetically unstable axial rotamers [236,237]. We assume that the packing structure of $\mathbf{2 1}$ in the colloids is reorganizing in response to a preferential rotamer of $5 R$ and $5 S$, whose fractions depend on a volume fraction of limonene in the tersolvents [207]. 

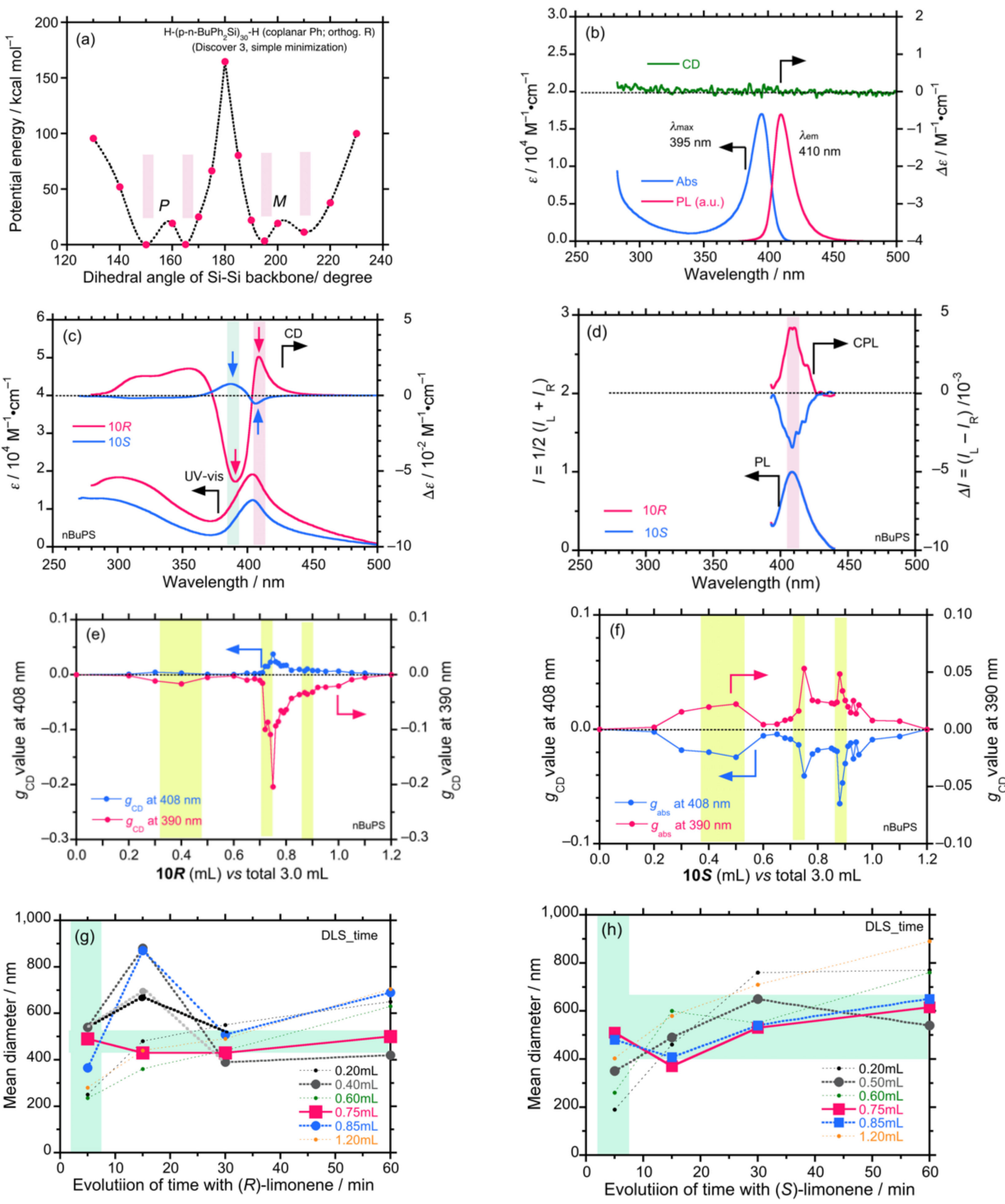

Figure 15. (a) Potential energy surface having four local minima of 21; (b) CD, UV-visible, and PL spectra of 21 in a dilute $\mathrm{CHCl}_{3}$ solution; (c) CIE-CD and UV-visible and (d) CIE-CPL and PL spectra of the colloidal 21 in the tersolvents of $10 R$ (or 10S) $/ \mathrm{CHCl}_{3} / \mathrm{MeOH}=0.75 / 0.30 / 1.95$ (volume/volume/volume) (v/v/v). The values of $g_{\mathrm{CD}}$ at $390 \mathrm{~m}$ and $408 \mathrm{~nm}$ of the 21 as a function of volume fraction of: (e) $10 R$ and (f) $10 S$ in the tersolvents of $10 R$ (or $10 S) / \mathrm{CHCl}_{3}(0.3 \mathrm{~mL}) / \mathrm{MeOH}$ and total volume $3.0 \mathrm{~mL}$. The colloidal sizes of $\mathbf{2 1}$ at several volume fraction of: (g) $10 R$ and (h) $10 \mathrm{~S}$ in the tersolvents as a function of propagation time. Modified from an original article [207]. 




Figure 16. Three equatorial rotamers (equatorial 1 (Eq1), equatorial 2 (Eq2), equatorial 3 (Eq3)) of 10R at two local and one global minima optimized with Gaussian09 (DFT, B3LYP, 6-31G(d,p) basis set), suggested by two papers [236,237]. The calculated optical rotations at $365 \mathrm{~nm}$ with a relative population of Eq1, Eq2, Eq3 are $+1501^{\circ}(0.39),-428^{\circ}(0.31)$, and $-206^{\circ}(0.30)$, respectively [237]. The other calculated populations of Eq1, Eq2, Eq3, and total of three axial rotamers are 0.32, $0.21,0.43$, and 0.04 , respectively [236].

In Figure 15g,h, the tempo-spatial behaviors in the colloidal size of $\mathbf{2 1}$ at several volume fractions of $10 R$ and $10 S$ in their tersolvents are given by means of dynamic light scattering measurements. We can see the marked alteration in the colloidal sizes (200-900 nm) of $\mathbf{2 1}$ in the $\mathbf{1 0 R}$ and $\mathbf{1 0 S}$ tersolvents; the colloid sizes at two specific volume fractions ( 0.40 and $0.75 \mathrm{~mL}$ for $10 R ; 0.75$ and $0.85 \mathrm{~mL}$ for $10 S)$ appear unchanged with time, while those at other volume fractions seem to fluctuate and/or grow gradually with time. The resonant volume fractions in Figure 15e,f) are possibly connected to the tempo-spatial behaviors of the colloidal sizes, followed by the maximizing $g_{\mathrm{CD}}$ values.

4.6.2. Time-Dependent Evolution of CIE-CD and CIE-CPL in Co-Colloids of Achiral $\pi$-Conjugated Polymer and Helical Dialkyl Polysilanes

Based on the tempo-spatial behaviors of the colloidal 21 endowed with $10 R$ and 10S, we designed co-colloids comprising achiral $\pi$-conjugated 20 (Figure 7) and rod-like helical dialkyl polysilanes, $\mathbf{5} S$ and $\mathbf{5 R}$ (Figure 2). The optimized molar ratio as repeating units of $\mathbf{2 0}$ and $\mathbf{5 R}$ (or $5 S$ ) is found to be 1:1 (Figure 16h). The optimized $n_{\mathrm{D}}$ value in the $\mathrm{CHCl}_{3}-\mathrm{MeOH}$ cosolvents enabling the largest RI value of the co-colloid is found to be $n_{\mathrm{D}}=1.405$ in a cosolvent of $\mathrm{CHCl}_{3} / \mathrm{MeOH}=1 / 2(\mathrm{v} / \mathrm{v})$ (Figure 17h). Note that the rigid rod-like helical $5 R$ and $5 S$ act as helical scaffolding to non-helical $\pi$-conjugated polymers.

The alterations of the CIE-CD/UV-visible-NIR spectra and the CIE-CPL/PL spectra of the 1:1 co-colloids of $\mathbf{2 0}$ and $\mathbf{5} \boldsymbol{R}$ as a function of propagation time are displayed in Figure $17 \mathrm{a}, \mathrm{b}$. Likewise, the changes in the CIE-CD/UV-visible-NIR spectra and the CIE-CPL/PL spectra of the 1:1 co-colloids of 20 and $5 S$ with propagation time are given in Figure 17a,b. Obviously, the absolute magnitudes in CIE-CD and CIE-CPL increase and tend to level-off for a prolonged time of $24 \mathrm{~h}$, regardless of $5 R$ and $5 S$. The $20 / 5 S$ and $20 / 5 R$ co-colloids generate CD-activity and CPL-activity from optically inactive $\mathbf{2 0}$ with help from main chain helicity and/or side chain chirality of $5 S$ and $5 R$. Time-dependent reorganization of 20 , with help from $5 R / 5 S$ in fluidic media occurs on the order of hours at room temperature.

To associate the $g_{\mathrm{CD}} / g_{\mathrm{CPL}}$ values with the colloidal size, we characterized the timedependent co-colloidal sizes using a dynamic light scattering (DLS) method, as shown in Figure 17f,g. An approximately $650 \mathrm{~nm}$ size of the 20-5S co-colloids progressively reach $\sim 2000 \mathrm{~nm}$ within $24 \mathrm{~h}$ (Figure 17f). Likewise, an approximately $400 \mathrm{~nm}$ size of the 20-5R co-colloids in the beginning attains $\sim 1800 \mathrm{~nm}$ within $24 \mathrm{~h}$ (Figure 17e). The larger sizes of the co-colloids after prolonged propagation times are responsible for the increases in the larger $g_{\mathrm{CD}}$ and $g_{\mathrm{CPL}}$ values of the 20-5R and 20-5S co-colloids associated with a significant red-shift in CIE-CD and CIE-CPL spectra. It takes several hours to re-organize 20 with help from the Si-Si main chain helicity and/or side chain chirality of $5 R$ and $5 S$. 

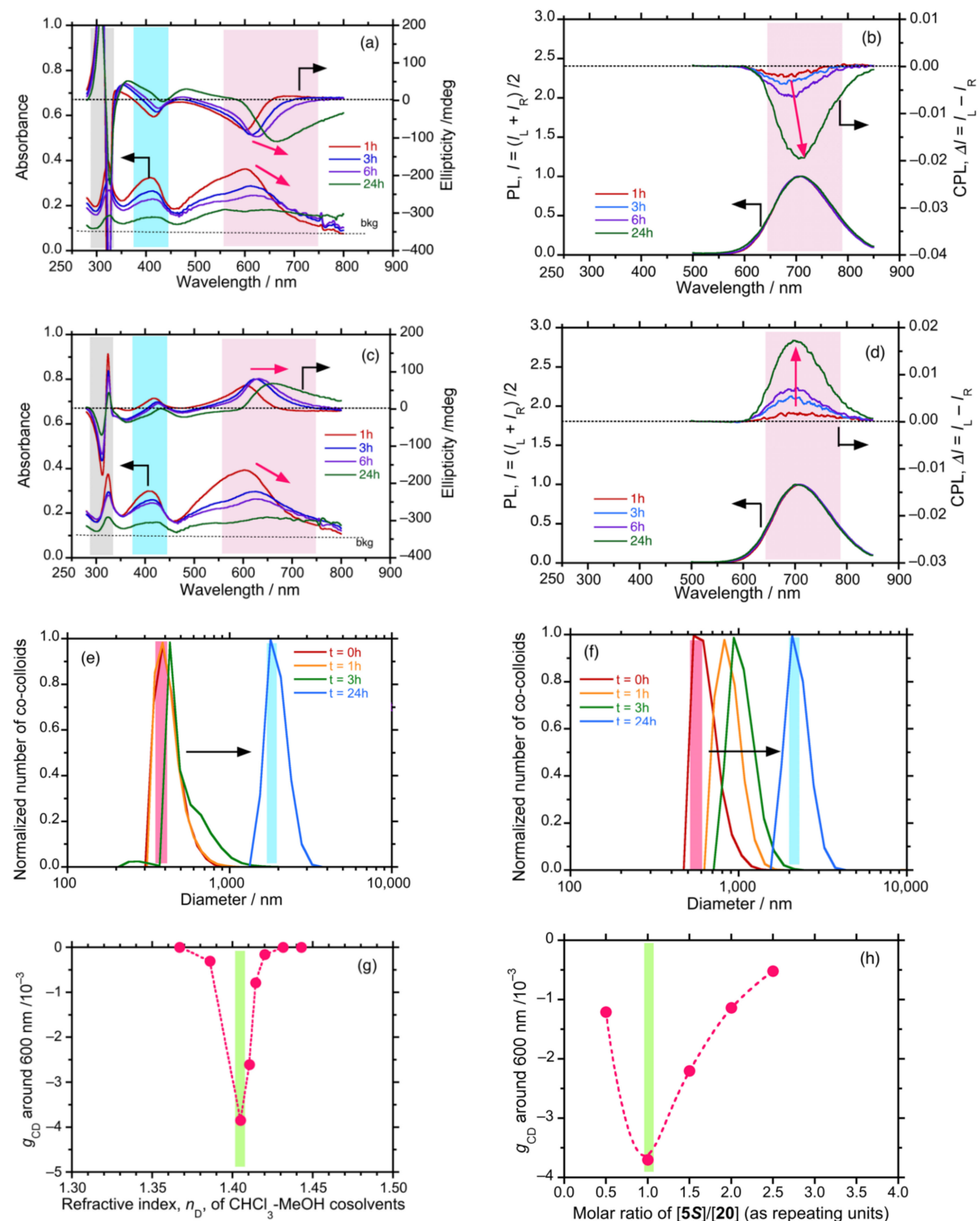

Figure 17. Alteration in the (a) CIE-CD and UV-visible-NIR spectra and (b) CIE-CPL and PL spectra excited at $400 \mathrm{~nm}$ for the $\mathbf{5 S}-\mathbf{2 0}$ co-colloids (1-to- 1 molar ratio). Alteration in the (c) CIE-CD and UVvisible-NIR spectra and (d) CIE-CPL and PL spectra excited at $400 \mathrm{~nm}$ for the $\mathbf{5 R - 2 0}$ co-colloids (1-to-1 molar ratio). These co-colloids were produced in $\mathrm{CHCl}_{3}-\mathrm{MeOH}(2 / 1(\mathrm{v} / \mathrm{v}))$. The hydrodynamic sizes of (e) 5S-20 and (b) 5R-20 co-colloids with the propagation time; (g) the $g_{\mathrm{CD}}$ value around $600 \mathrm{~nm}$ of the 20-5S co-colloid (1:1) as a function of $n_{\mathrm{D}}$ in the $\mathrm{CHCl}_{3}-\mathrm{MeOH}$ cosolvent; (h) the $g_{\mathrm{CD}}$ value of the $\mathbf{2 0}-\mathbf{5 S}$ co-colloid as a function of the $5 S$-to- 20 ratio generated in the $\mathrm{CHCl}_{3}-\mathrm{MeOH}(2: 1)$ cosolvent. Modified from an original article [210].

4.7. Unveiling Anti-Kasha's Rule from CIE-CPL, CIE-CPLE, and CIE-CD in Co-Colloids of Achiral $\pi$-Conjugated Polymer and Helical Polysilanes

In this section, we deal with the origin of bisignate CIE-CPL associated with bisignate CIE-CD spectra characteristic of the co-colloids comprising $\mathbf{1 1}$ with $\mathbf{5} \boldsymbol{R}(1: 1 \mathrm{~mol}$ ratio) and 11 with $5 S$ (1:1 mol ratio) by connecting to the corresponding CIE-CPLE spectra based on a hypothesis of anti-Kasha's rule to explain the origin of dual CPL emission behaviors. Note that $5 R$ and $5 S$ can work as $P$-screw and $M$-screw helical scaffoldings in the range of 280$325 \mathrm{~nm}$ to achiral 11 in the range of $350-650 \mathrm{~nm}$. Prior to a series of chiroptical experiments, the $n_{\mathrm{D}}$ value of the $\mathrm{CHCl}_{3}-\mathrm{MeOH}$ cosolvents is optimized (Figure $18 \mathrm{e}$ ); $n_{\mathrm{D}}=1.410$ of the 
$\mathrm{CHCl}_{3} / \mathrm{MeOH}(2.1 / 0.9(\mathrm{v} / \mathrm{v}))$ cosolvent for $\mathbf{1 1} / \mathbf{5 R}=1: 1$ in mole ratio and $n_{\mathrm{D}}=1.415$ of the $\mathrm{CHCl}_{3}-\mathrm{MeOH}$ cosolvent $(2.2 / 0.8(\mathrm{v} / \mathrm{v}))$ cosolvent for 11/5S = 1:1 in mole ratio, respectively.
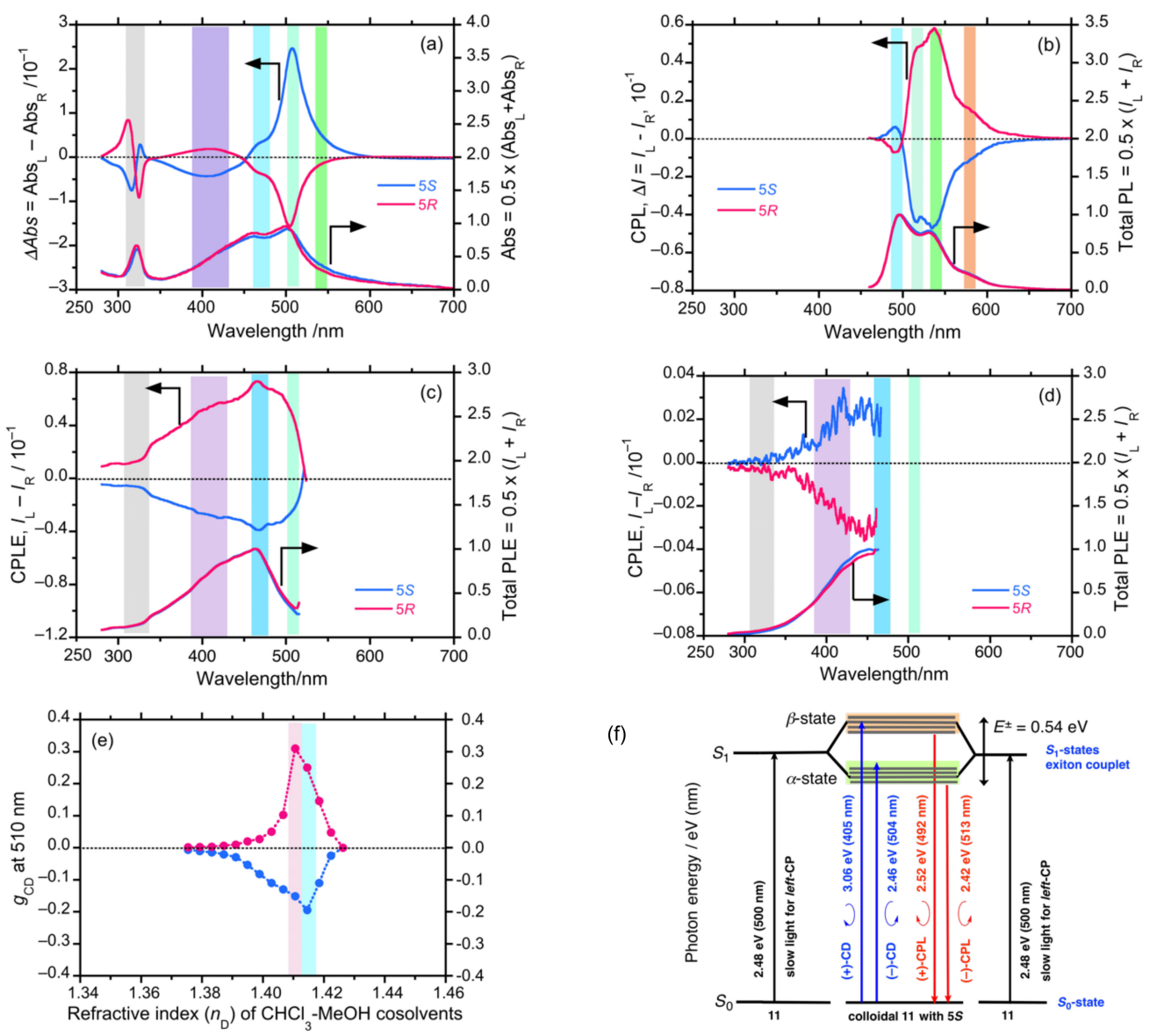

(f)

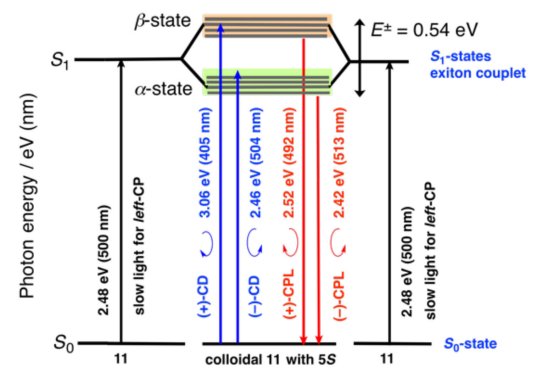

Figure 18. (a) The CIE-CD and UV-visible spectra and (b) the CIE-CPL and PL spectra excited at $420 \mathrm{~nm}$ of the co-colloids of $\mathbf{1 1}$ with $5 S$ and $5 R$ in a 1-to- 1 molar ratio; (c) The CIE-CPLE and PLE spectra of the co-colloids of $\mathbf{1 1}$ with $5 S$ and $5 R$ monitored at (d) $575 \mathrm{~nm}$ and (e) $490 \mathrm{~nm}(5 R)$ in the $\mathrm{CHCl}_{3}-\mathrm{MeOH}$ cosolvent $(2.2 / 0.8(\mathrm{v} / \mathrm{v}))$ and (d) $575 \mathrm{~nm}$ and (e) $495 \mathrm{~nm}$ (5S) in the $\mathrm{CHCl}_{3}-\mathrm{MeOH}$ $(2.1 / 0.9(\mathrm{v} / \mathrm{v})) ;(\mathbf{e})$ the $g_{\mathrm{CD}}$ value at $500 \mathrm{~nm}$ of the co-colloidal 11-5S (1:1) as a function of $n_{\mathrm{D}}$ in the $\mathrm{CHCl}_{3}-\mathrm{MeOH}$ cosolvent; (f) A hypothesis of anti-Kasha's rule and Jablonski diagram to explain bisignate CIE-CD, CIE-CPL, and CIE-CPLE spectra of the colloidal 11 endowed with 5S. Modified from an original article [209].

Figure 18a,b depicts the CIE-CD/UV-visible and CIE-CPL/UV-PL spectra excited at $420 \mathrm{~nm}$ of the co-colloids consisting of 11/5R (1:1) and 11/5S (1:1), respectively. In Figure $18 \mathrm{a}$, the weaker bisignate CIE-CD/UV spectra around $300 \mathrm{~nm}$ are attributed to Si $\sigma-\mathrm{Si}^{*}$ transitions of $\mathbf{5 R}$ and $\mathbf{5 S}$. In Figure $18 \mathrm{a}$, it is obvious that the intense bisignate CIE-CD/UVvisible spectra at 400 and $500 \mathrm{~nm}$ are attributed to $\pi-\pi^{*}$ transitions of $\mathbf{1 1}$ led by $5 R$ and 11 led by $5 S$, respectively. However, from Figure $18 \mathrm{~b}$, the intense bisignate, dual CIE-CPL bands associated with PL band located at $490 \mathrm{~nm}$ and $510-580 \mathrm{~nm}$ are assumed to originate from $\pi-\pi^{*}$ transitions of the colloidal 11 with $5 R$ and $5 S$. One question is whether the bisignate CPL, not obvious from PL, arises from $\pi-\pi^{*}$ transitions at $400 \mathrm{~nm}$ and $500 \mathrm{~nm}$ from the colloidal 11. To answer this query, we obtained the first CIE-CPLE/PLE spectra monitored at $575 \mathrm{~nm}$ and at 480-480 nm (Figure 15c,d). The (+)-sign CIE-CPLE/PLE spectra of the 11-5R monitored at $575 \mathrm{~nm}$ are nearly identical to (+)-sign CD spectra of the $\pi-\pi^{*}$ transitions of the $\mathbf{1 1}-\mathbf{5 R}$ in the range of $300-500 \mathrm{~nm}$ and vice versa (Figure 15c). Conversely, the (-)-sign CIE-CPLE/PLE spectra of the 11-5R monitored at 480-490 nm are 
nearly identical to (-)-sign CD spectra of the $\pi-\pi^{*}$ transitions of the $11-5 R$ in the range of $300-450 \mathrm{~nm}$ and vice versa (Figure 15d).

These spectral results allow us to propose the anti-Kasha's rule, similar to Section 4.5.3. Two pathways of spontaneous relaxation processes from the $\beta$-state in higher energy ( $\sim 400$ $\mathrm{nm})$ and the $\alpha$-state with lower energy $(\sim 500 \mathrm{~nm})$ are possible. The $r$-CP light in the range of 350 and $500 \mathrm{~nm}$ photoexcited the 11-5S (and 11-5R), followed by relaxation to the $\beta$ - and $\alpha$-states that emit $r$-CPL and $l$-CPL, respectively. On the other hand, the $r$-CP light at $500 \mathrm{~nm}$ photoexcited the 11-5S (and 11-5R), followed by relaxation to the $\alpha$-states that emit $l$-CPL only. The photoexcited $\beta$-state with $r$-CP light has two relaxation pathways; from $\beta$-state with $r$-CP light and from $\alpha$-state with $l$-CP light.

The $r$ - and $l$-CP light excitation processes, followed by spontaneous radiation processes, may not obey the conventional Kasha's rule, rather obey anti-Kasha's rule because dual $\mathrm{CP}$ luminescence characteristics are obvious. In recent years, anti-Kasha's rule became popular and is now a hot topic in the realm of photophysics and photochemistry when dual and multiple photoluminescence characteristics are observed [238-241]. Monitorwavelength dependent CPLE spectroscopy associated with the corresponding CPL and CD spectroscopy is helpful to investigate the anti-Kasha's rule in solutions and in the solid film, and as solid powders.

\section{Perspectives-Colloids Connecting to Light, Helix, Coacervate, Panspermia, Microoptics, Chiroptics, Radioisotopes, Nuclear Physics, Biology, Homochirality Question, and Cosmology}

With assumptions based on the coacervate and Panspermia hypotheses, and WGM in $\mu \mathrm{m}$-size colloidal polymers adaptable to external chemical and physical stimuli in a tuned optofluidic media, one remaining issue should be to address the effects of chiral physical sources and particles existing in the interstellar universe and on Earth, possibly causing the biomolecular handedness on Earth.

First, the author wishes to mention personal experience in my graduate school four decades ago. In 1977, Kunitake and Okahata found that didodecyldimethylammonium bromide in water, the simplest amphiphilic synthetic molecule (Figure 19, top left), spontaneously generates multilayer spherical colloids of $\sim 100 \mathrm{~nm}$ in diameter from observation of TEM [242]. Under supervision of Kunitake and his three lab staffers (Takarabe, Okahata, and Shinkai), the author designed four new achiral, amphiphilic acrylic monomers (Figure 19, top right), polymerizable under influence of the $\beta^{-}$-decay process from ${ }^{60} \mathrm{Co} \rightarrow{ }^{60} \mathrm{Ni}$ nuclear fission reaction [243].

Among the four amphiphiles, $\mathrm{AcC}_{10} \mathrm{C}_{12} \mathrm{~N}^{+} 2 \mathrm{C}_{1} \mathrm{Br}$ in water, showing two phase-transition temperatures $\left(T_{\mathrm{C}} \mathrm{s}\right)$ at $-16{ }^{\circ} \mathrm{C}$ and $-9{ }^{\circ} \mathrm{C}$, formed structureless colloids with no obvious multilayers, ranging from $\sim 10 \mathrm{~nm}$ to $\sim 100 \mathrm{~nm}$ in diameter (Figure 19a). Followed by polymerization of the colloids embedded to ice in the range of $-30^{\circ} \mathrm{C}$ and $-50^{\circ} \mathrm{C}$ with help from the $\beta^{-}$-decay nuclear reaction, the author serendipitously observed that the spiral fibers of amphiphilic polyacrylate ( $10 \mathrm{~nm}$ in diameter) are formed in many places. Possibly, handedness of spiral fibers was left-handed (Figure 19b-d). Polymerization was confirmed by significant shifts in IR frequency in $v_{(\mathrm{C}=\mathrm{O})}$ from $1715 \mathrm{~cm}^{-1}$ (monomer) and $1725-1730 \mathrm{~cm}^{-1}$ (polymer). Likewise, $\mathrm{AcC}_{10} \mathrm{C}_{18} \mathrm{~N}^{+} 2 \mathrm{C}_{1} \mathrm{Br}$ in water exhibited two phase-transition temperatures $\left(T_{\mathrm{c}} \mathrm{s}\right)$ at $28^{\circ} \mathrm{C}$ and $41{ }^{\circ} \mathrm{C}$. Colloidal $\mathrm{AcC}_{10} \mathrm{C}_{18} \mathrm{~N}^{+} 2 \mathrm{C}_{1} \mathrm{Br}$ polymerized at $45^{\circ} \mathrm{C}$ influenced by the $\beta^{-}$-decay reaction formed clearly loop-like structures (ca. $40-60 \mathrm{~nm}$ ) made of spiral fiber (Figure 19e,f). The handed spiral loops from the polymerizable colloids by dosing handed electron, $\gamma$-rays, and anti-neutrino have very reminiscent circular DNAs and proteins/polypeptides influenced by high-energy cosmic rays under extraterrestrial and terrestrial conditions of primordial eons. 

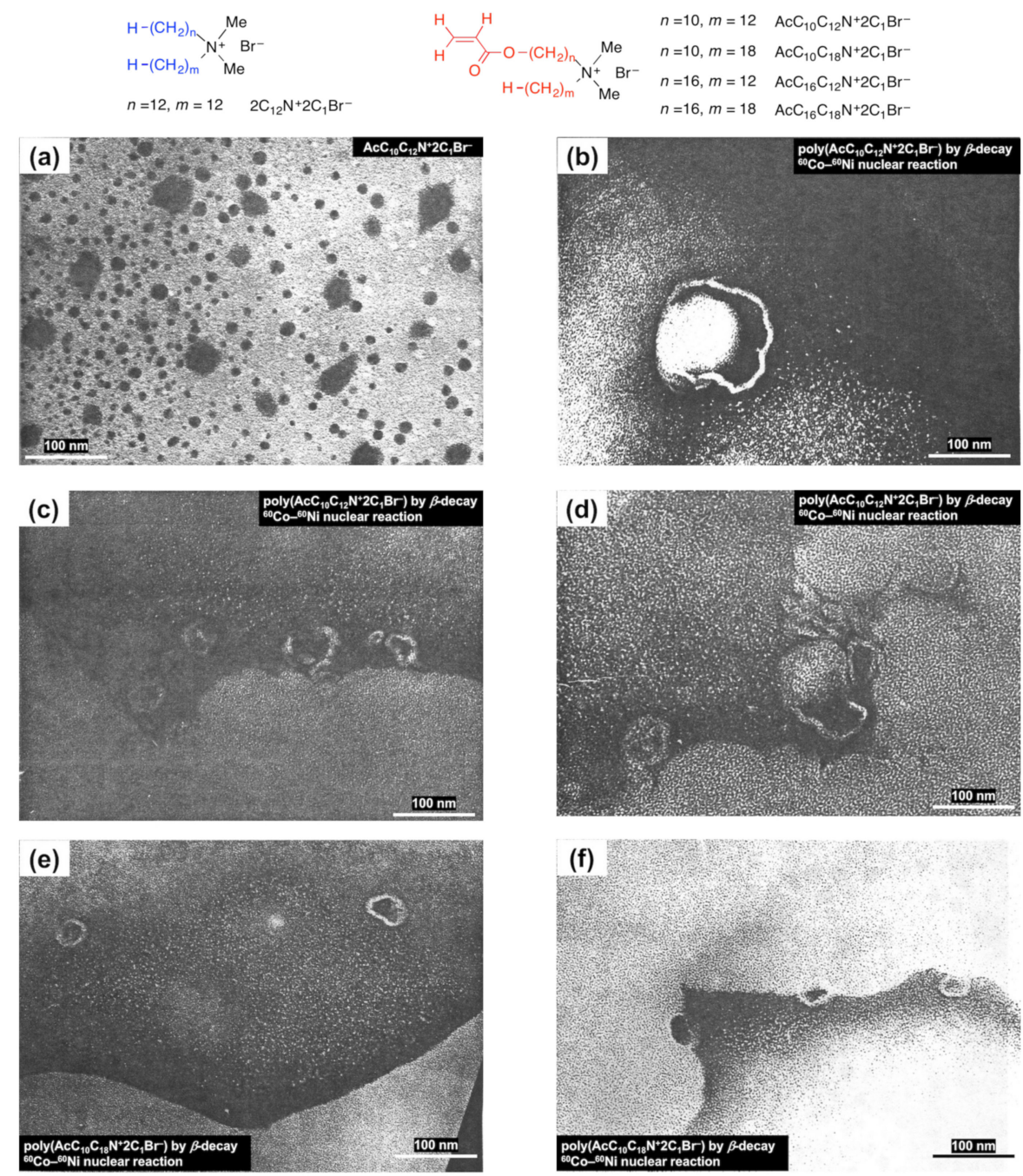

Figure 19. (Top, left) Chemical structures of the first amphiphilic didodecyldimethylammonium bromide to spontaneously form spherical shape colloids ( 100 nm) with multilayer membrane [242]. (Top, right) Four amphiphilic acrylic monomers synthesized by the author (1978) (unpublished as and English paper, disclosed only as his Master thesis [243]. A TEM image (negative statue) of (a) colloidal $\mathrm{AcC}_{10} \mathrm{C}_{12} \mathrm{~N}^{+} 2 \mathrm{C}_{1 \mathrm{Br}^{-}}(20 \mathrm{mM})$ (negative statue) dispersed in pure water; (b-d) TEM images (positive statue) for the colloidal particles polymerized in the range of $-30{ }^{\circ} \mathrm{C}$ and $-50{ }^{\circ} \mathrm{C}$ endowed with $\beta$-decay of ${ }^{60} \mathrm{Co} \rightarrow{ }^{60} \mathrm{Ni}$ nuclear fission reaction; dose rate: $20 \mathrm{R}\left(5.2 \times 10^{-3} \mathrm{C} \mathrm{kg}^{-1}\right)$ per s for $50 \mathrm{~h}$, corresponding to $3.6 \times 10^{6} \mathrm{R}\left(9.3 \times 10^{2} \mathrm{C} \mathrm{kg}^{-1}\right)$; (e,f) TEM images (positive statue) for closed loop shape structures from colloidal $\mathrm{AcC}_{10} \mathrm{C}_{18} \mathrm{~N}^{+} 2 \mathrm{C}_{1 \mathrm{Br}}^{-}$polymerized at $45^{\circ} \mathrm{C}$ led by $\beta$-decay of ${ }^{60} \mathrm{Co} \rightarrow{ }^{60} \mathrm{Ni}$ nuclear reaction; dose rate: $20 \mathrm{R}\left(5.2 \times 10^{-3} \mathrm{C} \mathrm{kg}^{-1}\right)$ per s for $3.5 \mathrm{~h}$, corresponding to $2.6 \times 10^{5} \mathrm{R}\left(0.6 \times 10^{2} \mathrm{C} \mathrm{kg}^{-1}\right)$. These TEM images suggested spiral fibers dispatching from the colloidal polymers [243]. A Hitachi (Ibaraki, Japan) model H-500 TEM instrument was employed with an accelerating voltage of $100 \mathrm{kV}$ at lab of Motoo Takayanagi (Kyushu University). All figures (a-f) were scanned from the Master thesis [243].

The author conjectured at that time that handed spiral from the colloids in the ultracold and hot conditions is a model of the cometary coacervates hypothesis in comets [123]. Three chiral physical sources are possible to connect to the spiral rings because the $\beta^{-}$-decay process from ${ }^{60} \mathrm{Co}$ radiates $l$-hand spinning $\beta$-electron, $r$-hand $\gamma$-ray (high-energy circularly polarized light), and $l$-hand anti-neutrino simultaneously from the source [69-76].

Amphiphilic colloidal particles in water may feel the forces led by these handed radioactive physical sources because atmospheric thundering and radioactive materials in Earth's crust and mantle might be ubiquitous in primordial eons, as well as the present 
days. Similarly, microdroplets in aqueous solution and aqueous aerosols in the atmosphere may be susceptible to these handed physical sources, leading to accelerated chemical reactions led by optofluidic WGM scenarios.

In recent years, several review papers on terrestrial and extraterrestrial life, chemical evolution, the origin of life, and the origin of chirality on Earth have been disclosed [11-21,244-247]. These papers comprehensively cover curious topics in the realms of astrochemistry, astrobiology, geoscience, space life science, synthetic biology, origin of life, central dogma, natural science, and materials science. The reviews should provide new insights in the realms of helical polymer chemistry [248], supramolecular chemistry [249], flow chemistry with light [250], natural product chemistry [251], and chiroptical polymer chemistry [252]. However, possible connections of these topics with nonequilibrium open-flow colloidal systems seem to be rare. An importance of (chir)optical characteristics of surrounding media, degree of smooth interfaces, micro-optics and optofluidics, photon confinement, resonance condition, WGM, and nature of light-matter interactions in the presence and absence of static electric field and static magnetic field are not well recognized. In my view, when one can setup open-flow, cell-wall-free $\mu \mathrm{m}$-size colloidal polymers with a smooth surface dispersed in aqueous conditions with tuned RIs, any mirror-symmetrical physical forces and non-mirror-symmetrical inherently chiral particles, such as solar neutrinos and anti-neutrinos, are possible to induce and boost the left-right imbalance disregard of terrestrial and extraterrestrial conditions, even at cryogenic temperatures. The concept of the open-flow, non-equilibrium optofluidic colloidal and co-colloidal systems shed light at opening new science and engineering opportunities in chirogenesis and photochirogenesis, in addition to conventional stereochemistry, photochemistry, and photophysics in homogeneous solutions. The ideas are applicable to chirogenesis and photochirogenesis in $\mu \mathrm{m}$-size water-based aerosols and microdroplets to address plausible scenarios for the homochirality questions on Earth.

\section{Conclusions}

Colloids and co-colloids with higher RI dispersed in optofluidic mediums with lower $\mathrm{RI}$ are capable of chiroptical resonators susceptible of external chiral chemical and $\mathrm{CP}$ light sources. Concerning $\mu \mathrm{m}$-size colloids, made of $\pi$ - $/ \sigma$-conjugated polymers in optofluidic medium, with a tuned RI, several resonance effects cause efficient chirogenesis and photochirogenesis, revealed by gigantic, enhanced CD, ORD, CPL, and CPLE spectral datasets. The chirogenesis is susceptible to the nature of optofluidic medium, including chiral chemical substances and a ratio of $\pi$ - and $\sigma$-conjugated polymers. Moreover, a fully controlled absolute photochirogenesis upon excitation of left- and right-CP light sources at several wavelengths is a possible disregard of photochromic and non-photochromic polymers at specific refractive indices of optofluidic media. The wavelength-dependent $\mathrm{CP}$ light source can fully drive all chiroptical generation, inversion, erase, switching, and short-/long-lived memories. New knowledge and deep understanding of CPL, CPLE, CD, and ORD spectroscopy for colloids and co-colloids can shed light on rationally designing elaborate CPL functioned $\pi-/ \sigma$-conjugated polymers and metal-coordination polymers in the future. A tandem analysis of CPLE, CPL, and CD spectroscopy helps to discuss Kasha's rule and anti-Kasha's rule of radiation processes from the photoexcited optically active molecules, polymers, colloids, solids, crystals, and thin films. Our comprehensive studies, using artificial polymeric luminophores and chromophores, should provide new insight of the open-flow, non-equilibrium functional colloids, and co-colloids in the photoexcited and ground states in an RI-tuned optofluidic medium. Based on the results shown above, and my personal experiences, any chiral physical forces and particles could induce and boost the left-right imbalance, which is one possible answer to the homochirality question on Earth's disregard of terrestrial and extraterrestrial origin chirality. In particular, when open-flow, $\mu \mathrm{m}$-size colloidal polymers with smooth surfaces, adaptable to external physical and chemical biases are surrounded by an optofluidic medium, such as an aqueous solution, the capabilities of chiral physical forces and chiral chemical sources are possible, 
to enhance by huge numbers of recycling times $(N)$ in the optofluidic colloidal polymers, in line with the scheme of WGM. This means that a subtle left-right imbalance interior of the colloid, e.g., $10^{-6 \%} e e$, is boostable by $10^{-6} \times \mathrm{N}$. This scenario is in contrast to a conventional stereochemistry, photochemistry, and photophysics in a homogeneous solution system, in which the light-matter interaction occurs in one time $(N=1)$ only by assuming the Beer-Lambert law in homogeneous solutions. When colloid-induced emission systems chiroptically fulfil the resonance condition, one can anticipate the significant enhancements in CPL and CD signals.

Funding: The author is grateful for the financial support from a Grant-in-Aid for Scientific Research (16655046, 21655041, 22350052, 23651092, 26620155, and 16H04155).

Institutional Review Board Statement: Not applicable.

Informed Consent Statement: Not applicable.

Data Availability Statement: Excepting Figures 16 and 19, no new data were created or analyzed in this study. Data sharing is not applicable to this article. Figures 16 and 19 are available on request from the corresponding author.

Acknowledgments: First of all, the author owes a debt of gratitude to Rui Tamura (Kyoto University) for the opportunity to contribute our CD/ORD/CPL/CPLE spectroscopic studies of the colloidal $\sigma-/ \pi$-conjugated polymers at NTT and NAIST with my colleagues and students, over 20 years, to address the curious homochirality questions on Earth. The author expresses special thanks to his coworkers; Hiroshi Nakashima, Seiji Toyoda, Kyozaburo Takeda, Hiromi Takigawa-Tamoto, Masao Motonaga, Yoshihiro Kimura, Fumiko Ichiyanagi, Yoko Nakano, Yang Liu, Takashi Mori, Yoshifumi Kawagoe, Kana Yoshida, Ayako Nakao, Nozomu Suzuki, Keisuke Yoshida, Yuri Donguri, Yuka Kato, Makoto Taguchi, Sang Thi Duong, Mohamed Mehawed Abdellatif, Ai Yokokura, Nor Azura Abdul Rahim, Abd Jalil Jalilah, Laibing Wang, Shosei Yoshimoto, Sibo Guo, Yota Katsurada, Seiko Amazumi, Hailing Chen, Nanami Ogata, Kazuki Yamasaki, Asuka Okubo, Shun Okazaki, Hiroki Kamite, Puhup Puneet, Anubhav Saxena, Takumi Yamada, Nobuyuki Hara, Yuki Mimura, Wei Zhang, Yonggang Yang, Julian R. Koe, Ken Terao, the late Akio Teramoto, Yoshitane Imai, Kotohiro Nomura. The present paper, as my life work, was initiated by irreplaceable experiences, knowledge, serendipitous findings, and unanswered questions at Kyushu University (1976-1978) under supervisions of four scholars, Toyoki Kunitake, Kunihide Takarabe, Yoshio Okahata, and Seiji Shinkai. Finally, the author expresses special thanks to the three anonymous reviewers for their stimulating and critical comments.

Conflicts of Interest: The author declares no conflict of interest.

\section{References}

1. Schrodinger, E. What Is Life?: With Mind and Matter and Autobiographical Sketches; Cambridge University Press: Cambridge, UK, 1944.

2. Mason, S.F. Molecular Optical Activity and the Chiral Discriminations; Cambridge University Press: Cambridge, UK, 1982.

3. Mason, S.F.; Tranter, G.E. The Electroweak Origin of Biomolecular Handedness. Proc. R. Soc. Lond. A 1985, 397, $45-65$.

4. Bonner, W.A. Terrestrial and Extraterrestrial Sources of Molecular Homochirality. Orig. Life Evol. Biosph. 1992, 21, 407-420. [CrossRef]

5. Bailey, J.; Chrysostomou, A.; Hough, J.H.; Gledhill, T.M.; McCall, A.; Clark, S.; Ménard, F.; Tamura, M. Circular Polarization in Star-Formation Regions: Implications for Biomolecular Homochirality. Science 1998, 281, 672-674. [CrossRef] [PubMed]

6. Avalos, M.; Babiano, R.; Cintas, P.; Jiménez, J.L.; Palacios, J.C. From Parity to Chirality: Chemical Implications Revisited. Tetrahedron Asymmetry 2000, 11, 2845-2874. [CrossRef]

7. Nishino, H.; Kosaka, A.; Hembury, G.A.; Aoki, F.; Miyauchi, K.; Shitomi, H.; Onuki, H.; Inoue, Y. Absolute Asymmetric Photoreactions of Aliphatic Amino Acids by Circularly Polarized Synchrotron Radiation: Critically pH-Dependent Photobehavior. J. Am. Chem. Soc. 2002, 124, 11618-11627. [CrossRef] [PubMed]

8. Kawasaki, T.; Sato, M.; Ishiguro, S.; Saito, T.; Morishita, Y.; Sato, I.; Nishino, H.; Inoue, Y.; Soai, K. Enantioselective Synthesis of Near Enantiopure Compound by Asymmetric Autocatalysis Triggered by Asymmetric Photolysis with Circularly Polarized Light. J. Am. Chem. Soc. 2005, 127, 3274-3275. [CrossRef]

9. Guijarro, A.; Yus, M. Origin of Chirality in the Molecules of Life: A Revision from Awareness to the Current Theories and Perspectives of this Unsolved Problem; RSC Publishing: Cambridge, UK, 2008; ISBN 978-0-85404-156-5. 
10. Plasson, R.; Kondepudi, D.K.; Bersini, H.; Commeyras, A.; Asakura, K. Emergence of Homochirality in Far-From-Equilibrium Systems: Mechanisms and Role in Prebiotic Chemistry. Chirality 2007, 19, 589-600. [CrossRef]

11. Macdermott, A.J. Chiroptical Signatures of Life and Fundamental Physics. Chirality 2012, 24, 764-769. [CrossRef]

12. Liu, M.; Zhang, L.; Wang, T. Supramolecular Chirality in Self-Assembled Systems. Chem. Rev. 2015, 115, 7304-7397. [CrossRef]

13. Davankov, V.A. Biological Homochirality on the Earth, or in the Universe? A Selective Review. Symmetry 2018, 10, 749. [CrossRef]

14. Famiano, M.; Boyd, R.; Kajino, T.; Onaka, T.; Mo, Y. Astrophysical Sites that Can Produce Enantiomeric Amino Acids. Symmetry 2019, 11, 23. [CrossRef]

15. Tamura, M.; Kandori, R.; Kusakabe, N.; Nakajima, Y.; Hashimoto, J.; Nagashima, C.; Nagata, T.; Nagayama, T.; Kimura, H.; Yamamoto, T.; et al. Near-infrared polarization images of the Orion nebula. Astrophys. J. Lett. 2006, 649, L29-L32. [CrossRef]

16. Takahashi, J.-I.; Kobayashi, K. Origin of Terrestrial Bioorganic Homochirality and Symmetry Breaking in the Universe. Symmetry 2019, 11, 919. [CrossRef]

17. Ribó, J.M.; Hochberg, D. Chemical Basis of Biological Homochirality during the Abiotic Evolution Stages on Earth. Symmetry 2019, 11, 814. [CrossRef]

18. Suzuki, N.; Itabashi, Y. Possible Roles of Amphiphilic Molecules in the Origin of Biological Homochirality. Symmetry 2019, 11, 966. [CrossRef]

19. Sang, Y.; Liu, M. Symmetry Breaking in Self-Assembled Nanoassemblies. Symmetry 2019, 11, 950. [CrossRef]

20. Soai, K.; Kawasaki, T.; Matsumoto, A. Role of Asymmetric Autocatalysis in the Elucidation of Origins of Homochirality of Organic Compounds. Symmetry 2019, 11, 694. [CrossRef]

21. Fujiki, M. Mirror Symmetry Breaking in Helical Polysilanes: Preference Between Left and Right of Chemical and Physical Origin. Symmetry 2010, 2, 1625-1652. [CrossRef]

22. Akabori, S.; Okawa, K.; Sato, M. Introduction of Side Chains into Polyglycine Dispersed on Solid Surface. I. Bull. Chem. Soc. Jpn. 1956, 29, 608-611. [CrossRef]

23. Rohlfing, D.L.; Oparin, A.I. (Eds.) Molecular Evolution: Prebiological and Biological; Plenum Press: New York, NY, USA, 1972.

24. Sparks, W.B.; Hough, J.H.; Kolokolova, L.; Germer, T.A.; Chen, F.; DasSarma, S.; DasSarma, P.; Robb, F.T.; Manset, N.; Reid, I.N.; et al. Circular Polarization in Scattered Light as a Possible Biomarker. J. Quant. Spectrosc. Radiat. Transf. 2009, 110, 1771-1779. [CrossRef]

25. Pizzarello, S. Molecular Asymmetry in Prebiotic Chemistry: An Account from Meteorites. Life 2016, 6, 18. [CrossRef] [PubMed]

26. Morrison, S.M.; Runyon, S.E.; Hazen, R.M. The Paleomineralogy of the Hadean Eon Revisited. Life 2018, 8, 64. [CrossRef] [PubMed]

27. Kirschvink, J.L.; Gaidos, E.J.; Bertani, L.E.; Beukes, N.J.; Gutzmer, J.; Maepa, L.N.; Steinberger, R.E. Paleoproterozoic Snowball Earth: Extreme Climatic and Geochemical Global Change and Its Biological Consequences. Proc. Natl. Sci. Acad. USA 2000, 97, 1400-1405. [CrossRef] [PubMed]

28. Rooney, A.D.; Macdonald, F.A.; Strauss, J.V.; Dudás, F.O.; Hallmann, C.; Selby, D. Re-Os Geochronology and Coupled Os-Sr Isotope Constraints on the Sturtian Snowball Earth. Proc. Natl. Sci. Acad. USA 2014, 111, 51-56. [CrossRef]

29. Ueno, Y.; Yamada, K.; Yoshida, N.; Maruyama, S.; Isozaki, Y. Evidence from Fluid Inclusions for Microbial Methanogenesis in the Early Archaean Era. Nature 2006, 440, 516-519. [CrossRef]

30. Nojzsis, S.J.; Arrhenius, G.; McKeegan, K.D.; Harrison, T.M.; Nutman, A.P.; Friend, C.R. Evidence for Life on Earth before 3800 Million Years Ago. Nature 1996, 384, 55-59.

31. Hoffman, P.F.; Schrag, D.P. The Snowball Earth Hypothesis: Testing the Limits of Global Change. Terra Nova 2002, 14, 129-155. [CrossRef]

32. Okamoto, Y.; Kakegawa, T.; Ishida, A.; Nagase, T.; Rosing, M.T. Evidence for Biogenic Graphite in Early Archaean Isua Metasedimentary Rocks. Nat. Geosci. 2014, 7, 25-28.

33. Tarduno, J.A.; Cottrell, R.D.; Davis, W.J.; Nimmo, F.; Bono, R.K. A Hadean to Paleoarchean Geodynamo Recorded by Single Zircon Crystals. Science 2015, 349, 521-524. [CrossRef]

34. Tang, F.; Taylor, R.J.; Einsle, J.F.; Borlina, C.S.; Fu, R.R.; Weiss, B.P.; Williams, H.M.; Williams, W.; Nagy, L.; Midgley, P.A.; et al. Secondary Magnetite in Ancient Zircon Precludes Analysis of a Hadean Geodynamo. Proc. Natl Acad. Sci. USA 2019, 116, 407-412. [CrossRef]

35. Witze, A. Greenland Rocks Suggest Earth's Magnetic Field Is Older Than We Thought. Nature 2019, 576, 347. [CrossRef] [PubMed]

36. Zawaski, M.J.; Kelly, N.M.; Orlandini, O.F.; Nichols, C.I.O.; Allwood, A.C.; Mojzsis, S.J. Reappraisal of Purported ca. 3.7 Ga Stromatolites from the Isua Supracrustal Belt (West Greenland) from Detailed Chemical and Structural Analysis. Earth Planet. Sci. Lett. 2020, 545, 116409. [CrossRef]

37. Epstein, S.; Krishnamurthy, R.V.; Cronin, J.R.; Pizzarello, S.; Yuen, G.U. Unusual Stable Isotope Ratios in Amino Acid and Carboxylic Acid Extracts from the Murchison Meteorite. Nature 1987, 326, 477-479. [CrossRef] [PubMed]

38. Engel, M.H.; Macko, S.A. Isotopic Evidence for Extraterrestrial Non-racemic Amino Acids in the Murchison Meteorite. Nature 1987, 389, 265-268. [CrossRef] [PubMed]

39. Frieden, B.R.; Plastino, A.; Plastino, A.R.; Soffer, B.H. Schrodinger Link Between Nonequilibrium Thermodynamics and Fisher Information. Phys. Rev. E 2002, 66, 046128. [CrossRef]

40. Ornes, S. Core Concept: How Nonequilibrium Thermodynamics Speaks to The Mystery of Life. Proc. Natl. Acad. Sci. USA 2017, 114, 423-424. [CrossRef] 
41. Ribó, J.M.; Hochberg, D.; Crusats, J.; El-Hachemi, Z.; Moyano, A. Spontaneous Mirror Symmetry Breaking and Origin of Biological Homochirality. J. R. Soc. Interface 2017, 14, 20170699. [CrossRef]

42. Goldanskiǐ, V.I.; Kuz'min, V.V. Spontaneous Breaking of Mirror Symmetry in Nature and the Origin of Life. Sov. Phys. Usp. 1989, 32, 1-29. [CrossRef]

43. Avetisov, V.A.; Goldanskii, V.I.; Kuz'min, V.V. Handedness, Origin of Life and Evolution. Phys. Today 1991, $44,33-41$.

44. Avetisov, V.; Goldanskii, V. Mirror Symmetry Breaking at the Molecular Level. Proc. Natl. Acad. Sci. USA 1996, 93, 11435-11442. [CrossRef]

45. Frank, F.C. On Spontaneous Asymmetric Synthesis. Biochim. Biophys. Acta 1953, 11, 459-463. [CrossRef]

46. Kuhn, W.; Brown, E. Photochemische Erzeugung Optisch Aktiver Stoffe. Naturwissenschaften 1929, 17, 227-228. [CrossRef]

47. Rau, H. Asymmetric Photochemistry in Solution. Chem. Rev. 1983, 83, 535-547. [CrossRef]

48. Inoue, Y. Asymmetric Photochemical Reactions in Solution. Chem. Rev. 1992, 92, 741-770. [CrossRef]

49. Rau, H. Direct Asymmetric Photochemistry with Circularly Polarized light. In Chiral Photochemistry: Molecular and Supramolecular Photochemistry; Inoue, Y., Ramamurthy, V., Eds.; Marcel Dekker: New York, NY, USA, 2004; Chapter 1; pp. 1-44.

50. Meierhenrich, U.J.; Nahon, L.; Alcaraz, C.; Bredehöft, J.H.; Hoffmann, S.V.; Barbier, B.; Brack, A. Asymmetric Vacuum UV photolysis of the Amino Acid Leucine in the Solid State. Angew. Chem. Int. Ed. 2005, 44, 5630-5634. [CrossRef]

51. INOUE Photo-Chirogenesis. Available online: https://www.jst.go.jp/erato/en/research_area/completed/ihh_P.html (accessed on 22 January 2021).

52. Bernstein, W.J.; Calvin, M.; Buchardt, O. Absolute Asymmetric Synthesis. I. Mechanism of the Photochemical Synthesis of Nonracemic Helicenes with Circularly Polarized Light. Wavelength Dependence of the Optical Yield of Octahelicene. J. Am. Chem. Soc. 1972, 94, 494-498. [CrossRef]

53. Meinert, C.; Hoffmann, S.V.; Cassam-Chenaï, P.; Evans, A.C.; Giri, C.; Nahon, L.; Meierhenrich, U.J. Photonenergy-Controlled Symmetry Breaking with Circularly Polarized Light. Angew. Chem. Int. Ed. 2014, 53, 210-214. [CrossRef]

54. Fujiki, M.; Yoshida, K.; Suzuki, N.; Zhang, J.; Zhang, W.; Zhu, X. Mirror Symmetry Breaking and Restoration within $\mu \mathrm{m}-$ Sized Polymer Particles in Optofluidic Media by Pumping Circularly Polarised Light. RSC Adv. 2013, 3, 5213-5219. [CrossRef]

55. Fujiki, M.; Donguri, Y.; Zhao, Y.; Nakao, A.; Suzuki, N.; Yoshida, K.; Zhang, W. Photon Magic: Chiroptical Polarisation, Depolarisation, Inversion, Retention and Switching of Non-Photochromic Light-Emitting Polymers in Optofluidic Medium. Polym. Chem. 2015, 6, 1627-1638. [CrossRef]

56. Wang, L.; Yin, L.; Zhang, W.; Zhu, X.; Fujiki, M. Circularly Polarized Light with Sense and Wavelengths To Regulate Azobenzene Supramolecular Chirality in Optofluidic Medium. J. Am. Chem. Soc. 2017, 139, 13218-13226. [CrossRef]

57. Auzinsh, M.; Blushs, K.; Ferber, R.; Gahbauer, F.; Jarmola, A.; Tamanis, M. Electric Field Induced Symmetry Breaking of Angular Momentum Distribution in Atoms. Phys. Rev. Lett. 2006, 97, 043002. [CrossRef] [PubMed]

58. Bhattacharyya, K.; Surendran, A.; Chowdhury, C.; Datta, A. Steric and Electric Field Driven Distortions in Aromatic Molecules: Spontaneous and Non-Spontaneous Symmetry Breaking. Phys. Chem. Chem. Phys. 2016, 18, 31160-31167. [CrossRef] [PubMed]

59. Tielens, A.G.G.M. Interstellar Polycyclic Aromatic Hydrocarbon Molecules. Annu. Rev. Astron. Astrophys. 2008, 46, 289-337. [CrossRef]

60. Avalos, M.; Babiano, R.; Cintas, P.; Jiménez, J.L.; Palacios, J.C.; Barron, L.D. Absolute Asymmetric Synthesis under Physical Fields: Facts and Fictions. Chem. Rev. 1998, 98, 2391-2404. [CrossRef] [PubMed]

61. Zadel, G.; Eisenbrdun, C.; Wolff, G.-J.; Breitmaier, E. Enantioselective Reactions in a Static Magnetic Field-A False Alarm! Angew. Chem. Int. Ed. Engl. 1994, 33, 454-456. [CrossRef]

62. Kaupp, G.; Marquardt, T. Absolute Asymmetric Synthesis Solely under the Influence of a Static Homogeneous Magnetic Field? Angew. Chem. Int. Ed. 1994, 33, 1459-1461. [CrossRef]

63. Feringa, B.L.; Kellogg, R.M.; Hulst, R.; Zondervan, C.; Kruizinga, W.H. Attempts to Carry Out Enantioselective Reactions in a Static Magnetic Field. Angew. Chem. Int. Ed. 1994, 33, 1458-1459. [CrossRef]

64. Gölitz, P. Enantioselective Reactions in a Static Magnetic Field-False Alarm! Angew. Chem. Int. Ed. 1994, 33, 1457. [CrossRef]

65. Barron, L.D. Can A Magnetic Field Induce Absolute Asymmetric Synthesis? Science 1994, 266, 1491-1492. [CrossRef]

66. Naaman, R.; Wadeck, D.H. Chiral-Induced Spin Selectivity Effect. J. Phys. Chem. Lett. 2012, 3, 2178-2187. [CrossRef]

67. Banerjee-Ghosh, K.; Dor, O.B.; Tassinari, F.; Capua, E.; Yochelis, S.; Capua, A.; Yang, S.-H.; Parkin, S.S.P.; Sarkar, S.; Kronik, L.; et al. Separation of Enantiomers by Their Enantiospecific Interaction with Achiral Magnetic Substrates. Science 2018, 360, 1331-1334. [CrossRef] [PubMed]

68. Tassinari, F.; Steidel, J.; Paltiel, S.; Fontanesi, C.; Lahav, M.; Paltiel, Y.; Naaman, R. Enantioseparation by Crystallization Using Magnetic Substrates. Chem. Sci. 2019, 10, 5246-5250. [CrossRef] [PubMed]

69. Lee, T.D.; Yang, C.N. Question of Parity Conservation in Weak Interactions. Phys. Rev. 1956, 104, 254-258. [CrossRef]

70. Wu, C.S.; Ambler, E.; Hayword, R.W.; Hoppes, D.D.; Hudson, R.P. Experimental Test of Parity Conservation on Beta Decay. Phys. Rev. 1957, 105, 1413-1415. [CrossRef]

71. Ambler, E.; Hayward, R.W.; Hoppes, D.D.; Hudson, R.P.; Wu, C.S. Further Experiments on $\beta$ Decay of Polarized Nuclei. Phys. Rev. 1957, 106, 1361-1363. [CrossRef]

72. Postma, H.; Huiskamp, W.J.; Miedema, A.R.; Steenland, M.J.; Tolhoek, H.A.; Gorter, C.J. Asymmetry of the Positron Emission by Polarized ${ }^{58}$ Co Nuclei. Physica 1957, 23, 259-260. [CrossRef] 
73. Rodberg, L.S.; Weisskopf, V.F. Fall of Parity-Recent Discoveries Related to Symmetry of Laws of Nature. Science 1957, 125, 627-633. [CrossRef]

74. Goldhaber, M.; Grodzins, L.; Sunyar, A.W. Helicity of Neutrinos. Phys. Rev. 1958, 109, 1015-1017. [CrossRef]

75. Wróblewski, A.K. The Downfall of Parity-The Revolution That Happened Fifty Years Ago. Acta. Phys. Pol. B 2008, 39, 251-264.

76. Wu, C.S. The Discovery of the Parity Violation in Weak Interactions and Its Recent Developments. Lect. Notes Phys. 2008, 746, 43-49.

77. Stevenson, C.D.; Davis, J.P. Magnetars and Magnetic Separation of Chiral Radicals in Interstellar Space: Homochirality. J. Phys. Chem. A 2019, 123, 9587-9593. [CrossRef] [PubMed]

78. Kouveliotou, C.; Duncan, R.C.; Thompson, C. Magnetars. Sci. Am. 2003, 288, 34-41. [CrossRef] [PubMed]

79. Ohno, O.; Kaizu, Y.; Kobayashi, H. J-aggregate Formation of a Water-Soluble Porphyrin in Acidic Aqueous Media. J. Chem. Phys. 1993, 99, 4128-4139. [CrossRef]

80. Ribó, J.M.; Crusats, J.; Sagués, F.; Claret, J.; Rubires, R. Chiral Sign Induction by Vortices During the Formation of Mesophases in Stirred Solutions. Science 2001, 292, 2063-2066. [CrossRef]

81. Crusats, J.; El-Hachemi, Z.; Ribó, J.M. Hydrodynamic Effects on Chiral Induction. Chem. Soc. Rev. 2010, 39, 569-577. [CrossRef]

82. Okano, K.; Taguchi, M.; Fujiki, M.; Yamashita, T. Circularly Polarized Luminescence of Rhodamine B in a Supramolecular Chiral Medium Formed by a Vortex Flow. Angew. Chem. Int. Ed. 2011, 50, 12474-12477. [CrossRef]

83. Sun, J.; Li, Y.; Yan, F.; Liu, C.; Sang, Y.; Tian, F.; Feng, Q.; Duan, P.; Zhang, L.; Shi, X.; et al. Control over the Emerging Chirality in Supramolecular Gels and Solutions by Chiral Microvortices in Milliseconds. Nat. Commun. 2018, 9, 2599. [CrossRef]

84. Buckingham, A.D.; Stephens, P.J. Magnetic Optical Activity. Annu. Rev. Phys. Chem. 1966, 17, 399-432. [CrossRef]

85. Schatz, P.N.; McCaffery, A.J. Faraday effect. Q. Rev. Chem. Soc. 1969, 23, 552-584. [CrossRef]

86. Stephens, R.J. Magnetic Circular Dichroism. Annu. Rev. Phys. Chem. 1974, 25, 201-232. [CrossRef]

87. Kobayashi, N.; Muranaka, A.; Mack, J. Circular Dichroism and Magnetic Circular Dichroism Spectroscopy for Organic Chemists; RSC Publishing: Cambridge, UK, 2012.

88. Riehl, J.P.; Richardson, F.S. General Theory of Circularly Polarized Emission and Magnetic Circularly Polarized Emission from Molecular Systems. J. Chem. Phys. 1976, 65, 1011-1021. [CrossRef]

89. Wu, T.; Kapitán, J.; Andrushchenko, V.; Bouř, P. Identification of Lanthanide (III) Luminophores in Magnetic Circularly Polarized Luminescence Using Raman Optical Activity Instrumentation. Anal. Chem. 2017, 89, 5043-5049. [CrossRef] [PubMed]

90. Nelson, H.D.; Hinterding, S.O.M.; Fainblat, R.; Creutz, S.E.; Li, X.; Gamelin, D.R. A Selective Cation Exchange Strategy for the Synthesis of Colloidal $\mathrm{Yb}^{3+}$-Doped Chalcogenide Nanocrystals with Strong Broadband Visible Absorption and Long-Lived Near-Infrared Emission. J. Am. Chem. Soc. 2017, 139, 6411-6421. [CrossRef] [PubMed]

91. Ivchenko, E.L. Magnetic Circular Polarization of Exciton Photoluminescence. Phys. Solid State 2018, 60, 1514-1526. [CrossRef]

92. Kaji, D.; Okada, H.; Hara, N.; Kondo, Y.; Suzuki, S.; Miyasaka, M.; Fujiki, M.; Imai, Y. Non-classically Controlled Sign in a 1.6 Tesla Magnetic Circularly Polarized Luminescence of Three Pyrenes in a Chloroform and a PMMA Film. Chem. Lett. 2020, 49, 674-676. [CrossRef]

93. Okada, H.; Hara, N.; Kaji, D.; Shizuma, M.; Fujiki, M.; Imai, Y. Excimer-Origin CPL vs. Monomer-Origin Magnetic CPL in Photo-Excited Chiral Binaphthyl-Ester-Pyrenes: Critical Role of Ester Direction. Phys. Chem. Chem. Phys. 2020, 22, 13862-13866. [CrossRef] [PubMed]

94. Yoshikawa, H.; Nakajima, G.; Mimura, Y.; Kimoto, T.; Kondo, Y.; Suzuki, S.; Fujiki, M.; Imai, Y. Mirror-Image Magnetic Circularly Polarized Luminescence (MCPL) from Optically Inactive Eu ${ }^{\mathrm{III}}$ and Tb ${ }^{\mathrm{III}}$ Tris( $\beta$-Diketonate). Dalton Trans. 2020, 49, $9588-9594$. [CrossRef]

95. Raman, C.V.; Krishna, K.S. A New Type of Secondary Radiation. Nature 1928, 121, 501-502. [CrossRef]

96. Rikken, G.L.J.A.; Raupach, E. Enantioselective Magnetochiral Photochemistry. Nature 2000, 405, 932-934. [CrossRef]

97. Sharma, A. Enantiomeric Excess by Magnetic Circular Dichroism in Archaean Atmosphere. Sci. Rep. 2017, 7, 13295. [CrossRef]

98. Vester, F.; Ulbricht, T.L.V. Asymmetry: The Non-Conservation of Parity and Optical Activity. Quart. Rev. 1959, $13,48-60$.

99. Vester, F.; Ulbricht, T.L.V. Attempts to Induce Optically Activity with Polarized $\beta$-Radiation. Tetrahedron 1962, $18,629-637$.

100. Bonner, W.A. Experimental Evidence for $\beta$-Decay as a Source of Chirality by Enantiomer Analysis. Orig. Life 1984, 14, 383-390. [CrossRef] [PubMed]

101. Gidley, D.W.; Rich, A.; Van House, J.; Zitzewitz, P.W. $\beta$ Decay and the Origins of Biological Chirality: Experimental Results. Nature 1982, 297, 639-643. [CrossRef]

102. Van House, J.; Rich, A.; Zitzewitz, P.W. Beta Decay and The Origin of Biological Chirality: New Experimental Results. Orig. Life 1984, 14, 413-420. [CrossRef]

103. Dreiling, J.; Gay, T. Chirally Sensitive Electron-Induced Molecular Breakup and the Vester-Ulbricht Hypothesis. Phys. Rev. Lett. 2014, 113, 118103. [CrossRef]

104. Electroweak Interaction. Available online: https:/ / en.wikipedia:wiki/Electroweak_interaction (accessed on 20 December 2020).

105. Janoschek, R. Theories on the origin of biomolecular Homochirality. In Chirality_From Weak Bosons to the $\alpha$-Helix; Janoschek, R., Ed.; Springer: Berlin, Germany, 1991; ISBN 978-3-642-76569-8.

106. Close, E.E. Parity Violation in Atoms? Nature 1976, 264, 505-506. [CrossRef]

107. Bucksbaum, P.H.; Commins, E.D.; Hunter, L.R. Observations of Parity Nonconservation in Atomic Thallium. Phys. Rev. D 1981, 24, 1134-1148. [CrossRef] 
108. Emmons, T.P.; Reeves, J.M.; Fortson, E.N. Parity-Nonconserving Optical Rotation in Atomic Lead. Phys. Rev. Lett. 1983, 51, 2089-2091. [CrossRef]

109. Bouchiat, M.-A.; Pottier, L. Optical Experiments and Weak Interactions. Science 1986, 234, 1203-1210. [CrossRef]

110. World Scientific. Parity Violation in Atoms and Polarized Electron Scattering; Bernard, F., Bouchiat, M.-A., Eds.; World Scientific: Singapore, 1999; ISBN 9810237316.

111. Okun, L.B. Mirror Particles and Mirror Matter: 50 years of Speculation and Search. Phys. Usp. 2007, 50, 380-389. [CrossRef]

112. Tsigutkin, K.; Dounas-Frazer, D.; Family, A.; Stalnaker, J.E.; Yashchuk, V.V.; Budker, D. Observation of a Large Atomic Parity Violation Effect in Ytterbium. Phys. Rev. Lett. 2009, 103, 071601. [CrossRef]

113. Mason, A.; Tranter, G.E. Energy Inequivalence of Peptide Enantiomers from Parity Non-Conservation. J. Chem. Soc. Chem. Commun. 1983, 117-119. [CrossRef]

114. Quack, M. Structure and Dynamics of Chiral Molecules. Angew. Chem. Int. Ed. 1989, 28, 571-586. [CrossRef]

115. Quack, M.; Stohner, J.; Willeke, M. High-Resolution Spectroscopic Studies and Theory of Parity Violation in Chiral Molecules. Annu. Rev. Phys. Chem. 2008, 59, 741-769. [CrossRef]

116. Quack, M. How Important is Parity Violation for Molecular and Biomolecular Chirality? Angew. Chem. Int. Ed. 2002, 41, 4618-4630. [CrossRef]

117. Schwerdtfeger, P.; Gierlich, J.; Bollwein, T. Large Parity-Violation Effects in Heavy-Metal-Containing Chiral Compounds. Angew. Chem. Int. Ed. 2003, 42, 1293-1296. [CrossRef]

118. Darquié, B.; Stoeffler, C.; Shelkovnikov, A.; Daussy, C.; Amy-Klein, A.; Chardonnet, C.; Zrig, S.; Guy, L.; Crassous, J.; Soulard, P.; et al. Progress Toward the First Observation of Parity Violation in Chiral Molecules by High-Resolution Laser Spectroscopy. Chirality 2010, 22, 870-884. [CrossRef]

119. Berger, R. Molecular Parity Violation in Electronically Excited States. Phys. Chem. Chem. Phys. 2003, 5, 12-17. [CrossRef]

120. Fujiki, M.; Koe, J.R.; Mori, T.; Kimura, Y. Questions of Mirror Symmetry at the Photoexcited and Ground States of Non-Rigid Luminophores Raised by Circularly Polarized Luminescence and Circular Dichroism Spectroscopy: Part 1. Oligofluorenes, Oligophenylenes, Binaphthyls and Fused Aromatics. Molecules 2018, 23, 2606. [CrossRef]

121. Fujiki, M.; Koe, J.R.; Amazumi., S. Questions of Mirror Symmetry at the Photoexcited and Ground States of Non-Rigid Luminophores Raised by Circularly Polarized Luminescence and Circular Dichroism Spectroscopy. Part 2: Perylenes, BODIPYs, Molecular Scintillators, Coumarins, Rhodamine B, and DCM. Symmetry 2019, 11, 363. [CrossRef]

122. Panspermia. Available online: https://en.wikipedia.org/wiki/Panspermia\#cite_note-cometary_panspermia-3 (accessed on 2 November 2020).

123. Wickramasinghe, N.C. Search for Our Cosmic Ancestry; World Scientific: Singapore, 2014.

124. Kawaguchi, Y.; Yang, Y.; Kawashiri, N.; Shiraishi, K.; Takasu, M.; Narumi, I.; Satoh, K.; Hashimoto, H.; Nakagawa, K.; Tanigawa, Y.; et al. The Possible Interplanetary Transfer of Microbes: Assessing the Viability of Deinococcus spp. Under the ISS Environmental Conditions for Performing Exposure Experiments of Microbes in the Tanpopo Mission. Orig. Life. Evol. Biosph. 2013, 43, 411-428. [CrossRef] [PubMed]

125. Kawaguchi, Y.; Shibuya, M.; Kinoshita, I.; Yatabe, J.; Narumi, I.; Shibata, H.; Hayashi, R.; Fujiwara, D.; Murano, Y.; Hashimoto, H.; et al. DNA Damage and Survival Time Course of Deinococcal Cell Pellets During 3 Years of Exposure to Outer Space. Front. Microbiol. 2020, 11, 2050. [CrossRef] [PubMed]

126. Paganini, L.; Villanueva, G.L.; Roth, L.; Mandell, A.M.; Hurford, T.A.; Retherford, K.D.; Mumma, M.J. A Measurement of Water Vapour Amid a Largely Quiescent Environment on Europa. Nat. Astron. 2020, 4, 266-272. [CrossRef]

127. Goesmann, F.; Rosenbauer, H.; Bredehöft, J.H.; Cabane, M.; Ehrenfreund, P.; Gautier, T.; Giri, C.; Krüger, H.; Roy, L.L.; MacDermott, A.J.; et al. Organic Compounds on Comet 67P/Churyumov-Gerasimenko Revealed by COSAC Mass Spectrometry. Science 2015, 349, aab0689. [CrossRef]

128. Bibring, J.-P.; Langevin, Y.; Carter, J.; Eng, P.; Gondet, B.; Jorda, L.; Le Mouélic, S.; Mottola, S.; Pilorget, C.; Poulet, F.; et al. 67P/Churyumov-Gerasimenko Surface Properties as Derived from CIVA Panoramic Images. Science 2015, 349, aab0671. [CrossRef]

129. Iess, L.; Stevenson, D.J.; Parisi, M.; Hemingway, D.; Jacobson, R.A.; Lunine, J.I.; Nimmo, F.; Armstrong, J.W.; Asmar, S.W.; Ducci, M.; et al. The Gravity Field and Interior Structure of Enceladus. Science 2014, 344, 78-80. [CrossRef]

130. Lorenz, R.D.; Kirk, R.L.; Hayes, A.G.; Anderson, Y.Z.; Lunine, J.I.; Tokano, T.; Turtle, E.P.; Malaska, M.J.; Soderblom, J.M.; Lucas, A.; et al. A Radar Map of Titan Seas: Tidal Dissipation and Ocean Mixing through the Throat of Kraken. Icarus 2014, 237, 9-15. [CrossRef]

131. Pepe, F.; Lovis, C.; Ségransan, D.; Benz, W.; Bouchy, F.; Dumusque, X.; Mayor, M.; Queloz, D.; Santos, N.C.; Udry, S. The HARPS Search for Earth-like Planets in the Habitable Zone. Astron. Astrophys. 2011, 534, A58. [CrossRef]

132. Quintana, E.V.; Barclay, T.; Raymond, S.N.; Rowe, J.F.; Bolmont, E.; Caldwell, D.A.; Howell, S.B.; Kane, S.R.; Huber, D.; Crepp, J.R.; et al. An Earth-sized Planet in the Habitable Zone of a Cool Star. Science 2014, 344, 277-280. [CrossRef]

133. McGuire, B.A.; Carroll, P.B.; Loomis, R.A.; Finneran, I.A.; Jewell, P.R.; Remijan, A.J.; Blake, G.A. Discovery of the Interstellar Chiral Molecule Propylene Oxide $\left(\mathrm{CH}_{3} \mathrm{CHCH}_{2} \mathrm{O}\right)$. Science 2016, 352, 1449-1452. [CrossRef] [PubMed]

134. Furukawa, Y.; Chikaraishi, Y.; Ohkouchi, N.; Ogawa, N.O.; Gavin, D.P.; Dworkin, J.P.; Abe, C.; Nakamura, T. Extraterrestrial Ribose and Other Sugars in Primitive Meteorites. Proc. Natl. Acad. Sci. USA 2019, 116, 24440-24445. [CrossRef] [PubMed] 
135. Hayabusa-2 Project. Available online: http://www.hayabusa2.jaxa.jp/en/topics/20201209_capsulephotos/ (accessed on 9 December 2020).

136. Miller, S.L. Production of Amino Acids under Possible Primitive Earth Conditions. Science 1953, 117, 528-529. [CrossRef]

137. Miller, S.L.; Urey, H.C. Organic Compound Synthesis on the Primitive Earth. Science 1959, 130, 245-251. [CrossRef] [PubMed]

138. Bada, J.L. New Insights into Prebiotic Chemistry from Stanley Miller's Spark Discharge Experiments. Chem. Soc. Rev. 2013, 42, 2186-2196. [CrossRef]

139. Enoto, T.; Wada, Y.; Furuta, Y.; Nakazawa, K.; Yuasa, T.; Okuda, K.; Makishima, K.; Sato, M.; Sato, Y.; Nakano, T.; et al. Photonuclear Reactions Triggered by Lightning Discharge. Nature 2017, 551, 481-484. [CrossRef]

140. Rozanski, K.; Fröhlich, K. Radioactivity and Earth Sciences: Understanding the Natural Environment. IAEA Bull. 1996, 38, 9-15.

141. Araki, T.; Enomoto, S.; Furuno, K.; Gando, Y.; Ichimura, K.; Ikeda, H.; Inoue, K.; Kishimoto, Y.; Koga, M.; Koseki, Y.; et al. Experimental Investigation of Geologically Produced Antineutrinos with KamLAND. Nature 2005, 436, 499-503. [CrossRef]

142. Rosianna, I.; Nugraha, E.D.; Syaeful, H.; Putra, S.; Hosoda, M.; Akata, N.; Tokonami, S. Natural Radioactivity of Laterite and Volcanic Rock Sample for Radioactive Mineral Exploration in Mamuju, Indonesia. Geosciences 2020, 10, 376. [CrossRef]

143. Córdova, A.; Engqvist, M.; Ibrahem, I.; Casa, J.; Sundén, H. Plausible Origins of Homochirality in the Amino Acid Catalyzed Neogenesis of Carbohydrates. Chem. Commun. 2005, 2047-2049. [CrossRef]

144. Sato, I.; Urabe, H.; Ishiguro, S.; Shibata, T.; Soai, K. Amplification of Chirality from Extremely Low to Greater than $99.5 \%$ ee by Asymmetric Autocatalysis. Angew. Chem. Int. Ed. 2003, 42, 315-317. [CrossRef] [PubMed]

145. Haldane, J.B.S. An Exact Test for Randomness of Mating. New Biol. 1954, 16, $12-27$.

146. Lu, T.; Spruijt, E. Multiphase Complex Coacervate Droplets. J. Am. Chem. Soc. 2020, 142, 2905-2914. [CrossRef] [PubMed]

147. Favorskiy, I.; Vu, D.; Peytavit, E.; Arscott, S.; Paget, D.; Rowe, A.C.H. Circularly Polarized Luminescence Microscopy for the Imaging of Charge and Spin Diffusion in Semiconductors. Rev. Sci. Instrum. 2010, 81, 103902. [CrossRef] [PubMed]

148. Kaminsky, W.; Claborn, K.; Kahr, B. Polarimetric Imaging of Crystals. Chem. Soc. Rev. 2004, 33, 514-525. [CrossRef] [PubMed]

149. Narushima, T.; Okamoto, H. Circular Dichroism Microscopy Free from Commingling Linear Dichroism via Discretely Modulated Circular Polarization. Sci. Rep. 2016, 6, 35731. [CrossRef]

150. Le, K.Q.; Hashiyada, S.; Kondo, M.; Okamoto, H. Circularly Polarized Photoluminescence from Achiral Dye Molecules Induced by Plasmonic Two-Dimensional Chiral Nanostructures. J. Phys. Chem. C 2018, 122, 24924-24932. [CrossRef]

151. Claborn, K.; Puklin-Faucher, E.; Kurimoto, M.; Kaminsky, W.; Kahr, B. Circular Dichroism Imaging Microscopy: Application to Enantiomorphous Twinning in Biaxial Crystals of 1,8-Dihydroxyanthraquinone. J. Am. Chem. Soc. 2003, 125, 14825-14831. [CrossRef]

152. Narushima, T.; Okamoto, H. Circular Dichroism Nano-Imaging of Two-Dimensional Chiral Metal Nanostructures. Phys. Chem. Chem. Phys. 2013, 15, 13805-13809. [CrossRef]

153. Hauser, E.A. The History of Colloid Science: In Memory of Wolfgang Ostwald. J. Chem. Educ. 1955, 32, 2-9. [CrossRef]

154. Le Chatelier, H. Crystalloids Against Colloids in the Theory of Cements. Trans. Faraday Soc. 1919, 14, 8-11. [CrossRef]

155. Hermann Staudinger. Available online: https://en.wikipedia.org/wiki/Hermann_Staudinger (accessed on 11 December 2020).

156. Terayama, H. Method of colloid titration (a new titration between polymer ions). J. Polym. Sci. 1952, 8, 243-253. [CrossRef]

157. Kondepudi, D.K.; Kaufman, R.J.; Singh, N. Chiral Symmetry Breaking Crystallization. Science 1990, 250, 975-976. [CrossRef] [PubMed]

158. Martin, B.; Tharrington, A.; Wu, X.-I. Chiral Symmetry Breaking in Crystal Growth: Is Hydrodynamic Convection Relevant? Phys. Rev. Lett. 1996, 77, 2826-2829. [CrossRef] [PubMed]

159. Viedma, C. Chiral Symmetry Breaking During Crystallization: Complete Chiral Purity Induced by Nonlinear Autocatalysis and Recycling. Phys. Rev. Lett. 2005, 94, 065504. [CrossRef] [PubMed]

160. Viedma, C.; Ortiz, J.E.; Torres, T.D.; Izumi, T.; Blackmond, D.G. Evolution of Solid Phase Homochirality for a Proteinogenic Amino Acid. J. Am. Chem. Soc. 2008, 130, 15274-15275. [CrossRef]

161. Noorduin, W.L.; Vlieg, E.; Kellogg, R.M.; Kaptein, B. From Ostwald Ripening to Single Chirality. Angew. Chem. Int. Ed. 2009, 48, 9600-9606. [CrossRef]

162. Viedma, C. Selective Chiral Symmetry Breaking during Crystallization: Parity Violation or Cryptochiral Environment in Control? Cryst. Growth Des. 2007, 7, 553-556. [CrossRef]

163. Qian, S.X.; Snow, J.B.; Tzeng, H.M.; Chang, R.K. Lasing Droplets: Highlighting the Liquid-Air Interface by Laser Emission. Science 1986, 231, 486-488. [CrossRef]

164. Psaltis, D.; Quack, S.R.; Yang, C. Developing Optofluidic Technology through the Fusion of Microfluidics and Optics. Nature 2006, 442, 381-386. [CrossRef]

165. Fan, X.; White, I.M. Optofluidic Microsystems for Chemical and Biological Analysis. Nat. Photonics 2011, 5, 591-597. [CrossRef] [PubMed]

166. Schmidt, A.H.; Hawkins, A.R. The Photonic Integration of Non-Solid Media using Optofluidics. Nat. Photon. 2011, 5, 598-604. [CrossRef]

167. Fainman, Y.; Lee, L.P.; Psaltis, D. Optofluidics; Yang, C., Ed.; McGraw-Hill: New York, NY, USA, 2010.

168. Tang, S.K.Y.; Li, Z.; Abate, A.R.; Agresti, J.J.; Weitz, D.A.; Psaltis, D.; Whitesides, G.M. A Multi-Color Fast-Switching Microfluidic Droplet Dye Laser. Lab Chip 2009, 9, 2767-2771. [CrossRef] [PubMed] 
169. Schäfer, J.; Mondia, J.P.; Sharma, R.; Lu, Z.H.; Susha, A.S.; Rogach, A.L.; Wang, L.J. Quantum Dot Microdrop Laser. Nano Lett. 2008, 8, 1709-1712. [CrossRef]

170. Domachuk, P.; Littler, I.C.M.; Cronin-Golomb, M.; Eggletona, B.J. Compact Resonant Integrated Microfluidic Refractometer. Appl. Phys. Lett. 2006, 88, 093513. [CrossRef]

171. Steinberg, I.Z. Circular Polarization of Luminescence: Biochemical and Biophysical Applications. Annu. Rev. Biophys. Bioeng. 1978, 7, 113-137. [CrossRef]

172. Barzda, V.; Musthrdy, L.; Garab, G. Size Dependency of Circular Dichroism in Macrocolloids of Photosynthetic Pigment-Protein Complexes. Biochemistry 1994, 33, 10837-10841. [CrossRef]

173. Barzda, V.; Istokovics, A.; Simidjiev, I.; Garab, G. Structural Flexibility of Chiral Macrocolloids of Light-Harvesting Chlorophyll a/b Pigment-Protein Complexes. Light-Induced Reversible Structural Changes Associated with Energy Dissipation. Biochemistry 1996, 35, 8981-8985. [CrossRef]

174. Gussakovsky, E.E.; Shahak, Y.; van Amerongen, H.; Barzda, V. Circularly Polarized Chlorophyll Luminescence Reflects the Macro-Organization of Grana in Pea Chloroplasts. Photosynth. Res. 2000, 65, 83-92. [CrossRef]

175. Dobson, C.M.; Ellison, G.B.; Tuck, A.F.; Vaida, V. Atmospheric aerosols as prebiotic chemical reactors. Proc. Natl. Acad. Sci. USA 2000, 97, 11864-11868. [CrossRef]

176. Nam, I.; Lee, J.K.; Nam, H.G.; Zare, R.N. Abiotic Production of Sugar Phosphates and Uridine Ribonucleoside in Aqueous Microdroplets. Proc. Natl. Acad. Sci. USA 2017, 114, 12396-12400. [CrossRef] [PubMed]

177. Nam, I.; Nam, H.G.; Zare, R.N. Abiotic Synthesis of Purine and Pyrimidine Ribonucleosides in Aqueous Microdroplets. Proc. Natl. Acad. Sci. USA 2018, 115, 36-40. [CrossRef] [PubMed]

178. Lee, J.K.; Banerjee, S.; Nam, H.G.; Zare, R.N. Acceleration of Reaction in Charged Microdroplets. Q. Rev. Biophys. 2015, 48, 437-444. [CrossRef]

179. Mortensen, D.N.; Williams, E.R. Microsecond and Nanosecond Polyproline II Helix Formation in Aqueous Nanodrops Measured by Mass Spectrometry. Chem. Commun. 2016, 52, 12218-12221. [CrossRef] [PubMed]

180. Guardingo, M.; Busqué, F.; Ruiz-Molina, D. Reactions in Ultra-Small Droplets by Tip-Assisted Chemistry. Chem. Commun. 2016, 52, 11617-11626. [CrossRef] [PubMed]

181. Lee, J.K.; Samanta, D.; Nam, H.G.; Zare, R.N. Micrometer-Sized Water Droplets Induce Spontaneous Reduction. J. Am. Chem. Soc. 2019, 141, 10585-10589. [CrossRef] [PubMed]

182. Zhou, Z.; Yan, X.; Lai, Y.H.; Zare, R.N. Fluorescence Polarization Anisotropy in Microdroplets. J. Phys. Chem. Lett. 2018, 9 , 2928-2932. [CrossRef]

183. Kageyama, Y. Robust Dynamics of Synthetic Molecular Systems as a Consequence of Broken Symmetry. Symmetry 2020, $12,1688$. [CrossRef]

184. Eliel, E.L.; Wilen, S.H. Chiroptical properties. In Stereochemistry of Organic Compounds; John Wiley \& Sons, Inc.: New York, NY, USA, 1994; pp. 991-1118.

185. Fujiki, M.; Wang, L.; Ogata, N.; Asanoma, F.; Okubo, A.; Okazaki, S.; Kamite, H.; Jalilah, A.J. Chirogenesis and Pfeiffer Effect in Optically Inactive $\mathrm{Eu}^{\mathrm{III}}$ and $\mathrm{Tb}^{\mathrm{III}}$ Tris ( $\beta$-diketonate) Upon Intermolecular Chirality Transfer From Poly-and Monosaccharide Alkyl Esters and $\alpha$-Pinene: Emerging Circularly Polarized Luminescence (CPL) and Circular Dichroism (CD). Front. Chem. 2020, 8,685 .

186. Albano, G.; Pescitelli, G.; Di Bari, L. Chiroptical Properties in Thin Films of $\pi$-Conjugated Systems. Chem. Rev. 2020, 120, 10145-10243. [CrossRef]

187. Mei, J.; Leung, N.L.; Kwok, R.T.; Lam, J.W.; Tang, B.Z. Aggregation-Induced Emission: Together We Shine, United We Soar! Chem. Rev. 2015, 115, 11718-11940. [CrossRef] [PubMed]

188. Song, F.; Zhao, Z.; Liu, Z.; Lam, J.W.; Tang, B.Z. Circularly Polarized Luminescence from AIEgens. J. Mater. Chem. C 2020, 8, 3284-3301. [CrossRef]

189. Luo, J.; Xie, Z.; Lam, J.W.Y.; Cheng, L.; Chen, H.; Qiu, C.; Kwok, H.S.; Zhan, X.; Liu, Y.; Zhu, D.; et al. Aggregation-Induced Emission of 1-Methyl-1,2,3,4,5-Pentaphenylsilole. Chem. Commun. 2001, 1740-1741. [CrossRef] [PubMed]

190. Chen, J.; Law, C.C.W.; Lam, J.W.Y.; Dong, Y.; Lo, S.M.F.; Williams, I.D.; Zhu, D.; Tang, B.Z. Synthesis, Light Emission, Nanoaggregation, and Restricted Intramolecular Rotation of 1,1-Substituted 2,3,4,5-Tetraphenylsiloles. Chem. Mater. 2003, 15, 1535-1546. [CrossRef]

191. Liu, J.; Su, H.; Meng, L.; Zhao, Y.; Deng, C.; Ng, J.C.Y.; Lu, P.; Faisal, M.; Lam, J.W.Y.; Huang, X.; et al. What Makes Efficient Circularly Polarised Luminescence in the Condensed Phase: Aggregation-induced Circular Dichroism and Light emission. Chem. Sci. 2012, 3, 2737-2747. [CrossRef]

192. Li, H.; Zheng, X.; Su, H.; Lam, J.W.Y.; Wong, K.S.; Xue, S.; Huang, X.; Huang, X.; Li, B.S.; Tang, B.Z. Synthesis, Optical Properties, and Helical Self-Assembly of a Bivaline-Containing Tetraphenylethene. Sci. Rep. 2016, 6, 19277.

193. Ghosh, A.; Fischer, P. Chiral Molecules Split Light: Reflection and Refraction in a Chiral Liquid. Phys. Rev. Lett. 2006, 97, 173002. [CrossRef]

194. Silverman, M.P.; Badoz, J.; Briat, B. Chiral Reflection from a Naturally Optically Active Medium. Opt. Lett. 1992, 17, 886-888. [CrossRef]

195. Pedersen, J.; Mortensen, N.A. Enhanced Circular Dichroism via Slow Light in Dispersive Structured Media. Appl. Phys. Lett. 2007, 91, 213501. [CrossRef] 
196. Mortensen, N.A.; Xiao, S. Slow-Light Enhancement of Beer-Lambert-Bouguer Absorption. Appl. Phys. Lett. 2007, 90, 141108. [CrossRef]

197. Uemura, N.; Toyoda, S.; Shimizu, W.; Yoshida, Y.; Mino, T.; Sakamoto, M. Absolute Asymmetric Synthesis Involving Chiral Symmetry Breaking in Diels-Alder Reaction. Symmetry 2020, 12, 910. [CrossRef]

198. Kawagoe, K.; Fujiki, M.; Nakano, Y. Limonene Magic: Noncovalent Molecular Chirality Transfer Leading to Ambidextrous Circularly Polarised Luminescent $\pi$-Conjugated Polymers. New J. Chem. 2010, 34, 637-647. [CrossRef]

199. Nakano, Y.; Fujiki, M. Circularly Polarized Light Enhancement by Helical Polysilane Aggregates Suspension in Organic Optofluids. Macromolecules 2011, 48, 7511-7919. [CrossRef]

200. Nakano, Y.; Ichiyanagi, F.; Naito, M.; Yang, Y.-G.; Fujiki, M. Chiroptical Generation and Inversion during the Mirror-SymmetryBreaking Aggregation of Dialkylpolysilanes due to Limonene Chirality. Chem. Commun. 2012, 48, 6636-6638. [CrossRef] [PubMed]

201. Nakano, Y.; Liu, Y.; Fujiki, M. Ambidextrous Circular Dichroism and Circularly Polarised Luminescence from Poly (9,9-di- $n$ decylfluorene) by Terpene Chirality Transfer. Polym. Chem. 2010, 1, 460-469. [CrossRef]

202. Zhang, W.; Yoshida, K.; Fujiki, M.; Zhu, X. Unpolarized-Light-Driven Amplified Chiroptical Modulation Between Chiral Aggregation and Achiral Disaggregation of an Azobenzene-alt-Fluorene Copolymer in Limonene. Macromolecules 2011, 44, 5105-5111. [CrossRef]

203. Fujiki, M.; Jalilah, A.J.; Suzuki, N.; Taguchi, M.; Zhang, W.; Abdellatif, M.M.; Nomura, K. Chiral Optofluidics: Gigantic Circularly Polarized Light Enhancement of all-trans-Poly (9,9-di-n-octylfluorene-2,7-vinylene) during Mirror-Symmetry-Breaking Aggregation by Optically Tuning Fluidic Media. RSC Adv. 2012, 2, 6663-6671. [CrossRef]

204. Fujiki, M.; Kawagoe, Y.; Nakano, Y.; Nakao, A. Mirror-Symmetry-Breaking in Poly [(9,9-di- $n$-octylfluorenyl-2,7-diyl)-alt-biphenyl] (PF8P2) is Susceptible to Terpene Chirality, Achiral Solvents, and Mechanical Stirring. Molecules 2013, 18, 7035-7057. [CrossRef]

205. Wang, L.; Suzuki, N.; Liu, J.; Matsuda, T.; Rahim, N.A.A.; Zhang, W.; Fujiki, M.; Zhang, Z.; Zhou, N.; Zhu, X. Limonene Induced Chiroptical Generation and Inversion during Aggregation of Achiral Polyfluorene Analogs: Structure-Dependence and Mechanism. Polym. Chem. 2014, 5, 5920-5927. [CrossRef]

206. Liu, J.; Zhang, J.; Zhang, S.; Suzuki, N.; Fujiki, M.; Wang, L.; Li, L.; Zhang, W.; Zhou, N.; Zhu, X. Chiroptical Generation and Amplification of Hyperbranched $\pi$-Conjugated Polymers in Aggregation States Driven by Limonene Chirality. Polym. Chem. 2014, 5, 784-791. [CrossRef]

207. Fujiki, M.; Yoshida, K.; Suzuki, N.; Rahim, N.A.A.; Jalil, J.A. Tempo-Spatial Chirogenesis. Limonene-Induced Mirror Symmetry Breaking of Si-Si Bond Polymers during Aggregation in Chiral Fluidic Media. J. Photochem. Photobiol. A 2016, 331, 120-129. [CrossRef]

208. Rahim, N.A.A.; Fujiki, M. Aggregation-induced Scaffolding: Photoscissable Helical Polysilane Generates Circularly Polarized Luminescent Polyfluorene. Polym. Chem. 2016, 7, 4618-4629. [CrossRef]

209. Duong, S.T.; Fujiki, M. The Origin of Bisignate Circularly Polarized Luminescence (CPL) Spectra from Chiral Polymer Aggregates and Molecular Camphor: Anti-Kasha's Rule Revealed by CPL Excitation (CPLE) Spectra. Polym. Chem. 2017, 8, 4673-4679. [CrossRef]

210. Fujiki, M.; Yoshimoto, S. Time-evolved, Far-red, Circularly Polarised Luminescent Polymer Aggregates Endowed with Sacrificial Helical Si-Si Bond Polymers. Mater. Chem. Front. 2017, 1, 1773-1785. [CrossRef]

211. Chen, H.; Yin, L.; Liu, M.; Wang, L.; Fujiki, M.; Zhang, W.; Zhu, X. Aggregation-Induced Chiroptical Generation and Photoinduced Switching of Achiral Azobenzene-alt-Fluorene Copolymer Endowed with Left-and Right-Handed Helical Poly-silanes. RSC Adv. 2019, 9, 4849-4856. [CrossRef]

212. Fujiki, M. Aggregation-Induced Chirogenesis of Luminescent Polymers. In Aggregation-Induced Emission: Materials and Applications; Fujiki, M., Liu, B., Tang, B.Z., Eds.; American Chemical Society: Columbus, OH, USA, 2016; Volume 2, pp. 63-92.

213. Beth, R.A. Mechanical Detection and Measurement of the Angular Momentum of Light. Phys. Rev. 1936, 50, 115-125. [CrossRef]

214. Saha, M.N.; Bhargava, Y. The Spin of the Photon. Nature 1931, 128, 870. [CrossRef]

215. Simpson, N.B.; Dholakia, K.; Allen, L.; Padgett, M.J. Mechanical Equivalence of Spin and Orbital Angular Momentum of Light: An Optical Spanner. Opt. Lett. 1997, 22, 52-54. [CrossRef]

216. Turro, N.J. Modern Molecular Photochemistry; University Science Books: Sausalito, CA, USA, 1991 ; ISBN 0935702717.

217. Calvert, J.G.; Pitts, J.N. Photochemistry; John Wiley \& Sons: Hoboken, NJ, USA, 1973; ISBN 0471130907.

218. Franck-Condon-Principle. Available online: https://en.wikipedia.org/wiki/Franck--Condon_principle (accessed on 23 December 2020).

219. Nakashima, H.; Fujiki, M.; Koe, J.R.; Motonaga, M. Solvent and Temperature Effects on the Chiral Aggregation of Poly(alkylarylsilane) s Bearing Remote Chiral Groups. J. Am. Chem. Soc. 2001, 123, 1963-1969. [CrossRef]

220. Terao, K.; Mori, Y.; Dobashi, T.; Sato, T.; Teramoto, A.; Fujiki, M. Solvent and Temperature Effects on the Chiral Aggregation of Optically Active Poly (dialkylsilane)s Confined in Microcapsules. Langmuir 2004, 20, 306-308. [CrossRef]

221. Fujiki, M. Ideal Exciton Spectra in Single- and Double-Screw-Sense Helical Polysilanes. J. Am. Chem. Soc. 1994, 116, 6017-6018. [CrossRef]

222. Fujiki, M. Optically Active Polysilylenes: State-Of-The-Art Chiroptical Polymers. Macromol. Rapid Commun. 2001, 22, 539-563. [CrossRef] 
223. Fujiki, M.; Koe, J.R.; Terao, K.; Sato, T.; Teramoto, A.; Watanabe, J. Optically Active Polysilanes. Ten Years of Progress and New Polymer Twist for Nanoscience and Nanotechnology. Polym. J. 2003, 35, 297-344. [CrossRef]

224. Eliel, E.L.; Wilen, S.H.; Mander, L.N. The CD magnitude is normalized using the dimensionless Kuhn's anisotropy factor in the ground state, defined as $g_{\mathrm{CD}}=\Delta \varepsilon / \varepsilon=\left(A b_{\mathrm{SL}}-A b s_{\mathrm{R}}\right) /\left[\left(A b_{\mathrm{SL}}+A b s_{\mathrm{R}}\right) / 2\right]$ at extremum. In Stereochemistry of Organic Compounds; John Wiley \& Sons, Inc.: New York, NY, USA, 1994.

225. Eliel, E.L.; Wilen, S.H.; Mander, L.N. The CPL magnitude is normalized by Kuhn's anisotropy in the photoexcited state. $g_{\mathrm{CPL}}=(\mathrm{IL}$ $-I R) /[(I L+I R) / 2]$ at extremum, where IL and IR denote the emission intensities of the left- and right-CP light under excitation of unpolarized light, re-spectively. John Wiley \& Sons, Inc.: New York, NY, USA, 1994.

226. Nakashima, H.; Koe, J.R.; Torimitsu, K.; Fujiki, M. Transfer and Amplification of Chiral Molecular Information to Poly-silylene Aggregates. J. Am. Chem. Soc. 2001, 123, 4847-4848. [CrossRef] [PubMed]

227. Righini, G.C.; Dumeige, Y.; Féron, P.; Ferrari, M.; Conti, G.N.; Ristic, D.; Soria, S. Whispering Gallery Mode Microresonators: Fundamentals and Applications. Riv. Nuovo Cimento 2011, 34, 435-488.

228. Symes, R.; Sayer, R.M.; Reid, J.P. Cavity Enhanced Droplet Spectroscopy: Principles, Perspectives and Prospects. Phys. Chem. Chem. Phys. 2004, 6, 474-487. [CrossRef]

229. Li, Z.; Psaltis, D. Optofluidic Dye Lasers. Microfluid Nanofluid. 2008, 4, 145-158. [CrossRef]

230. Sun, Y.; Shopova, S.I.; Wu, C.S.; Arnold, S.; Fan, X. Bioinspired Optofluidic FRET Lasers via DNA Scaffolds. Proc. Nat. Acad. Sci. USA 2010, 107, 16039-16042. [CrossRef]

231. He, C.; Yang, G.; Kuai, Y.; Shan, S.; Yang, L.; Hu, J.; Zhang, D.; Zhang, Q.; Zou, G. Dissymmetry Enhancement in Enantioselective Synthesis of Helical Polydiacetylene by Application of Superchiral Light. Nat. Commun. 2018, 9, 5117. [CrossRef]

232. Yang, G.; Zhang, S.; Hu, J.; Fujiki, M.; Zou, G. The Chirality Induction and Modulation of Polymers by Circularly Polarized Light. Symmetry 2019, 11, 474. [CrossRef]

233. Kim, J.Y.; Yeom, J.; Zhao, G.; Calcaterra, H.; Munn, J.; Zhang, P.; Kotov, N. Assembly of Gold Nanoparticles into Chiral Superstructures Driven by Circularly Polarized Light. J. Am. Chem. Soc. 2019, 141, 11739-11744. [CrossRef] [PubMed]

234. Asano, T.; Okada, T.; Shinkai, S.; Shigematsu, K.; Kusano, Y.; Manabe, O. Temperature and Pressure Dependences of Thermal Cis-to-Trans Isomerization of Azobenzenes which Evidence an Inversion Mechanism. J. Am. Chem. Soc. 1981, 103, 5161-5165. [CrossRef]

235. Klimov, V.V.; Zabkov, I.V.; Pavlov, A.; Guzatov, D.V. Eigen Oscillations of a Chiral Sphere and Their Influence on Radiation of Chiral Molecules. Opt. Exp. 2014, 22, 18564-18578. [CrossRef] [PubMed]

236. Ureña, F.P.; Moreno, J.R.A.; González, J.J.L. Conformational Study of (R)-(+)-Limonene in the Liquid Phase using Vibrational Spectroscopy (IR, Raman, and VCD) and DFT Calculations. Tetrahedron Asymmetry 2009, 20, 89-97. [CrossRef]

237. Reinscheid, F.; Reinscheid, U.M. Stereochemical Analysis of (+)-Limonene using Theoretical and Experimental NMR and Chiroptical Data. J. Mol. Struct. 2016, 1106, 141-153. [CrossRef]

238. Gierschner, J.; Behera, S.K.; Park, S.Y. Dual Emission: Classes, Mechanisms and Conditions. Angew. Chem. Int. Ed. 2020. [CrossRef]

239. Röhrs, M.; Escudero, D. Multiple Anti-Kasha Emissions in Transition-Metal Complexes. J. Phys. Chem. Lett. 2019, 10, 5798-5804. [CrossRef]

240. Del Valle, J.C.; Catalán, J. Kasha's rule: A reappraisal. Phys. Chem. Chem. Phys. 2019, 21, 10061-10069. [CrossRef]

241. Demchenko, A.P.; Tomin, V.I.; Chou, P.T. Breaking the Kasha Rule for More Efficient Photochemistry. Chem. Rev. 2017, 117, 13353-13381. [CrossRef]

242. Kunitake, T.; Okahata, Y. A Totally Synthetic Bilayer Membrane. J. Am. Chem. Soc. 1977, 99, 3860-3861. [CrossRef]

243. Fujiki, M. Studies on Hydride Abstraction Reaction by Trityl Salts and Synthesis/Polymerization of Dialkylammonium Acrylate. Master's Thesis, Kyushu University, Fukuoka, Japan, 1978. (In Japanese).

244. Krishnamurthy, R.; Hud, N.V. Introduction: Chemical Evolution and the Origins of Life. Chem. Rev. 2020, 120, 4613-4615. [CrossRef] [PubMed]

245. Sandford, S.A.; Nuevo, M.; Bera, P.P.; Lee, T.J. Prebiotic Astrochemistry and the Formation of Molecules of Astrobiological Interest in Interstellar Clouds and Protostellar Disks. Chem. Rev. 2020, 120, 4616-4659. [CrossRef] [PubMed]

246. Glavin, D.P.; Burton, A.S.; Elsila, J.E.; Aponte, J.C.; Dworkin, J.P. The Search for Chiral Asymmetry as a Potential Biosignature in our Solar System. Chem. Rev. 2020, 120, 4660-4689. [CrossRef] [PubMed]

247. Ribó, J.M. Chirality: The Backbone of Chemistry as a Natural Science. Symmetry 2020, 12, 1982. [CrossRef]

248. Yashima, E.; Ousaka, N.; Taura, D.; Shimomura, K.; Ikai, T.; Katsuhiro Maeda, K. Supramolecular Helical Systems: Helical Assemblies of Small Molecules, Foldamers, and Polymers with Chiral Amplification and Their Functions. Chem. Rev. 2016, 116, 3752-13990. [CrossRef]

249. Borovkov, V.V.; Hembury, G.A.; Inoue, Y. Origin, Control, and Application of Supramolecular Chirogenesis in Bisporphyrin-Based Systems. Acc. Chem. Res. 2004, 37, 449-459. [CrossRef]

250. Plutschack, M.B.; Pieber, B.; Gilmore, K.; Seeberger, P.H. The Hitchhiker's Guide to Flow Chemistry II. Chem. Rev. 2017, 117, 11796-11893. [CrossRef] 
251. Brill, Z.G.; Condakes, M.L.; Ting, C.P.; Maimone, T.J. Navigating the Chiral Pool in the Total Synthesis of Complex Terpene Natural Products. Chem. Rev. 2017, 117, 11753-11795. [CrossRef]

252. Puneet, P.; Fujiki, M.; Nandan, B. Circularly Polarized Luminescent Polymers: Emerging Materials for Photophysical Applications. In Reactive and Functional Polymers; Gutierrez, T., Ed.; Springer: Cham, Switzerland, 2020; Volume 3, pp. 117-139. 